\title{
Erythropoietin-mediated neuroprotection in insects
}

\author{
DISSERTATION \\ for the award of the degree \\ "Doctor rerum naturalium" \\ Division of Mathematics and Natural Sciences \\ of the Georg-August-Universität Göttingen \\ within the doctoral programme Biology \\ at the Georg-August University School of Science (GAUSS)
}

submitted by

Nataša Miljuš

from Belgrade, Serbia

Göttingen, 2016 


\section{Thesis Committee Members}

\section{Prof. Dr. Ralf Heinrich (supervisor and first reviewer)}

Georg-August-Universität Göttingen, Faculty of Biology/JFB-Institute for Zoology and Anthropology, Department of Cellular Neurobiology

\section{Prof. Dr. Dr. Hannelore Ehrenreich (second reviewer)}

Max Planck Institute for Experimental Medicine, Göttingen, Clinical Neuroscience

Other Members of the Examination Board

\section{Prof. Dr. Gregor Bucher}

Georg-August-Universität Göttingen, Faculty of Biology/JFB-Institute for Zoology and Anthropology, Department of Developmental Biology

\section{Prof. Dr. André Fiala}

Georg-August-Universität Göttingen, Faculty of Biology/JFB-Institute for Zoology and Anthropology, Department of Molecular Neurobiology of Behavior

\section{Prof. Dr. Andreas Stumpner}

Georg-August-Universität Göttingen, Faculty of Biology/JFB-Institute for Zoology and Anthropology, Department of Cellular Neurobiology

\section{Prof. Dr. Ernst A. Wimmer}

Georg-August-Universität Göttingen, Faculty of Biology/JFB-Institute for Zoology and Anthropology, Department of Developmental Biology 


\section{AFFIDAVIT}

I hereby declare that the dissertation entitled "Erythropoietin-mediated neuroprotection in insects" was written on my own and independently without any other aids and sources than indicated.

Göttingen, April 2016

Nataša Miljuš 

"Phantasie ist wichtiger als Wissen, denn Wissen ist Gegrenzt."

Albert Einstein 



\section{Table of content}

GENERAL INTRODUCTION AND AIMS........................................................ 1

I Signaling pathways involved in erythropoietin-mediated neuroprotection in Locusta migratoria in vitro .................................................... 5

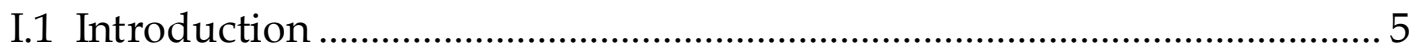

I.2 Material and Methods ....................................................................... 9

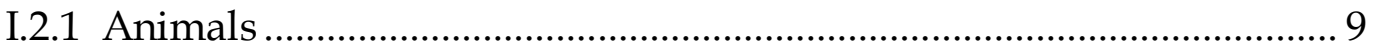

I.2.2 Preparation of primary cultures of locust brain cells ............................ 9

I.2.3 Induction of apoptosis ........................................................................... 11

I.2.4 Pharmacological treatment .................................................................... 11

I.2.5 Experimental design and timeline ..................................................... 12

I.2.6 Assessment of neuronal survival ......................................................... 13

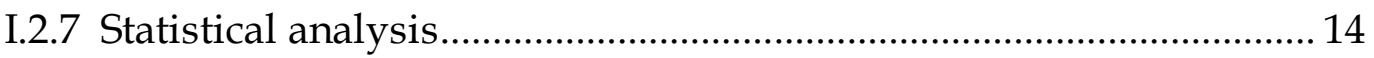

I.2.8 Anti-cleaved caspase 3 immunocy tochemistry.................................... 15

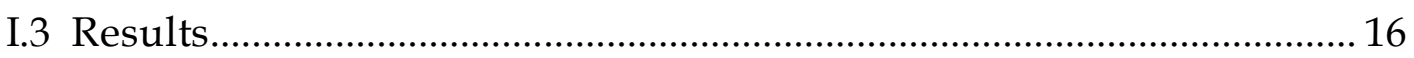

I.3.1 Characteristics of primary cultured locust brain cells in first five

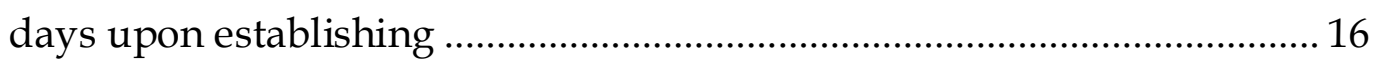

I.3.2 Epo prevents hypoxia- and H-7-induced cell death of locust brain

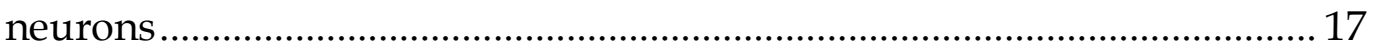

I.3.3 Epo-mediated neuroprotection depends on JAK/STAT activity ..... 19

I.3.4 Epo-mediated neuroprotection does not require PI3K and NF-kB

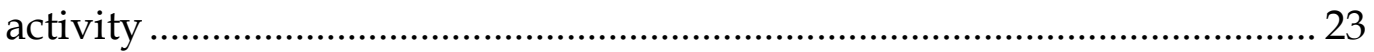

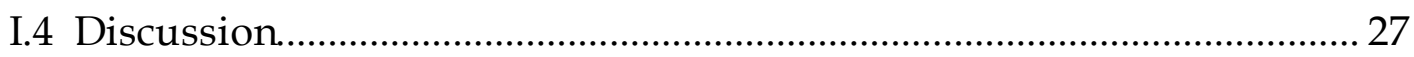

I.4.1 Epo-mediated protection of locust brain neurons from hypoxiaand H-7 induced apoptosis ....................................................................... 27

I.4.2 Requirement of JAK/STAT signaling for Epo-mediated protection of locust brain neurons................................................................................. 30

I.4.3 Contribution of PI3K and NF-kB pathways to Epo-mediated protection of locust brain neurons.

II Studies on the receptor that mediates neuroprotective properties of

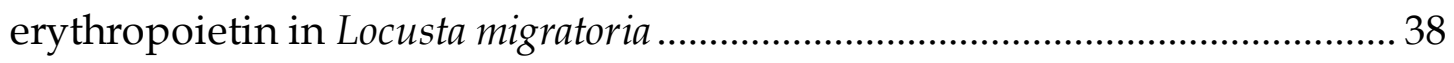

II.1 Introduction ............................................................................................. 38

II.2 Material and Methods ............................................................................ 42 
II.2.1 Examining the effect of the human Epo splice variant EV3 on neuronal survival during hypoxia-induced apoptosis.............................. 42

II.2.2 FM1-43 dye loading protocol and rhEpo-induced endocytosis...... 44

II.2.3 Analysis of endocytotic vesicles in primary cultured locust brain cells.

II.2.4 Anti-HRP immunocytochemistry and FM1-43 staining .................. 48

II.2.5 Labeling of rhEpo

II.2.6 Assessment of the labeling efficiency and purity of the rhEpofluorophore conjugate

II.2.7 Ligand-receptor binding and internalization assay in primary cultures of locust brain neurons using fluorescently labeled rhEpo 52

II.3 Results.

II.3.1 The non-erythropoietic Epo splice variant EV3 protects locust neurons from hypoxia-induced cell death.

II.3.2 Epo stimulates endocytosis in locust neurons 56

II.3.3 Determining the concentration of the rhEpo-fluorophore conjugate and the degree of labeling

II.3.4 Fluorescent scanning gel analysis and western blot analysis of the conjugate

II.3.5 Confocal microscopy analysis of primary cultured locust brain neurons exposed to labeled rhEpo.

II.4 Discussion

II.4.1 Endocytosis of rhEpo-stimulated receptor.

II.4.2 Chemical labeling of rhEpo to study its endocytosis after binding to its receptor

II.4.3 Protective effects of the non-erythropoietic Epo variant EV3 on the survival of primary cultured locust brain neurons challenged with hypoxia.

II.4.4 Pre-vertebrate evolution of a tissue-protective ligand-receptor system with structural and functional similarity to the mammalian Epo/Epo-receptor system.

III Potential effects of erythropoietin on insect neurogenesis

III.1 Introduction.

III.2 Material and Methods 86 
III.2.1 Manduca sexta cell line.....

III.2.1.1 Manduca sexta cell line maintenance. 86

III.2.1.2 Experimental protocol for studying the effect of Epo on proliferation and differentiation in the Manduca sexta cell line. 87

III.2.1.3 Detection of incorporated EdU via „click“ reaction . 87

III.2.1.4 Anti-HRP, anti-RePo, anti-synapsin and anti-fasciclin II immunochemistry. 88

III.2.1.5 Analysis of proliferation and differentiation 89

III.2.2 Primary cultures of locust optic lobe cells 90

III.2.2.1 Preparation of locust optic lobe primary cultures. 90

III.2.2.2 Experimental protocol for studying the effect of Epo on locust optic lobe cell proliferation and differentiation .......................... 91

III.2.2.3 EdU staining and anti-HRP immunocytochemistry ................ 91

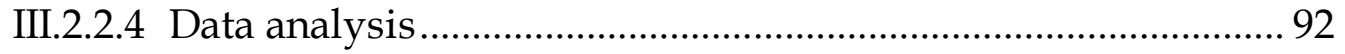

III.2.3 In vivo studies with Tribolium castaneum .......................................... 93

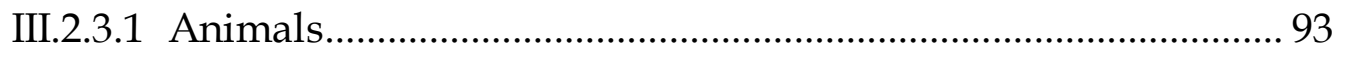

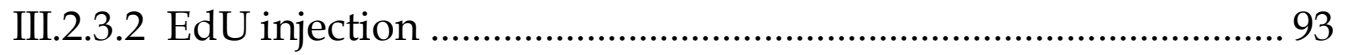

III.2.3.3 EdU- and immuno- staining of whole mount brains............... 94

III.2.3.4 Confocal microscopy and image processing............................ 95

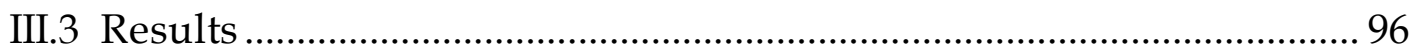

III.3.1 Characterization of Manduca sexta cell line ......................................96

III.3.2 Epo does not stimulate Manduca sexta progenitor cell proliferation and their differentiation into neurons expressing the neuronal marker

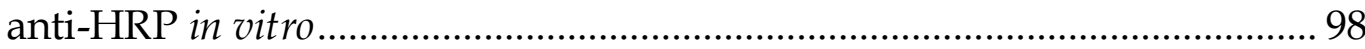

III.3.3 Epo has no effect on proliferation and differentiation of locust preadult optic lobe neuronal precursors in vitro ...................................... 101

III.3.4 In vivo studies on the proliferation of Tribolium castaneum

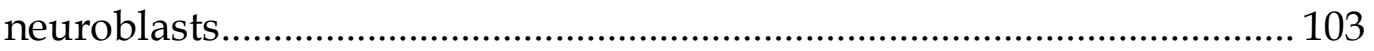

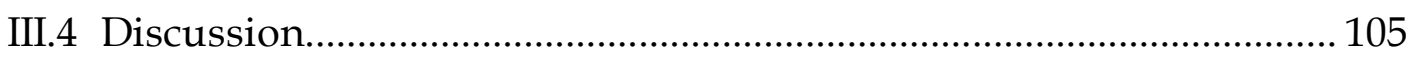

III.4.1 Epo-mediated regulation of neurogenesis ................................... 105

III.4.2 Epo does not induce neurogenesis in a neural cell line derived from embryonic tissue of Manduca sexta 106 
III.4.3 Epo does not support neurogenesis of optic lobe cells from nymphal Locusta migratoria brains in vitro

III.4.4 The potential involvement of Epo in adult neurogenesis of Tribolium castaneum could not be evaluated.

III.4.5 Injury-induced Epo-mediated neurogenesis ................................ 112

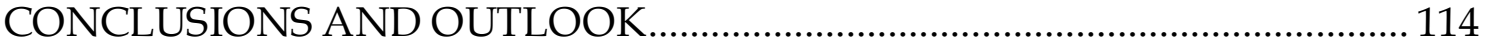

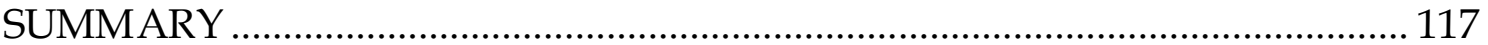

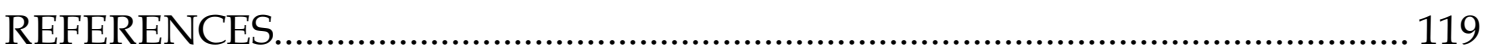

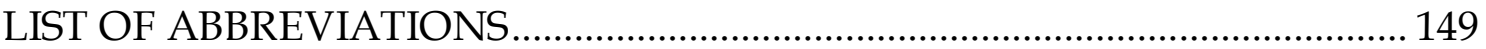

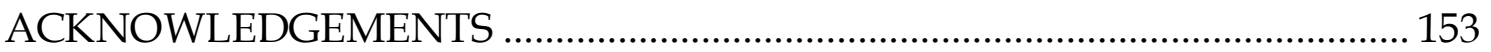

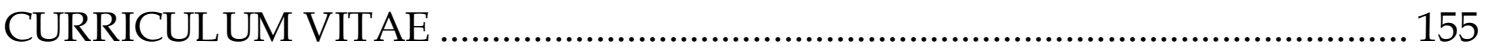

LIST OF PUBLICATIONS AND PRESENTATIONS ........................................... 157 


\section{List of Figures and Tables}

\section{Figures}

Figure 1. Establishment of primary cell cultures from locust brains

Figure 2. Schematic drawing of the experimental procedures to study the contribution of JAK, STAT, PI3K and NFKB to Epo-mediated protection of primary cultured locust brain neurons .....................13

Figure 3. Assessment of neuronal survival............................ 14

Figure 4. Primary cultured locust brain cells in first five days of

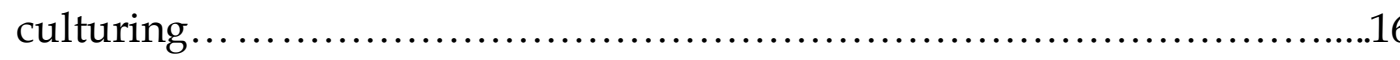

Figure 5. H-7 induces apoptosis in cultured locust brain neurons .........18

Figure 6. RhEpo (4 U/ml) protects primary cultured locust brain neurons from hypoxia- and H7- induced apoptotic cell death

Figure 7. DMSO has no effect on the survival of primary cultured locust brain neurons

Figure 8. RhEpo supports survival of locust neurons exposed to apoptosis-inducing stimuli via AG490-sensitive Janus kinase activity

Figure 9. Activation of sc-355979-sensitive STAT phosphorylation is required for the antiapoptotic effects of rhEpo in primary cultures of locust neurons...

Figure 10. RhEpo-mediated protection of primary cultured locust brain neurons is independent of LY294002-sensitive PI3K activity ...

Figure 11. Effects of different concentrations of LY294002 on the survival of primary cultured locust brain neurons....

Figure 12. RhEpo-mediated neuroprotection of locust neurons is independent of PDTC-sensitive IKB/NF-kB activity

Figure 13. Schematic drawing of the experimental procedure to evaluate a potential neuroprotective effect of EV3 on primary cultured Locusta migratoria brain neurons.

Figure 14. Structure of the FM1-43 dye molecule. .44 
Figure 15. Schematic drawing of the experimental procedure to study rhEpo-stimulated endocytosis in primary cultures of Locusta migratoria brain neurons

Figure 16. N-hydroxysuccinimde (NHS) ester-mediated chemical reaction between the amine-reactive group of the NHS ester-fluorophore complex and the primary amino group of a protein.... 49

Figure 17. Dose-dependent effect of the human Epo splice variant EV3 on the viability of primary cultured locust neurons exposed

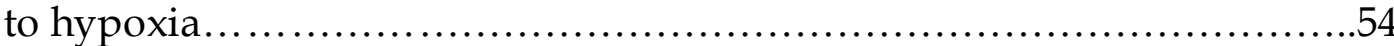

Figure 18. The human Epo variant EV3 protects primary cultured locust neurons from hypoxia-induced cell death

Figure 19. Physiologically intact locust brain neurons in vitro with endocytosed FM1-43 labeled vesicles, granular nucleic DAPI staining and anti-HRP positive "export" vesicles after 10 minutes exposure to $8 \mathrm{U} / \mathrm{ml}$ rhEpo

Figure 20: RhEpo stimulates endocytosis in cultured locust brain neurons .58

Figure 21: Automatic detection and quantification of FM1-43

labeled endocytotic vesicles....

Figure 22. Fluorescent scanning gel analysis and western blot analysis of the rhEpo-fluorophore conjugate.

Figure 23. Visualization of receptor-mediated endocytic uptake of fluorescently labeled rhEpo by primary cultured locust neurons using confocal microscopy...

Figure 24. Three individual optical sections from one stack of primary cultured locust brain neurons incubated with fluorescently labeled rhEpo and non-labeled rhEpo

Figure 25. Six individual optical sections from one stack of primary cultured locust brain neurons incubated with fluorescently labeled rhEpo................................................................

Figure 26. Anti-HRP immunostaining of the Manduca sexta cell line....... 96

Figure 27. Anti-synapsin and anti-fasciclin II immunostaining of the Manduca sexta cell line in conjunction with anti-HRP immunostaining......97 
Figure 28. Assessment of the effects of Epo on proliferation and differentiation in the Manduca sexta cell line.

Figure 29. Epo does not stimulate proliferation and differentiation of primary cultured locust optic lobe cells.............................102

Figure 30. In vivo EdU staining in the brain of Tribolium castaneum....... 104

\section{Tables}

Table 1. Comparison of characteristics of Epo-binding receptors in invertebrates and vertebrates... 



\section{GENERAL INTRODUCTION AND AIMS}

Long considered as a hormone with exclusive function in the regulation of vertebrate red blood cell production, erythropoietin (Epo) is today acknowledged as a pleiotropic cytokine that stimulates a wide spectrum of cellular responses in non-hematopoietic tissues providing protection against a variety of damaging stimuli. Beneficial functions of Epo outside of the hematopoietic system, especially in the nervous system, have been documented in many mammalian model systems (Sargin et al. 2010). Consequently, the neuroprotective potential of recombinant human Epo (rhEpo) has been probed in clinical pilot studies of human brain disorders, such as ischemic stroke (Ehrenreich et al. 2002; Ehrenreich et al. 2009) chronic schizophrenia (Ehrenreich et al. 2007; Wüstenberg et al. 2011) and chronic progressive multiple sclerosis (Ehrenreich et al. 2007), in which its beneficial effect was confirmed. However, chronical application of rhEpo was often accompanied with adverse side effects resulting from increased stimulation of erythropoiesis, such as thrombosis (Corwin et al. 2007). Over the last decade researchers invested enormous effort in understanding the cellular mechanisms that allow separation of tissue-protective from erythropoietic properties of Epo to enable the safe use of Epo as a therapeutic agent. The exact mechanism of the nonerythropoietic function of Epo however still remains to be clarified.

Orthologues of epo genes have been identified in vertebrates from fish to mammals (Chu et al. 2008) suggesting that Epo signaling was already established when vertebrates emerged approximately 420 million years ago. Hence, tissue protection against invading pathogens and other damaging stimuli may have been the original function of ancient Epo-like signaling (Brines \& Cerami 2005) common to vertebrates and probably some invertebrates. When adaptive immunity emerged in the vertebrate lineage, this system adapted an additional function in the regulation of erythropoiesis (Buchmann 2014). 
Studying invertebrate model systems in which erythropoiesis is absent might be advantageous in investigating the features of this evolutionary old tissueprotective Epo-like system. Insects typically use fewer different molecules for the regulation of their body functions, including adaptation of cellular processes to changing environments. Still, the involved molecules often have orthologues in vertebrates. Thus, with networks of signaling pathways that are usually less complex and less redundant compared to the pathways present in mammalian cells, insects might serve as a valuable model system to study intracellular mechanisms involved in Epo-mediated tissue-protection, independently of any erythropoiesis-mediated side effects. Studies on insects could provide important insights into the evolution of this tissue-protective system towards the one present in extant vertebrates. The understanding of differences between hematopoietic and tissue-protective properties of Epo is essential for the clinical translation of Epo-mediated beneficial treatments of central nervous damage and nervous system disorders.

Previous studies on acridid grasshoppers demonstrated neuroprotective and neuroregenerative effects of rhEpo. Namely, rhEpo increased the survival of primary cultured neurons from Locusta migratoria brains under normoxic and hypoxic conditions and accelerated neurite regeneration in vitro. In addition, rhEpo improved the reestablishment of sound localisation in the grasshopper Chorthippus biguttulus after transection of auditory nerve axons in vivo (Ostrowski et al. 2001).

The purpose of the present doctoral thesis was to study (in more detail) the cellular mechanisms of Epo-mediated protection of insect neurons.

The specific aim of the first part of the thesis was to characterize Epo-initiated transduction pathways involved in the protection of locust brain neurons exposed to apoptosis-inducing stimuli. In particular, I have studied whether these pathways are similar to those required for neuroprotection in mammals. 
The second aim of the thesis was to study functional characteristics of the Epobinding receptor on locust brain neurons. The main goal of this part was to examine whether activation of the receptor by rhEpo stimulates endocytosis in locust brain neurons. In addition, to obtain more information on the functional property of the Epo-binding receptor in locust neurons, I have studied whether the neuroprotective but non-hematopoietic human Epo splice variant EV3 can activate the receptor. The respective results are described in the second chapter of the thesis.

The third part of the project aimed to establish a suitable insect model system and protocols to study the potential involvement of Epo in insect neurogenesis. The objective was to analyse the ability of rhEpo to stimulate proliferation of stem-like progenitors and their differentiation into neurons. Three different insect preparations were analyzed in this respect. The results of these attempts are described in the third chapter of this thesis. 


\section{Signaling pathways involved in erythropoietin- mediated neuroprotection in Locusta migratoria in vitro}

\section{I.1 Introduction}

Erythropoietin (Epo) is the primary humoral mediator of hypoxic induction of vertebrate erythropoiesis (Bunn 2013). Apart from regulating erythropoiesis, evidence emerging over the last decade underlined the importance of Epo in mediating adaptive cellular responses triggered by diverse harmful stimuli in various non-hematopoietic mammalian tissues including the nervous system. Acting in a paracrine manner (Ruscher et al. 2002), Epo induces a wide range of cellular responses in the nervous system to protect and repair physiologically challenged or injured tissue. Beneficial functions include protection of neurons from apoptosis (Sirén et al. 2001a; Wen et al. 2002), glutamate excitotoxicity (Morishita et al. 1996) and oxidative damage (Chong et al. 2003; Kumral et al. 2005; Wu et al. 2007a), prevention of inflammatory responses (Agnello et al. 2002; Villa et al. 2003; Sättler et al. 2004; Chen et al. 2007a), promotion of angiogenesis (Wang et al. 2004) and neurogenesis (Shingo et al. 2001). Thus, by targeting not only injured mature neurons directly, but also neural progenitors, astrocytes, oligodendrocytes, microglia and endothelial cells (Byts \& Sirén 2009), Epo coordinates and orchestrates differential cell type-specific responses to promote healing and repair of injured nervous tissues.

As in the kidney, Epo production in the mammalian brain was observed to be hypoxia-inducible (Marti et al. 1996) and regulated via hypoxia-inducible factor (HIF) (Semenza \& Wang 1992). In addition to hypoxia other potentially harmful stimuli, such as hypoglycemia, insulin release and reactive oxygen species activate HIF and lead to increased expression of Epo (Masuda et al. 1997; Chandel et al. 1998). Epo exerts its physiological role by binding to a cell surface receptor. Epo receptor (EpoR) expressed on the surface of erythroid progenitor 
cells is a homodimeric cytokine class I receptor (Youssoufian et al. 1993). Signal transduction in erythropoiesis involves binding of one Epo molecule to an EpoR homodimer which leads to the activation of intracellular signaling cascades (Constantinescu et al. 1999). In contrast, tissue-protective functions of Epo are suggested to be mediated by binding to a heteromeric receptor, consisting of one hematopoietic EpoR monomer and another, probably cellspecific, receptor subunit. Potential heteromeric partners in non-hematopoietic tissues include one or more units of the beta common receptor chain (Brines et al. 2004) and the ephrine B4 receptor (Jackson et al. 2012; Debeljak et al. 2014).

The signaling pathways involved in neuroprotection overlap partially with those engaged in erythropoiesis. Both, neuroprotection and erythropoiesis are initiated by trans-phosphorylation and activation of receptor-associated tyrosine kinases of the Janus kinase 2 (JAK2) type as a result of conformational change in the receptor induced upon Epo binding (Witthuhn et al. 1993). Three principal downstream signaling pathways are subsequently activated. These include signal transducer and activator of transcription 5 (STAT5), phosphoinositol-3-kinase/protein kinase B (PI3K/Akt) and mitogen-activated protein kinase (MAPK) (Sirén et al. 2001a). STAT5 contributes to an universal antiapoptotic pathway activated in erythroid precursors (Silva et al. 1999; Socolovsky et al. 2001), neuronal cells (Sirén et al. 2001a) and other cell types. The PI3K/Akt pathway has been demonstrated to be involved in neural progenitor cell migration to the area of injury (Wang et al. 2006a) and regulation of endothelial responses (Chong et al. 2002). The MAPK pathway has been implicated in proliferation (Sui et al. 1998; Lawson et al. 2000) and differentiation of erythroid precursors (Klingmüller et al. 1997), as well as in reduction of inflammation (Brines 2014). All three pathways contribute to antiapoptotic effects of Epo through interference with apoptotic processes, accomplished either directly (e.g. via Akt that regulates the activity of caspases) (Digicaylioglu et al. 2004; Wu et al. 2007) or via activation of transcription factors that either suppress transcription of pro-apoptotic genes (e.g. bad) (Ruscher et al. 2002) or activate transcription of anti-apoptotic genes (e.g. $b c l-X_{L}$ ) 
(Wen et al. 2002). Epo-mediated neuroprotection in mammals additionally involves signaling pathways different from those involved in erythropoiesis, such as the NF-kB pathway (Digicaylioglu \& Lipton 2001; Liu et al. 2005).

The fact that Epo initiates similar transduction pathways to stimulate erythropoiesis and tissue protection suggests a common evolutionary origin of Epo signaling for both systems in vertebrates. Epo signaling seems to be common to all vertebrates since homologues of the human epo gene have been identified in various mammalian (Wen et al., 1993), amphibian (NogawaKosaka et al. 2010) and fish species (Chou et al. 2004; Chu et al. 2007; Chu et al. 2008; Ostrowski et al. 2011). In contrast, orthologues of epo and epor genes could so far not be identified in any invertebrate species, while downstream components of mammalian Epo signaling pathways, namely JAK, STAT, PI3K, Akt, NF-kB are present in invertebrates, including various insect species (Ghosh et al. 1998; Scanga et al. 2000; Arbouzova \& Zeidler 2006). The significance of these signaling networks in insect cells is reflected by their requirement for normal developmental processes (Bina \& Zeidler 2009) and for innate immune responses against invading pathogens (Agaisse et al. 2003). Despite the absence of Epo and EpoR orthologues in insects, a previous study on grasshoppers found neuroprotective and neuroregenerative effects of rhEpo in vitro and in vivo (Ostrowski et al. 2011). Hence, neuroprotection or even general tissue protection might have been the original function of an ancient Epo/EpoR-like signaling system that evolved in a common ancestor of vertebrates and insects as a part of innate immunity. With the evolution of specialized oxygen carrying erythrocytes at the basis of the vertebrate lineage the Epo/EpoR system was subsequently adapted for its role in vertebrate erythropoiesis (Svoboda \& Bartunek 2015).

Although orthologues of mammalian components of Epo-stimulated intracellular transduction pathways have been described in insects (most completely in Drosophila melanogaster), their requirement for Epo-mediated protection of insect neurons have not been studied so far. Therefore, the aim of 
the first part of this thesis was to examine whether signaling pathways involved in Epo-mediated protection of insect (locust) neurons are similar to those required for neuroprotection in mammals. Similarities in signaling cascades leading to Epo-mediated neuroprotection between mammals and insects would thus provide further support for the hypothesis of a pre-vertebrate evolution of Epo/EpoR-like signaling with an original function in cell and tissue protection. 


\section{I.2 Material and Methods}

\section{I.2.1 Animals}

Experiments were carried out with fourth-instar nymphs of Locusta migratoria purchased from a commercial breeder (Hintze, Berlin, Germany). Until usage, animals were maintained in colonies at $22-25^{\circ} \mathrm{C}$ on a $12 / 12$-hours day/night cycle.

\section{I.2.2 Preparation of primary cultures of locust brain cells}

Before experimental preparation of locust brains, round coverslips $(\varnothing 11 \mathrm{~mm}, \mathrm{~A}$. Hartenstein, Würzburg) were placed carefully with forceps into sterile plastic culture dishes ( $\varnothing 35 \mathrm{~mm}$, Corning, NY, USA) and coated with a solution of Concanavalin A (ConA, Sigma-Aldrich Chemie, Schnelldorf, Germany; 100 $\mu \mathrm{l}$ /coverslip). ConA is a lectin that binds specifically to carbohydrate fractions of cell membrane glycoproteins and glycolipids facilitating the adhesion of the cells to the substrate (Hardman \& Ainsworth 1972). After approximately $2 \mathrm{~h}$ and shortly before the plating of the cell suspension, ConA was removed and coverslips were carefully washed 3 times with autoclaved phosphate-buffered saline (PBS, pH 6.9).

Brain dissection was carried out under a stereo microscope (Olympus, Mod. SZ61, Tokyo, Japan). Locust heads were separated from their bodies and pinned to the surface of a sylgard-coated plate. The frontal head cuticle was cut in three directions using fine scissors (from mouth region towards the eyes in dorsal direction, followed by two diagonal cuts towards the eyes in lateral direction, Figure 1). The head capsule was opened using fine forceps to carefully separate connective tissue lying just beneath the cuticle. Locust brains without optic lobes were dissected out from head capsules, collected in sterile culture dishes filled with Leibovitz`s L-15 Medium (L15, Gibco, Life Technologies, Thermo Fisher Scientific, Braunschweig, Germany) supplemented with 0.5\% gentamicin (GM, BioReagent, Sigma-Aldrich) and cleared from remaining trachea under sterile conditions. All following steps were carried out in a clean bench (Thermo 
Fisher Scientific, Mod. Safe 2020). Cleaned brains were pooled into a single culture dish and rinsed three times with L15/0.5\% GM. For enzymatic digestion of extracellular matrix proteins, brains were incubated in L15/0.5\% GM containing a $1 \mathrm{mg} / \mathrm{ml}$ mixture of collagenase/dispase (Sigma-Aldrich) for 15 minutes (min) in an incubator (model B6120, Heraeus Instruments, Hanau, Germany) at $27^{\circ} \mathrm{C}$. After three short washings with Hanks' Balanced Salt Solution (HBSS, Gibco, Thermo Fisher Scientific), cells were mechanically dissociated by gentle trituration of the brain tissue via repeated passages through the tip of a $1 \mathrm{ml}$ Eppendorf pipette (20 times), followed by further repeated passages through the tip of a $100 \mu$ Eppendorf pipette (again for 20 times). After $1 \mathrm{~min}$ spinning of triturated tissue at $3000 \mathrm{x} \mathrm{g}$ with a bench rotor (Quick Spin QS7000, Süd-Laborbedarf, Gauting, Germany), the supernatant was discarded and the pellet of dispersed brain cells resuspended in L15/0.5\% GM (50 $\mu 1$ per brain). The cell suspension (consisting of the pooled cells from a larger number of locust brains) was equally distributed onto previously prepared ConA-coated coverslips (100 $\mu \mathrm{l} /$ coverslip). This procedure assured that each of the cultures used in the same experiment contained an equal density of cells (around 2 brains/coverslip) that originated from the same pool of dissociated brains. Cells were allowed to adhere to the coverslips for $2 \mathrm{~h}$ at room temperature (RT), (under the clean bench), before L15/0.5\% GM medium supplemented with fetal bovine serum gold (FBSG, PAA laboratories, GE Healthcare Life Sciences, Freiburg, Germany) at a final concentration of 5\% (v/v) (L15/0.5\%GM/5\%FBSG) was added to the culture dishes (2 ml/culture dish). The primary cultures of locust brain cells were placed in an incubator at $27^{\circ} \mathrm{C}$ in a humidified atmosphere and maintained (usually for 5 days) until experiments were performed. The medium was replaced with fresh L15/0.5\%GM/5\%FBSG every 2 days and cultures were inspected for their growth, viability and possible contamination every day with an inverted light microscope with phase-contrast (CKX41, Olympus). 


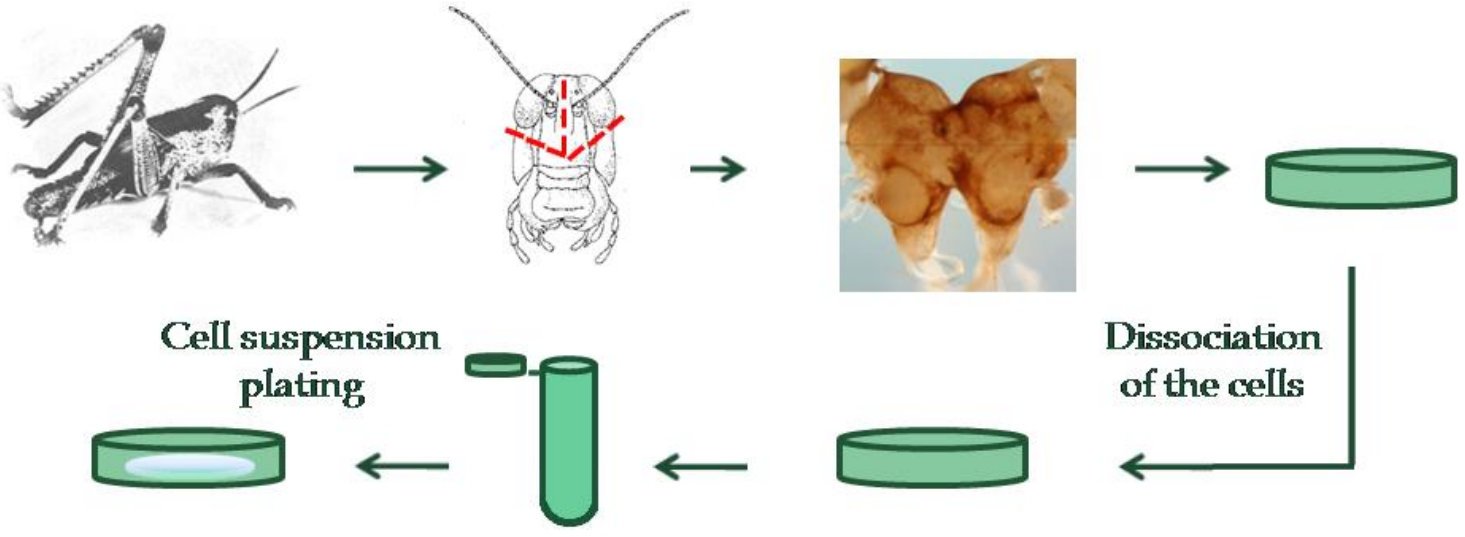

Figure 1. Establishment of primary cell cultures from locust brains. Locust brains were dissected, pooled and their dissociated cells plated onto coverslips and cultured in dishes with medium.

\section{I.2.3 Induction of apoptosis}

Apoptosis of primary cultured brain cells was induced by hypoxia or H-7 treatment. For hypoxia experiments cultures were placed in airproof chambers (22.5 $\times 4.5 \times 4 \mathrm{~cm}$, workshop of our institute), equipped with an oxygen analyzer (Greisinger GOX 100, Conrad Electronics, Hirschau, Germany). Oxygen $\left(\mathrm{O}_{2}\right)$ was removed by floating the chamber with nitrogen $\left(\mathrm{N}_{2}\right)$. $\mathrm{O}_{2}$ level was maintained at $\leq 2 \%$. Hypoxia was applied to the cell cultures for $36 \mathrm{~h}$ at RT. Control cultures were kept under normoxic conditions for the same period. To chemically induce apoptosis, cells were incubated with 1-(5 isoquinolinesulfonyl)-2-methylpiperazine (H-7, Sigma-Aldrich) at a final concentration of $50 \mu \mathrm{M}$ for $18 \mathrm{~h}$ at $27^{\circ} \mathrm{C}$. Untreated control cultures were maintained at the same temperature for the same period.

\section{I.2.4 Pharmacological treatment}

To examine Epo-initiated intracellular signaling pathways that mediate protection from experimentally induced apoptosis, cell cultures were treated with combinations of $4 \mathrm{U} / \mathrm{ml}$ rhEpo (NeoRecormon, Roche, Welwyn Garden City, UK) and membrane permeable inhibitors of particular transduction 
mechanisms diluted in serum-free medium. $4 \mathrm{U} / \mathrm{ml}$ rhEpo has previously been determined as being most effective to support the survival of locust brain neurons in vitro (Ostrowski et al. 2011), with both higher and lower concentrations being less beneficial. Stock solutions of the JAK2 inhibitor AG490 (Cayman Chemicals, MI, USA), the STAT5 inhibitor sc-355979 (New England BioLabs, Frankfurt am Main, Germany) and the PI3K inhibitor LY294002 (New England BioLabs) were first dissolved in dimethyl sulfoxide (DMSO, Sigma-Aldrich) (final concentration of DMSO in cultures was $<1 \% ; 1 \%$ DMSO does not affect the viability of the primary cultured brain cells; see Fig. 7) and then diluted with culture media to give final experimental concentrations of $5 \mu \mathrm{M}$. The NFKB/IкB inhibitor, pyrrolidine dithiocarbamate (PDTC, Sigma-Aldrich), was directly dissolved in culture media to a final concentration of $100 \mu \mathrm{M}$. All inhibitors (except PDTC) were applied $1 \mathrm{~h}$ before (hypoxia experiments) or together with ( $\mathrm{H}-7$ experiments) the addition of rhEpo. To secure efficient inhibition of NFKB/ ІкB activation PDTC was applied at least 1,5 h before addition of rhEpo (Schreck et al. 1992). Additional control experiments were performed with the rhEpo solvent ( $3 \mathrm{mg}$ polysorbate 80,50 mg glycine, $43.8 \mathrm{mg} \mathrm{NaCl}, 5.13 \mathrm{mg} \mathrm{NaHPO} \times 2 \mathrm{H}_{2} \mathrm{O}, 22.3 \mathrm{mg} \mathrm{Na} 2 \mathrm{HPO}_{4} \times 2 \mathrm{H}_{2} \mathrm{O}$ in $10 \mathrm{ml} \mathrm{H}_{2} \mathrm{O}$ ) added in the same volume as rhEpo in experimental treatment groups.

\section{I.2.5 Experimental design and timeline}

Two approaches were used to study intracellular signaling pathways involved in Epo-mediated protection from experimentally induced apoptosis (Fig. 2). Five days after establishment of primary neuronal cultures full medium was replaced with serum-free medium supplemented with or without rhEpo and with or without an inhibitor of a particular signaling molecule (see I.2.4). In the first approach, cultures were maintained for $12 \mathrm{~h}$ in normoxia and thereafter exposed to hypoxic atmosphere for $36 \mathrm{~h}$. After reoxygenation, cell cultures were maintained for $12 \mathrm{~h}$ in normal atmosphere before fixation and subsequent analysis of cell survival. In the second approach, after application of rhEpo 
and/or a specific inhibitor or serum-free medium only (control) (see I.2.4) cells were maintained for $10 \mathrm{~h}$ at $27^{\circ} \mathrm{C}$. Subsequently they were exposed to $\mathrm{H}-7$ treatment for $18 \mathrm{~h}$ at $27^{\circ} \mathrm{C}$. Afterwards cells were fixed and labeled for analysis.
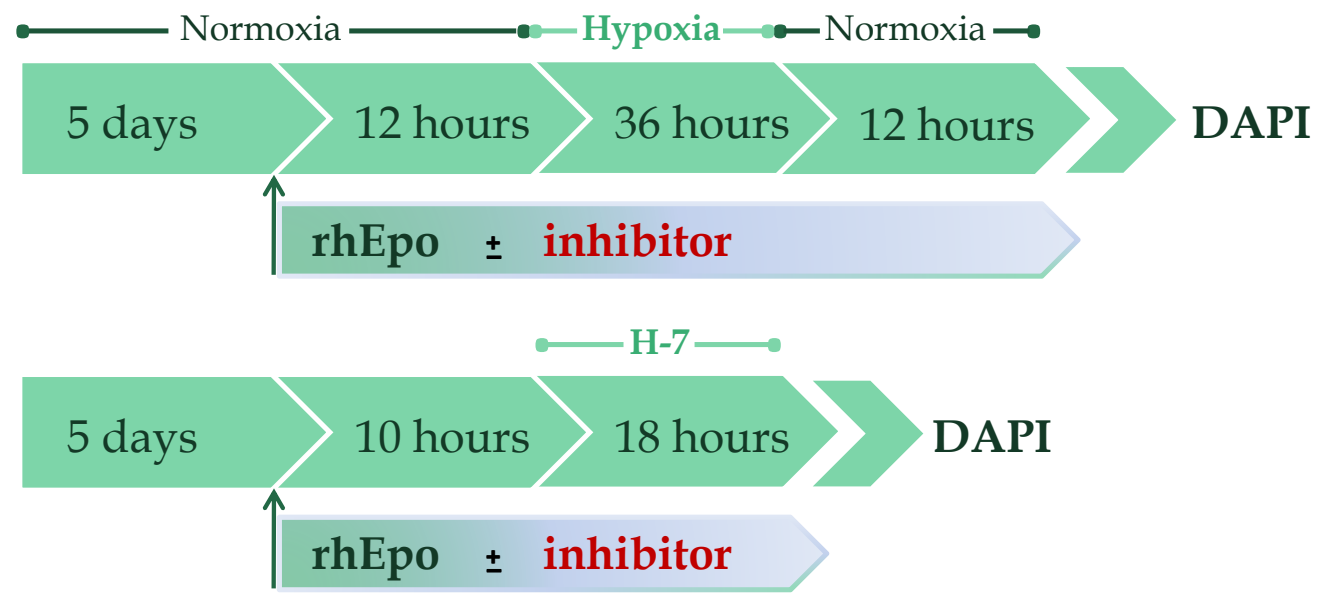

Figure 2. Schematic drawing of the experimental procedures to study the contribution of JAK, STAT, PI3K and NFKB to Epo-mediated protection of primary cultured locust brain neurons. Inhibitors are: AG490, sc355979, LY294002 and PDTC.

\section{I.2.6 Assessment of neuronal survival}

To analyze the survival of primary cultured neurons the cultures were fixed with $4 \%$ paraformaldehyde in $0.1 \mathrm{M}$ phosphate buffer for $30 \mathrm{~min}$ at RT. After three 5-min washes with PBS ( $\mathrm{pH}$ 6.9) and two 5-min washes with PBS containing $0.1 \%$ Triton-X-100, the cells were incubated with 4,6-diamidino-2phenylindole dihydrochloride (DAPI, Sigma-Aldrich) dissolved in PBS at a final concentration of $100 \mu \mathrm{g} / \mathrm{ml}$ for $30 \mathrm{~min}$ at RT in the dark. Excess dye was removed by several washes with PBS and the cell-containing coverslips were mounted on microscopic slides in 1,4-diazobicyclo [2.2.2] octane (DABCO, Carl Roth, Karlsruhe, Germany). Analysis of DAPI fluorescence was performed using an epifluorescence microscope (Zeiss Axioskop; 40x objective) equipped with a Spot CCD camera (Invisitron, Puchheim, Germany). From each culture, two continuous rows of non-overlapping photographs (usually 50-70 per 
culture) to the right and the left of the center along the entire coverslip were taken. Post-treatment survival of the cells was assessed based on the DAPI staining pattern (Fig. 3), from which the proportion of living cells relative to the total cell number was determined. DAPI is a fluorescent stain that preferentially attaches to $\mathrm{A}-\mathrm{T}$ rich sequences in the minor grove of double-stranded DNA (Kapuściński \& Szer 1979). One molecule of dye usually extends along three base-pairs, resulting in characteristic discontinuous staining pattern of chromatin in viable cell nuclei. Unlike viable cells, dying cells are characterized by bright, compact DAPI staining, reflecting condensed chromatin structure (Gocht et al. 2009).

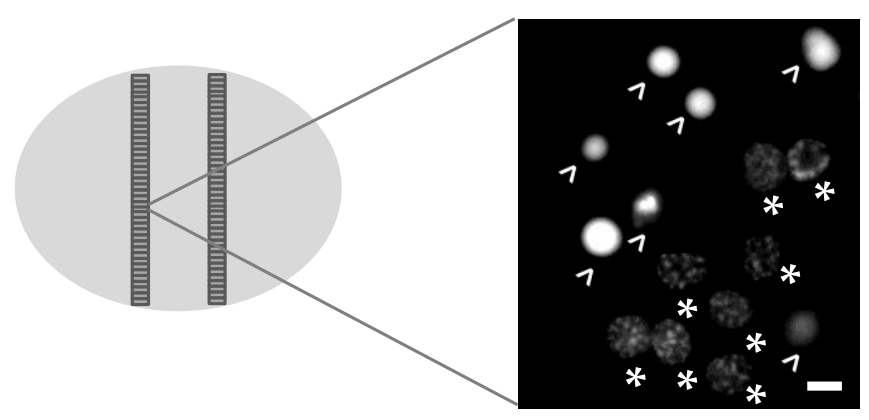

Figure 3. Assessment of neuronal survival. Left: after experimental treatment and nuclear labeling with DAPI, two continuous rows of non-overlapping photographs (usually 60 per culture) extending over the entire coverslip were recorded. Right: nuclei of living cells are characterized by a discontinuous patchy pattern of DAPI staining $\left(^{*}\right)$, whereas nuclei of late apoptotic or dead cells are condensed and uniformly labeled with DAPI (>). Scale bar: $10 \mu \mathrm{m}$.

\section{I.2.7 Statistical analysis}

In order to compare different experiments of the same type, the portion of living cells in different treatment groups of one experiment was normalized to the portion of living cells in the untreated control culture (set as 100\%). The relative portion of living cells in [\%] is displayed in typical box plot graphs. Boxes contained $50 \%$ of values around the median. Spread of upper and lower $25 \%$ of values is displayed in the whiskers. The software package OriginPro (version 8.5, Origin Lab Corporation) was used to generate initial diagrams and to perform statistical analyses. Data sets of most experiments were not normally 
distributed (Shapiro-Wilk-Test). Therefore, all experiments were evaluated with (more conservative) non-parametrical tests. The Kruskal-Wallis test was used to identify differences in data population distributions and the MannWhitney $U$ test for unmatched samples was used to evaluate the differences between two selected groups. $P$ values were corrected with the BenjaminiHochberg procedure (Benjamini \& Hochberg, 1995; Groppe et al., 2011). For calculations we used the Matlab implementation of Benjamini and Hochberg's procedure by David M. Groppe, Dept. of Cognitive Science, University of California, San Diego (http://www.mathworks.com/matlabcentral/ fileexchange/29274-mass univariate-erp-toolbox/content/fdr_bh.m). $P$ values smaller than 0.05 were considered as significantly different.

\section{I.2.8 Anti-cleaved caspase 3 immunocytochemistry}

Primary cell cultures from locust brains were fixed with $4 \%$ paraformaldehyde dissolved in $0.1 \mathrm{M}$ phosphate buffer for $15 \mathrm{~min}$ at RT. After three washes in PBS and three washes in PBS with 1\% Triton-X-100 (each for $5 \mathrm{~min}$ ), cells were incubated for $1 \mathrm{~h}$ with blocking solution containing 5\% normal goat serum (GE Healthcare Life Sciences) and $0.25 \%$ bovine serum albumin (MP Biomedicals, Heidelberg, Germany) dissolved in PBS with 0.1\% Triton. Primary antibody anti-cleaved caspase 3 from rabbit (Calbiochem Merck, UK, dilution 1:300, v/v, in blocking buffer) was incubated over night at $4^{\circ} \mathrm{C}$. After several washes in PBS, cells were treated with RNase (Sigma-Aldrich, $100 \mu \mathrm{l} / \mathrm{ml}$ PBS for $10 \mathrm{~min}$ at $37^{\circ} \mathrm{C}$ ) to degrade cytosolic RNA. After several washes with PBS, secondary antibody Cy2-coupled goat anti rabbit (Dianova, Hamburg, Germany, dilution 1:200 in blocking buffer) together with propidium iodide (Sigma-Aldrich, 1:1000) was applied for $2 \mathrm{~h}$ at RT. After washing away excess antibody and propidium iodide, coverslips with labeled cells were treated with 1:1 PBS/glycerol and mounted on microscopic slides. Stainings were analyzed by confocal fluorescence microscopy (Leica TCS SP2, Leica Microsystems, Wetzlar, Germany). 


\section{I.3 Results}

\section{I.3.1 Characteristics of primary cultured locust brain cells in first five days upon establishing}

As previously demonstrated (Gocht et al. 2009), chemical and mechanical dissociation of locust brain tissue during the preparation of locust brain cell cultures causes disruption of cellular processes and extensive cell loss during the first days of culturing. The remnants of irreversibly damaged cells disintegrate, detach from the substrate and are removed from the cultures during the procedure of medium exchange. Neurons which were not irreversibly damaged regenerate their neurites in vitro, a process that is supported by trophic factors contained in the serum. In contrast, survival of glia is not supported by the type of serum used in our studies and the number of glial cells rapidly decreases with culturing time. Consequently, after 5 days in vitro primary cell cultures from locust brains contain almost exclusively neurons (Fig. 4).
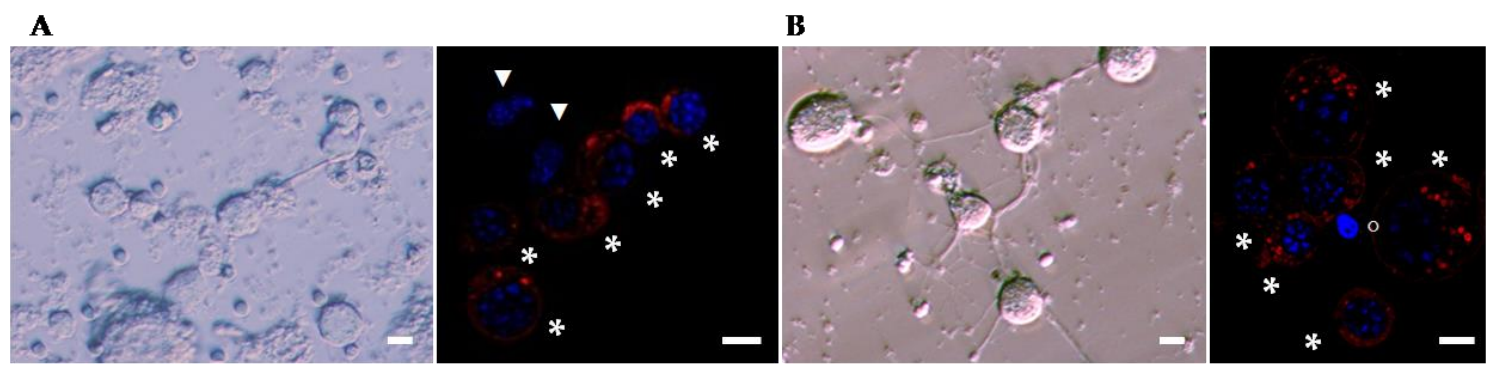

Figure 4. Primary cultured locust brain cells in first five days of culturing. (A) Left: phasecontrast image of a culture 24 hours after establishment. Viable cells have adhered to the substrate, while severely damaged cells have shrunk and reduced their size. Remnants of dead cells are present throughout the culture. Right: anti-HRP immunostaining (red) 24 hours after establishment of primary locust brain cells culture. Six viable cells are positive for the panneuronal marker HRP $\left({ }^{*}\right)$, and two viable cells are anti HRP-negative $(\boldsymbol{\nabla})$. Blue: DAPI staining of nuclei. (B) Phase-con trast image (left) and fluorescent image (right) of primary cultured locust brain cells five days after establishment. Left: viable cells have extended their neurites and established anatomical contacts. Right: six viable HRP-positive cells $\left(^{*}\right)$ surrounding one dead cell $\left(^{\circ}\right)$. Scale bars: $10 \mu \mathrm{m}$. 
As previously shown, after 4 days in vitro less than $5 \%$ of surviving cells are negative to anti-HRP immunolabeling indicating their non-neuronal type (Gocht et al. 2009). To exclude potential interference of glia-derived factors on neuronal survival and to allow comparison between independent experiments, all experiments were conducted 5 days after establishment of primary locust brain cell cultures.

\section{I.3.2 Epo prevents hypoxia- and H-7-induced cell death of locust brain neurons}

After 5 days in vitro, full medium was replaced with serum-free medium to eliminate effects mediated by factors from the serum. Cell cultures were subjected to the experimental treatments described by schematic drawings in the upper parts of the respective figures. To initiate apoptotic cell death primary cultured locust brain cells were either exposed to hypoxic atmosphere $\left(\mathrm{O}_{2}\right.$ level $\left.<2 \%, 36 \mathrm{~h}\right)$ or chemically treated with $\mathrm{H}-7(50 \mu \mathrm{M}, 18 \mathrm{~h})$. Both stimuli have previously been demonstrated to induce apoptosis in vertebrate and invertebrate cells. The process of programmed cell death, apoptosis, is characterized by activation of caspases and distinct morphological features that include DNA fragmentation accompanied with chromatin condensation and ultimately loss of the entire cytosolic compartments (Elmore 2007). The most prominent feature of apoptosis, chromatin condensation, was detected in primary cultures of locust brain cells using DAPI staining (Figs. 3, 4). This detection strongly agreed with the results of analysis of other signs of apoptosis obtained upon propidium iodide staining and anti-cleaved caspase 3 immunostaining (Fig. 5). Activated caspase 3 was only detected in apoptotic cells that still contained intact cytomembranes to enclose the cytoplasmic compartment that contained the caspase. 

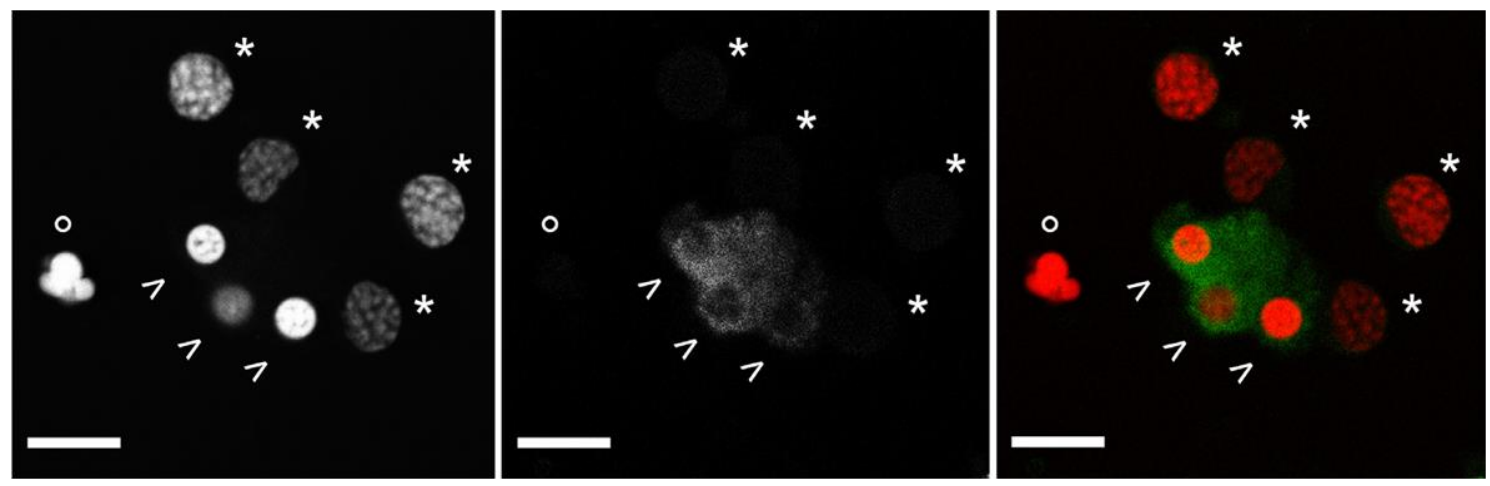

Figure 5. H-7 induces apoptosis in cultured locust brain neurons. Left: propidium iodide labels chromatin DNA structures in large nuclei of intact cells $(*)$, condensed nuclei with fragmented DNA in apoptotic cells $(>)$ and nuclear remnants with completely fragmented DNA $\left(^{\circ}\right)$. Middle: Anti-cleaved caspase 3 immunoreactivity in the cytoplasm of 3 apoptotic cells (>). Right: overlay of propidium iodide labeling (red) and anti-cleaved caspase 3 immunofluorescence (green). Apoptotic cells (>) contain condensed nuclei with largely fragmented DNA along with the presence of activated caspase 3 in their cytosol. In contrast, cytoplasm of intact cells $\left(^{*}\right)$ with discontinuous propidium iodide labeling is free of activated caspase 3 . Nuclear remnants of late apoptotic cells with completely fragmented DNA $\left(^{\circ}\right)$ have lost their cytosolic compartments. Scale bar: $15 \mu \mathrm{m}$.

As shown in Figure 6A, compared to control cultures that were kept under normoxic conditions (neuronal survival normalized to $100 \%$ ), hypoxic treatment reduced the portion of surviving neurons (median of surviving cells: 61.34\%; difference to control $\left.P=2^{*} 10^{-6}\right)$. Similarly, compared to untreated control cultures, treatment with H-7 reduced neuronal survival (median of surviving cells: $77.93 \%$; difference to control $P=0.0029$ ) (Fig. 6B). Pre-treatment with rhEpo starting before induction of apoptosis by hypoxia or H-7 completely prevented these deleterious effects. RhEpo increased neuronal survival in both, hypoxiaexposed (median: 111.88\%, $P=2^{*} 10^{-6}$ ) and H-7-exposed (median: 108.19\%, $P=0.0029)$ locust cultures to levels that were similar to those detected in untreated control cultures. In contrast, solvent of rhEpo exerted no beneficial effect on cell survival during H-7 treatment (median: 76.5\% ) (Fig. 6B), indicating that the neuroprotection was exclusively achieved through rhEpo and not by ingredients of its solvent. Moreover, rhEpo did not significantly affect cell survival in cultures that were not challenged by apoptosis-inducing stimuli (median: 96.71\%) (Fig. 6B). 
A
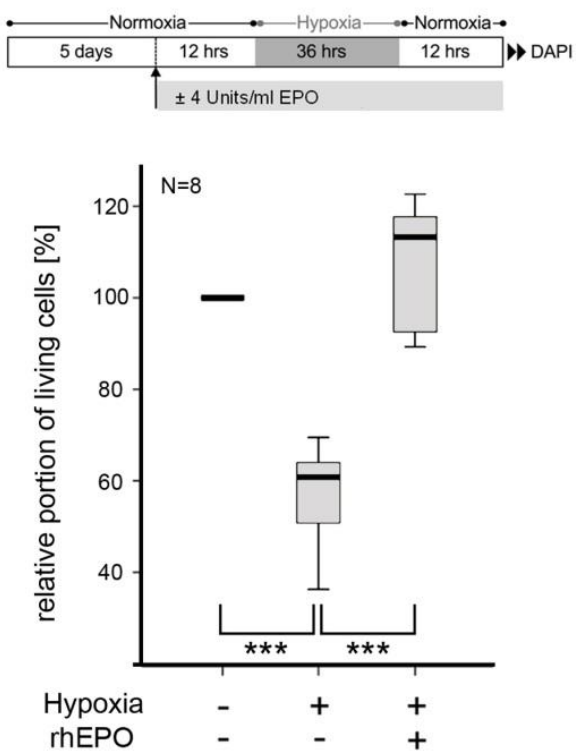

B
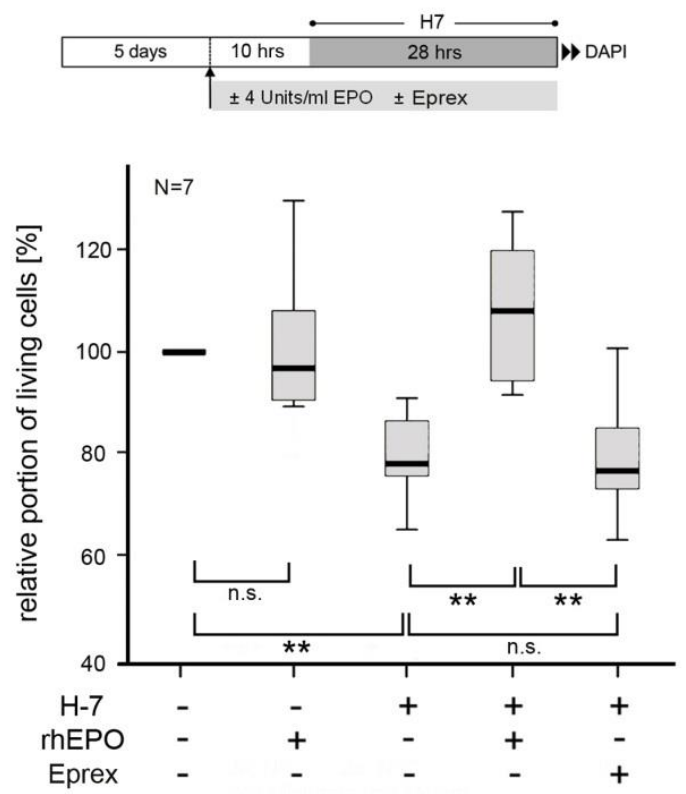

Figure 6. RhEpo $(4 \mathrm{U} / \mathrm{ml})$ protects primary cultured locust brain neurons from hypoxia - (A) and H7- (B) induced apoptotic cell death. Cell viability was normalized to untreated conditions $(=100 \%)$. While neuronal survival decreased upon exposure to hypoxia $\left({ }^{* * *} P<0.001\right)$ and $\mathrm{H}-7$ $\left({ }^{* *} P<0.01\right)$, application of rhEpo suppressed both hypoxia- $\left.{ }^{* * *} P<0.001, N=8\right)$ and H-7- $\left({ }^{* *} P<0.0\right.$, $\mathrm{N}=7$ ) -induced apoptosis. RhEpo has no beneficial effect on cultures not exposed to apoptotic stimuli. RhEpo's solvent (Eprex) has no neuroprotective effect. Statistics: Kruskal-Wallis ANOVA with Mann-Whitney $U$-test and Benjamini-Hochberg-correction. ${ }^{* *} P<0.01,{ }^{* * *} P<0.001$. Schematics of the experimental procedures are illustrated in the upper parts of the figure.

\section{I.3.3 Epo-mediated neuroprotection depends on JAK/STAT activity}

To explore the contribution of different signaling pathways to Epo-mediated antiapoptotic effects in locust neurons, we used a pharmacological approach in which a component of a particular signaling pathway was selectively inhibited. Membrane permeable inhibitors of particular signaling pathways were dissolved in DMSO and thus it was necessary to test first whether DMSO applied in the amount corresponding to the amount of soluble inhibitor (less than $1 \%$ ) affects cellular survival. As it has been determined that 1\% DMSO does not affect viability of primary cultured locust brain cells (Fig. 7), we proceeded with pharmacological treatments. 


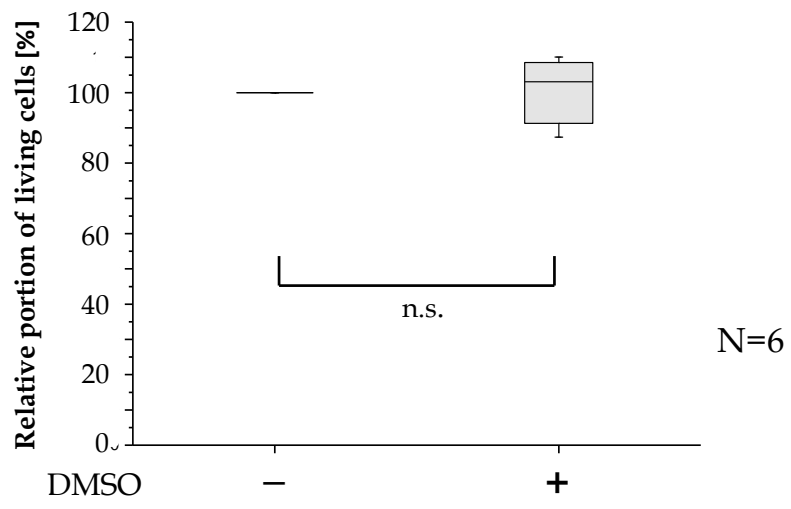

Figure 7. DMSO has no effect on the survival of primary cultured locust brain neurons. Cultures were exposed to DMSO for 60 hours. Concentration of DMSO in culture media: $1 \%$. ( $P=0.36$, Mann-Whitney $U$ test. N.s. not significant.)

It has been shown that binding of Epo to its preformed dimeric receptor on the cell surface of both erythropoietic and non-hematopoietic mammalian cells leads to a conformational change that causes activation of receptor-associated tyrosine kinase JAK2 (Digicaylioglu \& Lipton 2001; Remy 1999; Witthuhn et al. 1993) by transphosphorylation. To examine whether rhEpo-mediated protection of locust neurons requires JAK, the membrane permeable JAK inhibitor AG-490 was used. As described above, hypoxia reduced the survival of cultured neurons and hypoxia-induced cell death was prevented in the presence of Epo (Fig. 8A). Application of $5 \mu \mathrm{M}$ AG-490 did not further reduce the number of surviving neurons under hypoxic conditions (median: $49.54 \%$ ) compared to the survival of neurons exposed to hypoxia only (median: $60.16 \%, P=0.39$, n.s.), but completely abolished antiapoptotic effects of rhEpo on hypoxia-exposed cell cultures. Neuronal survival in cultures that were co-incubated with AG-490 and rhEpo and subjected to hypoxia was significantly reduced (median: 55.84\%) compared to the survival of hypoxia-treated neuronal cultures rescued with rhEpo (median: $110.55 \%, P=8.65^{*} 10^{-6}$ ) and to neuronal survival under normoxia $\left(P=1.92^{*} 10^{-7}\right)$, but did not differ from viability of neurons exposed to hypoxia only (median: 60.16\%, $P=0.69$ ). A similar effect of AG-490 was obtained when apoptosis was induced by H-7. As shown in Figure 8B, upon induction of 
apoptosis by H-7 (median: 68.89\%), rhEpo supported the survival of primary cultured locust neurons (median: 107.13\%, $P=0.046$ ). The neuroprotective effect of rhEpo was blocked by the presence of AG-490, as neuronal survival significantly decreased in cultures treated with the combination of H-7, AG-490 and rhEpo (median: 25.03\%), in comparison to cultures treated with $\mathrm{H}-7$ and rhEpo $(P=0.0089)$, but was not significantly different from the survival of neurons in cultures treated with $\mathrm{H}-7$ only $(P=0.058)$. In summary, these results indicate that the antiapoptotic effects of rhEpo on cultured locust brain neurons are mediated by AG-490-sensitive JAK activation.

A
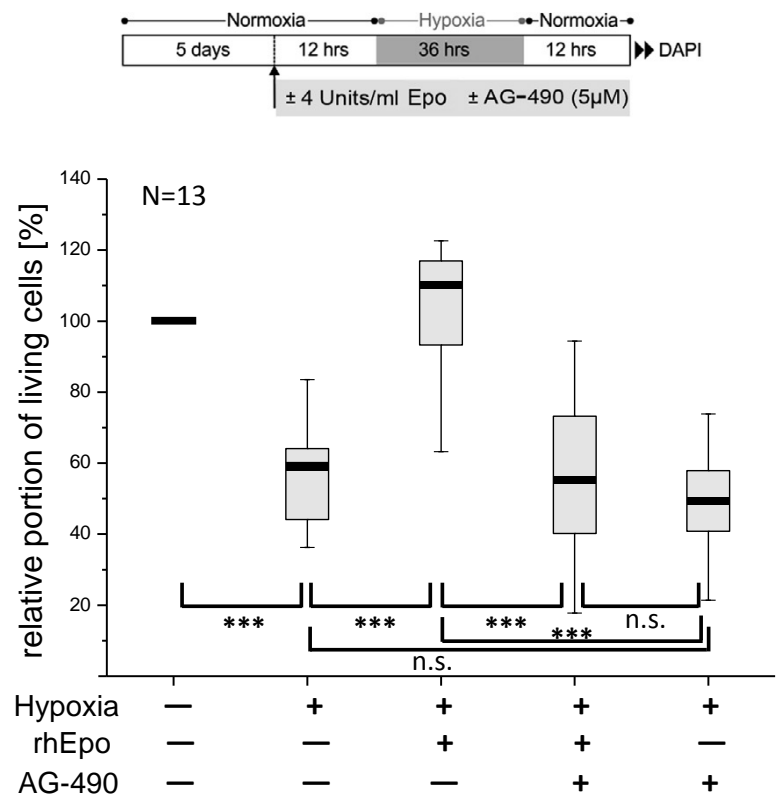

B
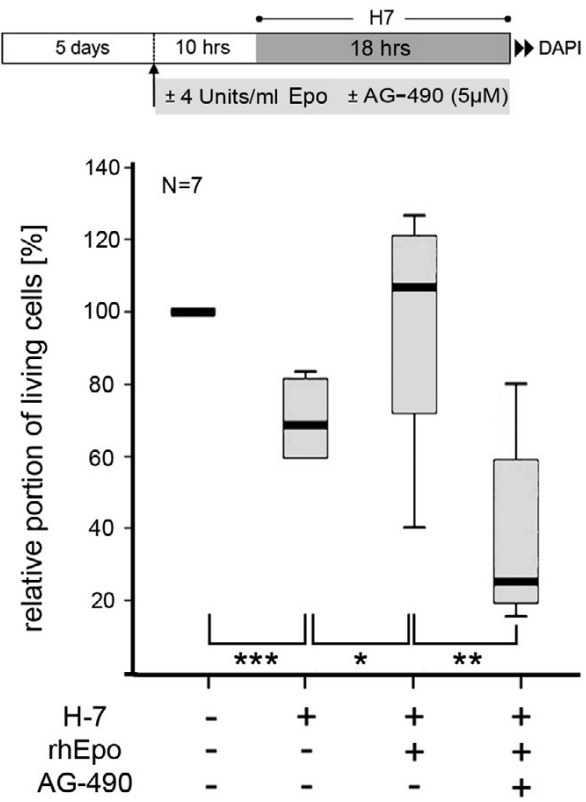

Figure 8. RhEpo supports survival of locust neu rons exposed to apoptosis-inducing stimuli via AG-490-sensitive Janus kinase activity. (A) Apoptosis induced by hypoxia was completely prevented by pretreatment with $4 \mathrm{U} / \mathrm{ml}$ rhEpo $\left.{ }^{* * *} P<0.001\right)$, while the protective effects of rhEpo was blocked by $5 \mu$ M AG-490 $\left.{ }^{* * *} P<0.001\right) . N=13$. (B) Similarly, H-7 induced apoptosis was prevented by rhEpo $\left({ }^{*} P<0.05\right)$, but not when AG490 $(5 \mu \mathrm{M})$ was incubated together with rhEpo $\left({ }^{* *} P<0.01\right)$. $N=7$. Statistics: Kruskal-Wallis ANOVA, Mann-Whitney $U$-test with Benjamini-Hochberg-correction. ${ }^{*} P<0.05,{ }^{* *} P<0.01,{ }^{* * *} P<0.001$. Schematics in the upper parts indicate experimental procedures.

Once activated, JAK2 phosphorylates intracellular tyrosine residues of EpoR that serve as docking sites for intracellular signaling proteins with Src homology 2 (SH2) domains. Upon their binding to the phosphotyrosyl residues 
of EpoR, these proteins are phosphorylated by JAK2. Phosphorylation of STAT5 by JAK2, which allows STAT5 dimerization and its activation as transcription factor, was demonstrated to be essential for Epo-mediated erythropoietic and tissue-protective activity (Sirén et al., 2001; Chateauvieux et al. 2011). To investigate a potential involvement of STAT proteins in locust neuroprotection, the membrane permeable STAT inhibitor sc-355979 was used.

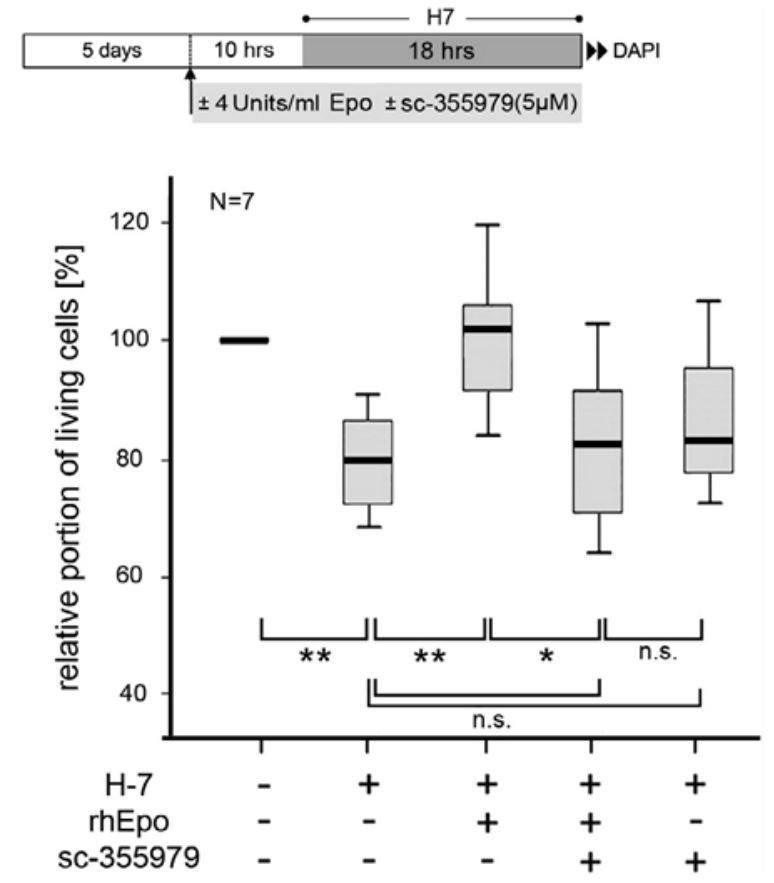

Figure 9. Activation of sc-355979-sensitive STAT phosphorylation is required for the antiapoptotic effects of rhEpo in primary cultures of locust neurons. H-7 induced apoptosis $\left({ }^{* *} P<0.01\right)$ was efficiently suppressed by $4 \mathrm{U} / \mathrm{ml}$ rhEpo $\left({ }^{* *} P<0.01\right)$. Co-application of $5 \mu \mathrm{M}$ sc355979 prevented the antiapoptotic effect of rhEpo $\left({ }^{*} P<0.05\right)$. N=7. Statistics: Kruskal-Wallis ANOVA, Mann-Whitney $U$-test with Benjamini-Hochberg-correction. ${ }^{*} P<0.05, \quad{ }^{*} P<0.01$. Experimental timeline is illustrated in the upper part of the figure.

Apoptosis-induced by H-7 reduced neuronal survival (median of surviving neurons: $79.83 \%)$ compared to untreated controls $(P=0.0058)$, while rhEpo fully protected locust neurons from H-7-induced apoptotic cell death (median: $101.98 \%$, difference to H-7-treated group $P=0.00699$ ) (Fig. 9). This neuroprotective effect of rhEpo against H-7-induced apoptosis was completely blocked by simultaneous incubation of neuronal cultures with sc-355979 
(median: $82.79 \%, P=0.0483$ ), leading to a reduction of neuronal survival to a level that did not differ from survival of neuronal cultures treated with H-7 only $(P=0.5349)$. Co-incubation of neuronal cultures with H-7 and sc-355979 did not cause an additional reduction of neuronal survival compared to $\mathrm{H}-7$ treated cultures (median: 83.52\%, $P=0.5349$ ). This suggested that sc-355979 predominantly interfered with protective pathways initiated by rhEpo rather than reducing cell viability by a different mechanism not related to Epo. Therefore, the neuroprotective effect of Epo on H-7-treated locust neurons seems to be transduced via the activation of STAT transcription factors.

Taken together, these results suggest that the JAK/STAT signaling pathway is critical for the antiapoptotic activity of rhEpo signaling in locust neurons in vitro.

\section{I.3.4 Epo-mediated neuroprotection does not require PI3K and NF-KB activity}

One of the signaling molecules with a SH2 domain that is attracted by the phosphotyrosyl residues of the cytoplasmic domain of EpoR is the regulatory subunit of PI3K. The PI3K pathway regulates apoptosis in various tissues through phosphorylation of its cellular target Akt. The ATP-binding site of PI3K can be blocked with LY294002, preventing the PI3K-mediated activation of Akt (Vlahos et al. 1994). As shown in Figure 10A, the capability of rhEpo to protect locust neurons against H-7 induced apoptosis was not compromised by application of $5 \mu \mathrm{M}$ LY294002. RhEpo increased survival of neurons exposed to H-7 in both conditions, when applied alone (median: $105.08 \%, P=0.0022$ ) and upon co-application with LY294002 (median: 100.69\%, P=0.0303), and there was no difference in neuronal survival between these two groups $(P=0.3939)$. Survival of neurons exposed to H-7 (median: 72.94\%) was not further reduced by incubation with $5 \mu \mathrm{M}$ LY294002 (median: 77.02\%, $P=0.3095$ ), indicating that LY294002 had no negative effect on neuronal survival. To confirm these results, the ability of LY294002 to affect the neuroprotective action of rhEpo was tested 
in primary cultures of locust neurons exposed to hypoxia (Fig. 10B). Like in H-7 treated cultures, rhEpo-mediated neuroprotection against hypoxia-induced cell death persisted in the presence of LY294002 (median: 89.54\%), and did not differ from rhEpo-mediated protection of locust neurons exposed to hypoxia (median: 94.13\%, $P=0.3095)$. Neuronal survival in cultures threated with LY294002 and exposed to hypoxia (median: 64.08\%) did not differ from the survival of neurons subjected to hypoxia only (median: 61.12\%, $P=0.5476$ ), indicating that $5 \mu \mathrm{M}$ LY294002 had no detrimental effect on neuronal viability.

A
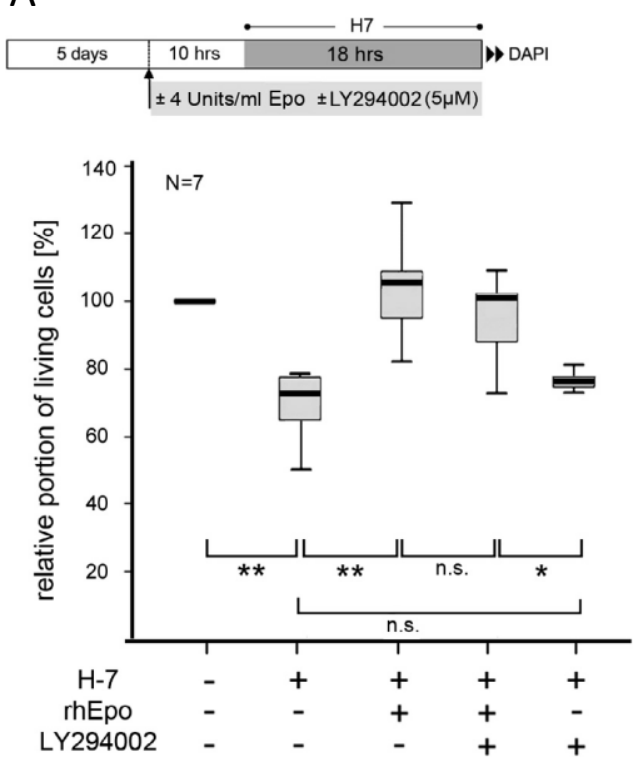

B
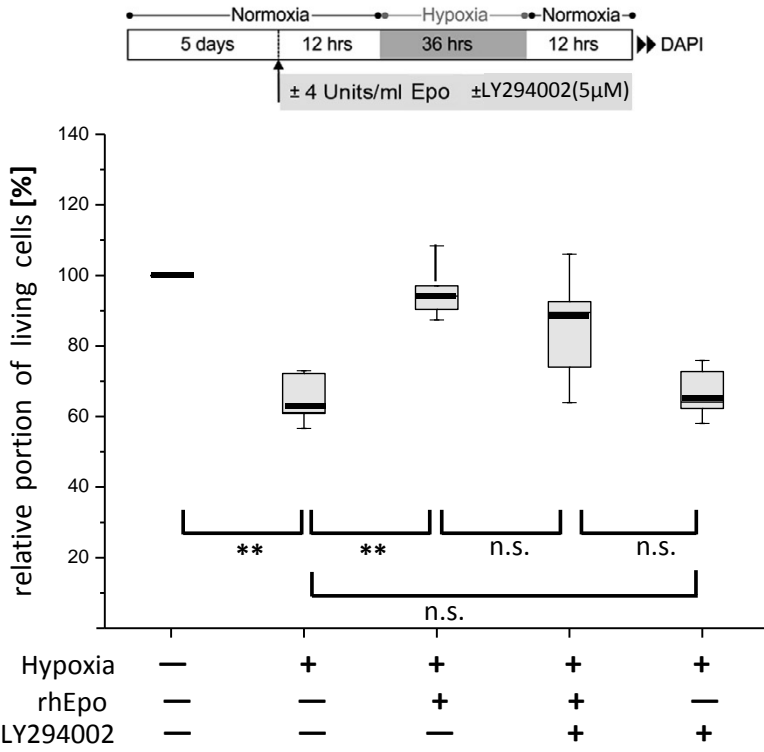

Figure 10. RhEpo-mediated protection of primary cultured locust brain neurons is independent of LY294002-sensitive PI3K activity. Both, H-7- $\left.{ }^{* *} P<0.01\right)$ and hypoxia- $\left({ }^{* *} P<0.01\right)$ induced apoptosis were prevented by $4 \mathrm{U} / \mathrm{ml}$ rhEpo $\left({ }^{* *} P<0.01, \mathbf{A}, \mathbf{B}\right)$, and application of $5 \mu \mathrm{M} L Y 294002$ did not prevent neuroprotective effects of rhEpo (hypoxia: n.s.; H-7: n.s.). LY294002 was added to primary cultured locust neurons either at the same time as rhEpo ( $\mathrm{H}-7$ experiments, $N=7, \mathbf{A})$ or $2 \mathrm{~h}$ before application of rhEpo (hypoxia experiments, $N=5$, B). Statistics: Kruskal-Wallis ANOVA, Mann-Whitney $U$-test with Benjamini-Hochberg-correction. ${ }^{*} P<0.05, \quad{ }^{* *} P<0.01$. Experimental procedure is schematically illustrated in the upper part of the figure.

However, when applied in higher concentrations $(50 \mu \mathrm{M}$ and $100 \mu \mathrm{M})$, LY294002 reduced neuronal survival in primary cell cultures from locust brains (Fig. 11). This suggests (assuming a specific action of LY294002 on PI3K) that a certain basal activity of PI3K/ Akt signaling is required for cell survival. 


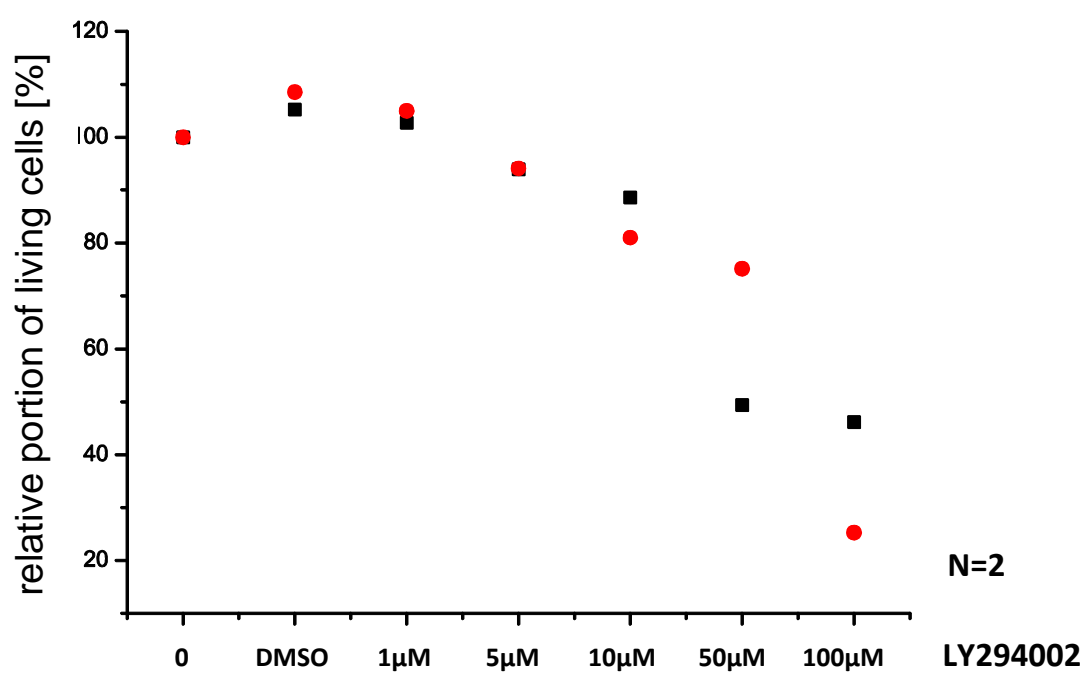

Figure 11. Effects of different concentrations of LY294002 on the survival of primary cultured locust brain neurons. Cultures were exposed to LY294002 for 60 hours. Concentration of DMSO in culture media was $1 \%$ and corresponds to the concentration of DMSO in the cell cultures treated with $100 \mu \mathrm{M}$ LY294002. N=2.

Digicaylioglu and Lipton demonstrated that EpoR-mediated activation of JAK2 in mammalian neurons leads to phosphorylation of the inhibitor of NF-kB (ІКB), which leads to the release of NF-KB and its translocation into the nucleus where it activates transcription of anti-apoptotic genes (Digicaylioglu \& Lipton 2001). To investigate a potential contribution of NF-kB signaling to rhEpo-mediated protection of locust neurons, we used pyrrolidine dithiocarbamate (PDTC) to block the dissociation of ІкB from the cytoplasmic NF-кB dimer. As in the previously described experiments, hypoxia reduced the survival of locust neurons (mean of neuronal survival: 71.11\%), while rhEpo protected neurons from hypoxia-induced apoptosis (mean: 94.57\%) (Fig. 12). This neuroprotective effect of rhEpo was not abolished by simultaneous incubation with PDTC (mean: 92.45\%). Unexpectedly, PDTC alone partially reduced pro-apoptotic effects of hypoxia (mean: $83.89 \%$ ). This anti-apoptotic action of PDTC was not observed in locust neurons cultured under normoxic conditions (mean: 88.55\%). 

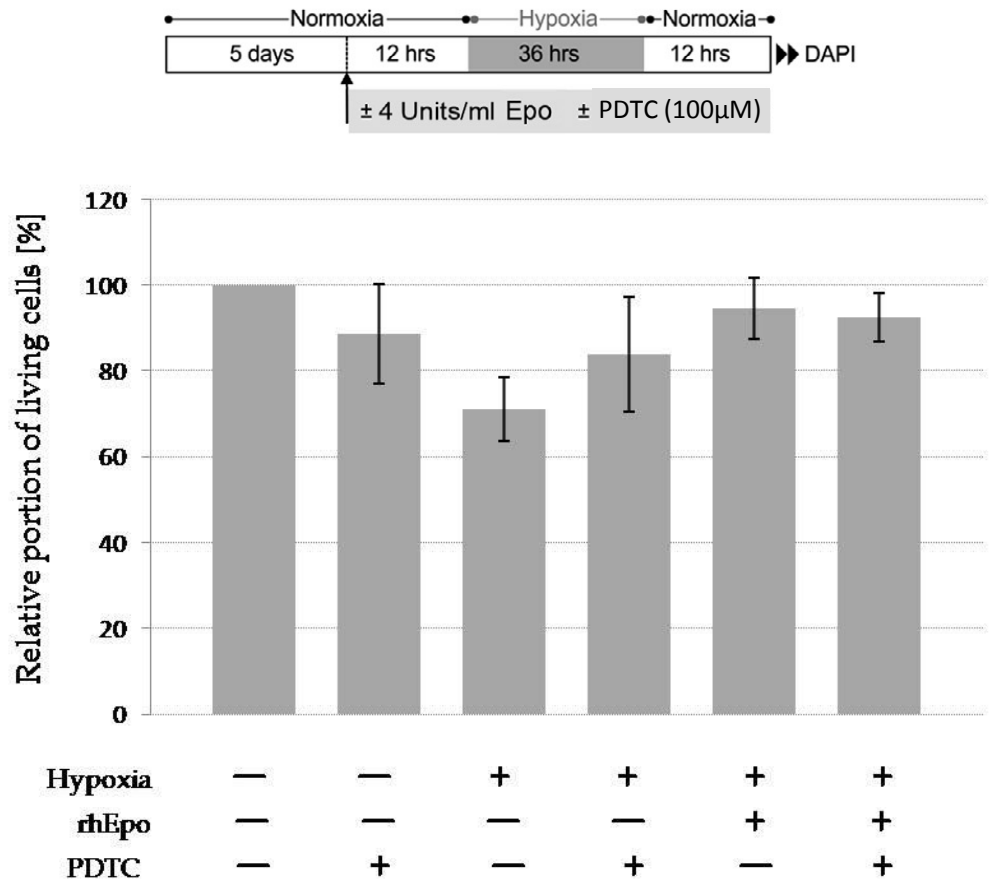

Figure 12. RhEpo-mediated neuroprotection of locust neurons is independent of PDTCsensitive IкB/NF-kB activity. RhEpo $(4 \mathrm{U} / \mathrm{ml})$ applied $12 \mathrm{~h}$ before onset of hypoxia prevented hypoxia-induced apop tosis. PDTC $(100 \mu \mathrm{M})$ partially supressed hypoxia-induced apoptosis and did not abolish neuroprotective effects of rhEpo. PDTC was applied $2 \mathrm{~h}$ before application of rhEpo. Each bar represents mean \pm SED. $N=3$. No statistics. Schematic of experimental procedure is shown in the upper part of the figure.

Altogether, these results suggest that rhEpo may not use PI3K/ Akt and NF-kB pathways to suppress apoptosis and promote protection of locust brain neurons in vitro. 


\section{I.4 Discussion}

\section{I.4.1 Epo-mediated protection of locust brain neurons from hypoxia- and H-7 induced apoptosis}

Epo-mediated neuroprotection following exposure to a variety of mechanical and physiological insults has been documented in a number of in vivo and in vitro studies on mammalian nervous systems. Epo protects mammalian neurons from hypoxia, ischemic brain injury, inflammation, oxidative stress, metabolic stress, glutamate toxicity (Morishita et al. 1997; Bernaudin et al. 1999; Lewczuk et al. 2000; Sirén et al. 2001a; Chong et al. 2003) and other damaging conditions. The protective effects of Epo are accomplished by prevention of neuronal apoptosis and promotion of cell survival. The programmed cell death apoptosis consists of a highly regulated series of events that lead to the cleavage of genomic DNA, membrane blebbing, cell shrinkage and fragmentation into membrane-enclosed vesicles called apoptotic bodies. These irreversible cellular changes are initiated by the proteolytic activity of the conserved family of cysteinyl aspartate-specific proteases, the caspases (Green 1998; Cooper et al. 2009). The core components and molecular mechanisms of the cell death machinery are conserved through evolution, from worms to mammals, including insects (Richardson \& Kumar 2002). Seven caspases (three initiator and four effector caspases) have been identified in Drosophila melanogaster. Drosophila Dredd, Dronc and Strica are homologues of mammalian caspase-8, 9, -10 that function as initiator caspases (Cooper et al. 2009). In mammalian cells, activation of caspase- 9 is stimulated by Apaf- 1 upon the release of cytochrome c from mitochondria into the cytoplasm. In fruit flies, Dark (Drosophila Apaf-1-related killer) is the functional orthologue of mammalian Apaf-1 required for Dronc activation (Rodriguez et al. 1999). Following the transmission of a cell death signal, the initiator caspases proteolitically activate downstream effector caspases, which then cleave the substrates responsible for the dismantling of the cell. In mammals, the effector caspases are caspase-3, -6 and -7, and respective homologues in Drosophila are Drice, Dcp-1, Decay and Damm (Manji \& Friesen 2001; Cooper et al. 2009). In insects, apoptosis plays a 
critical role in development, tissue homeostasis and defense against pathogens (Bergmann et al. 1998).

One of the two stimuli that have been used to induce apoptosis of primary cultured locust brain neurons was exposure to hypoxic conditions for 36 hours. Hypoxia is a condition of limited availability of oxygen to the cells. The optimal functioning of most cells is tightly linked to the availability and usage of oxygen as an acceptor of electrons during oxidative phosphorylation to generate ATP (Loiacono \& Shapiro 2010). Hence, the ability to sense and adapt cellular metabolism to changes in oxygen levels is essential for the functioning of tissues and the survival of an organism. In order to maintain homeostasis cells and tissues have developed different oxygen-sensing mechanisms and various strategies to adapt to reduced environmental oxygen levels (Giaccia et al. 2004). The ability of an organism to withstand hypoxia critically depends on the sensitivity of its nervous system to an interrupted oxygen supply. While mammalian neurons are extremely vulnerable to ischemia and in the absence of oxygen undergo irreversible damage leading to cell death in just a few minutes (Dowling 2007), some insects exhibit a remarkable tolerance to oxygen deprivation (Hoback \& Stanley 2001). Cockroaches (Periplaneta americana) are able to survive several hours under anoxia, recovering fully upon returning to normoxic conditions (Pitman 1988). Like cockroaches, fruit flies (Drosophila melanogaster) are extremely tolerant to oxygen deprivation and after a period of exposure to levels and durations of hypoxia that induce irreversible injury and death in rodents the brain of fruit flies does not suffer any damage (Zhao \& Haddad 2011). The natural habitats of locusts (Locusta migratoria) are semi-arid regions of the world (Scanlan et al. 2001) including regions of Africa that are periodically flooded (Rainey 1951). Adult locusts can survive several hours of anoxia that results from suffocation during the flooding (Hochachka et al. 1993; Hoback \& Stanley 2001; Greenlee \& Harrison 2004; Brust et al. 2007).

At least some of the mechanisms underlying hypoxia-tolerance seem to be conserved across invertebrate and vertebrate species. The hypoxic responses are 
primarily mediated by a family of highly conserved transcription factors named hypoxia inducible factors (HIFs) (Gorr et al. 2006). HIF forms heterodimers of aand $\beta$-subunits (Wang \& Semenza 1995). In response to low oxygen partial pressure HIF specifically binds target gene sequences, so-called hypoxiaresponse elements (HREs) (Firth et al. 1995), inducing transcription of genes that mediate adaptation to oxygen deprivation such as the epo gene (Maxwell et al. 1993). Homologs of $\alpha$ and $\beta$ HIF subunits have been reported in $D$. melanogaster, in which Sima is the fly orthologue of mammalian HIF-a (Nambu et al. 1996) and Tango is the fly orthologue of HIF- $\beta$ (Lavista-Llanos et al. 2002; Dekanty et al. 2010). To withstand oxygen deprivation insects and mammals employ notably different strategies that are governed by HIF. In insects, HIFdriven regulation of target genes mediates adaptation to hypoxia by directing the cellular metabolism to energy conservation mode, rather than energy compensation mode, which prevails in mammals (Gorr et al. 2006). To conserve energy insect cells are able to reversibly enter a state of a regular metabolic depression, characterized by a drastically reduced consumption of ATP (Gorr et al. 2006).

The second approach that has been used to induce apoptosis in primary cultured locust brain neurons was treatment with the chemical H-7. Though H7 has been shown to inhibit cAMP-dependent protein kinase, protein kinase $C$, cGMP-dependent protein kinase, myosin light chain kinase, and casein kinase I, studies on a Drosophila neuronal cell line (Nagano et al. 1998) and on human neuroblastoma cells reported that H-7 induces apoptosis by unidentified mechanisms that are independent of protein kinase inhibition (Ronca et al. 1997). Moreover, H-7 seems to initiate apoptosis through cell type-specific targets, since Schneider cells were not affected by H-7 incubation (Nagano et al. 1998). In addition, the structure and biological activities of H-7 have been shown to differ depending on their commercial supplier (Quick et al. 1992), which might partially explain the discrepancies between observations obtained from independent studies. Similarly to Drosophila neuronal cell cultures, H-7 has induced cell death in primary cultures of locust brain neurons that 
displayed typical apoptotic features including the activation of caspase-3, DNA fragmentation, nuclear condensation and the ultimate disintegration of cytoplasmic membranes, leaving nuclear remnants (pyknotic bodies) (Kroemer et al. 2009; Miljus et al. 2014). Both, hypoxia- and H-7-induced apoptosis of locust neurons was efficiently prevented by rhEpo. This suggests that Epo interfered with certain components of the apoptotic cascade to counteract cell death.

\section{I.4.2 Requirement of JAK/STAT signaling for Epo-mediated protection of locust brain neurons}

Epo mediated neuroprotection in mammals is governed by transduction pathways that have similarities to, as well as differences from, those operating during erythropoiesis. Both, neuroprotection and erythropoiesis are initiated by receptor-associated JAK2 tyrosine kinases that transduce signals from activated receptors to downstream cellular effectors. Hence, binding of Epo to either classical homodimeric EpoR or heterodimeric EpoR induces a conformational change in the receptor (Remy et al. 1999) that brings receptor associated JAK2 molecules into close proximity enabling their reciprocal interaction and activation by trans-phosphorylation (Witthuhn et al. 1993). Once activated, JAK2 phosphorylates specific tyrosine residues on the cytoplasmic domain of the receptor, creating docking sites for signaling molecules with $\mathrm{SH} 2$ domains. The best known effector molecules (with $\mathrm{SH} 2$ domain) that are recruited to phosphotyrosyl residues of EpoR and phosphorylated by JAK2 are the members of the signal transducer and activator of transcription (STAT) family (Yamaoka et al. 2004). Especially STAT5 and STAT3 have been implicated in Epo-stimulated erythropoiesis and protective mechanisms in various nonhematopoietic mammalian tissues including the nervous system (Sirén et al. 2001a; Chateauvieux et al. 2011). Phosphorylated STATs reorient into antiparallel dimers, in which the phosho-tyrosine residue of one STAT binds the SH2 domain of the other STAT (Schindler et al. 2007). STAT dimers 
activated in this manner translocate to the nucleus where they bind specific enhancer elements inducing transcription of anti-apoptotic genes.

In mammals four members of the JAK and seven members of the STAT family have been described (Schindler et al. 2007). In the fruit fly a single JAK homologue, Hopscotch (Perrimon \& Mahowald 1986; Binari \& Perrimon 1994) and a single STAT homologue STAT94E (Hou et al. 1996; Yan et al. 1996) have been identified. Drosphila's Hopscotch is most similar to mammalian JAK1 and JAK2, while STAT92E is most similar to mammalian STAT3 and STAT5. The canonical JAK/STAT signaling pathway in Drosophila is activated by three related ligands called Unpaired (Harrison et al. 1998; Boulay et al. 2003), Unpaired 2 (Hombría et al. 2005) and Unpaired 3 (Agaisse et al. 2003) that bind to the Domeless receptor (Brown et al. 2001). According to Wang and colleagues (Wang et al. 2014) genes coding for six orthologues of JAK/STAT pathway members have been identified in Locusta migratoria genome. The JAK/STAT pathway in Drosophila is required for embryonic segmentation, tracheal development, larval hematopoiesis, stem cell maintenance (Bina \& Zeidler 2009) and innate immune responses to injury (Agaisse et al. 2003).

JAK2 is sensitive to the membrane permeable tyrphostin AG-490. AG-490 has been used to block both mammalian JAK2- (Digicaylioglu \& Lipton 2001; Witthuhn et al. 1993) and insect JAK-initiated signaling cascades (Hamshou et al. 2012). We demonstrated that AG-490 completely abolished the neuroprotective effects of rhEpo on primary cultured locust brain neurons in the presence of two different apoptotic stimuli. This indicates the presence of a receptor that, similar to the mammalian hematopoietic and non-hematopoietic EpoR, initiates anti-apoptotic mechanisms through JAK-mediated phosphorylation of intracellular domains.

The membrane permeable compound sc-355979 binds to the SH2 domain of STAT and prevents its activation through association with the receptor/JAK complex. Sc-355979 has been demonstrated to suppress Epo-initiated STAT5 activation in mammals (Tramontano et al. 2003; Kittilson et al. 2011; Dieudonne 
et al. 2013). The STAT inhibitor also suppressed rhEpo-mediated neuroprotection during H-7-induced apoptosis in locust brain neurons, indicating that Epo regulates the expression of beneficial genes via JAK/STAT signaling in both mammals and insects.

\section{I.4.3 Contribution of PI3K and NF-KB pathways to Epo-mediated protection of locust brain neurons}

PI3K/ Akt signaling has been identified as the major transduction pathway for Epo-mediated cell protection in various mammalian non-hematopoietic tissues, including the nervous system (Sirén et al. 2001a; Ruscher et al. 2002; Chong \& Maiese 2005; Chateauvieux et al. 2011). PI3Ks are heterodimers composed of a regulatory subunit P85 and a catalytic subunit P110 (Cantrell 2001). The regulatory subunit P85 binds to phosphorylated tyrosine residues of the activated EpoR via its SH2 domain (Witthuhn et al. 1993). Once bound, it recruits the catalytic subunit P110 to form the fully active PI3K enzyme. Activated PI3K converts plasma membrane phosphoinositides to phosphatidylinositols, which serve as membrane docking sites for proteins with pleckstrin-homology $(\mathrm{PH})$ domains. An important downstream effector activated by the PI3-kinase signaling pathway is the serine/threonine kinase Akt, also known as protein kinase B (Zhao et al. 2006). Akt is recruited to phosphatidylinositol-rich membranes via its $\mathrm{PH}$ domain. At the membrane, Akt is activated after being phosphorylated at two positions by two distinct kinases, phosphatidylinositol dependent kinase 1 (at Thr308) and mammalian target of rapamycin complex 2 (mTORC2) (at Ser474) (Bao et al. 1999). Once active, Akt translocates from the plasma membrane to the cytosol, where it modulates the function of key regulatory proteins and enzymes by phosphorylation. In the nucleus Akt inhibits transcription factors that promote the expression of cell death genes and enhances transcription of anti-apoptotic genes.

The PI3K/Akt signaling pathway is conserved among eukaryotic organisms (Scanga et al. 2000). Drosophila PI3K (dPI3K) (Leevers et al. 1996) and Drosophila 
Akt (d Akt) (Franke et al. 1994) have been shown to be involved in various physiological responses in flies including anti-apoptotic mechanisms (Staveley et al. 1998). Genes coding for PI3K (LOCMI07824) and Akt (LOCMI16427) have also been identified in the genome of Locusta migratoria (Wang et al. 2014).

The membrane permeable compound LY294002 has been described to act as a specific inhibitor of PI3K (Vlahos et al. 1994). LY294002 has been used previously to prevent the PI3K-induced phosphorylation of Akt and abolish the protective effects of Epo in mammals (Nakazawa et al. 2003; Trincavelli et al. 2013). Several studies on insects successfully employed LY294002 to suppress PI3K signaling (Lizcano et al. 2003; Kim et al. 2004; Mounir et al. 2011; Gu et al. 2012; Roy \& Raikhel 2012), indicating that the compound also effectively inhibits insect orthologues of PI3K. In our study, co-application of LY294002 did not reduce the neuroprotective effects of Epo on hypoxia-exposed and H-7treated locust brain neurons, suggesting that no PI3K homolog contributes to Epo-mediated protection of locust neurons. Since higher concentrations of LY294002 (10 $\mu \mathrm{M}$ or more) reduced the viability of locust brain neurons under normal conditions, LY294002-sensitive PI3K seems to be present and its activity required for cellular survival. Although PI3K is the major mode of Akt activation, Akt can also be activated directly by other tyrosine or serine/threonine kinases, even when PI3K activity is inhibited by LY294002 (Mahajan \& Mahajan 2013). Similarly, one study in insects reported that LY294002 was not potent at inhibiting dAkt activation (Lizcano et al. 2003). In addition, LY294002 may also inhibit the activity of other kinases, such as mTOR (Brunn et al. 1996) and dTOR (Lizcano et al. 2003), with similar potency as on PI3K.

The NF-kB pathway has been reported to play an important role in Epomediated protection of mammalian neurons (Digicaylioglu \& Lipton 2001; Liu et al. 2005) but does not contribute to Epo-mediated effects in the hematopoietic system. NF-kB (nuclear factor kappa-light-chain-enhancer of activated B cells) proteins comprise a family of structurally related and evolutionarily conserved 
transcription factors that regulate the expression of genes involved in cellular responses to infection, stress and injury (Ghosh et al. 1998). In mammals five members of NF-kB proteins associate with each other to form various transcriptionally active homo- and hetero-dimeric complexes (Hoffmann \& Baltimore 2006; Manavalan et al. 2010). In unstimulated cells, the NF-kB dimers are bound and inhibited by inhibitors of кappa B proteins (IкBs) (Baeuerle \& Baltimore 1988). By virtue of their ankyrin repeat domains, the IkB proteins mask the nuclear localization signals of NF-kB proteins and keep them sequestered in an inactive state in the cytoplasm (Jacobs \& Harrison 1998). Signals arising from external ligands lead to the activation of the IкB kinase (IKK) that phosphorylates the IKB protein. Phosphorylation of IKB leads to its rapid ubiquitination and proteasomal degradation, resulting in its dissociation from the NF-kB dimer. The liberated NF- $\mathrm{kB}$ dimer then translocates to the nucleus and induces transcription of target genes, such as cytokine genes, stress response genes, pro-survival genes, anti-apoptotic genes, antioxidant enzyme genes and other transcription factors (over 150 target genes in vertebrates) (Pahl 1999). NF-kB positively regulates the expression of its own repressor, IкB. The newly synthesized IкB then re-inhibits NF-kB forming thus an auto feedback loop, which results in oscillating levels of NF-KB activity (Nelson 2004).

All five members of the NF-kB family described in mammals share a conservative Rel homology (RH) domain, responsible for DNA-binding, dimerization and interaction with IkB (Ghosh et al. 1998). Dif, Dorsal and Relish are Drosophila NF-kB homologues (Steward 1987; Ip et al. 1993; Dushay et al. 1996) with conserved RH domains contained in their structures (Minakhina \& Steward 2006). Dif and Dorsal are retained in the cytoplasm of resting cells by Cactus, the Drosophila homolog of mammalian IkB proteins (Tanji \& Ip 2005). Degradation of Cactus, which is mediated by Drosophila kinase Pelle in an as yet undefined manner (Towb et al. 2001; Huang et al. 2010), releases Dif and Dorsal that subsequently translocate to the nucleus and activate the expression of antimicrobial peptide genes (Tanji \& Ip 2005). The third NF-kB Drosophila homologue, Relish, in addition to its N-terminal RH domain contains a C- 
terminal IKB-like region (Basith et al. 2013). The Drosophila IKK complex directs the site-specific proteolytic cleavage and activation of Relish (Stöven et al. 2000; Stöven et al. 2003). In addition to flies, orthologues of NF-kB are found in a number of other insects (Minakhina \& Steward 2006), such as mosquitoes (Waterhouse et al. 2007), moths (Sun \& Faye 1992), beetles (Sagisaka et al. 2004), and locusts, whose genome contains genes that code for three Rel like NF-kB proteins (Wang et al. 2014). The ancient origin of NF-kB proteins is supported by their presence in the most archaic arthropod, the horseshoe crab Limulus (Wang et al. 2006a).

The requirement of NF-kB for Epo-mediated protection of rat cerebrocortical neurons has been investigated using the membrane permeable compound pyrrolidine dithiocarbamate (PDTC) to inhibit NF-kB activation (Digicaylioglu \& Lipton 2001). PDTC has also been used to block the activity of insect orthologues of NF-kB (Luo et al. 2013). The mechanism through which PDTC inhibits NF-kB involves the suppression of IкB release from the cytoplasmic form of NF-kB through the interaction with reactive oxygen species (ROS). This ability of PDTC presumably relies on its chelating and antioxidative properties (Schreck et al. 1992). Application of PDTC two hours before introducing rhEpo did not suppress neuroprotective effects of rhEpo on hypoxia-exposed locust brain neurons, indicating that the NF-KB pathway may not be implicated in Epo-mediated protection of locust neurons. As application of PDTC alone on otherwise non-treated locust brain neurons reduced their survival, the activity of locust NF-kB orthologues has been most likely suppressed and its basal activity seems to be required for cellular survival. Even though it seems that application of PDTC alone under hypoxic condition increased the viability of locust brain neurons, this effect may not be significant, since there is a high variation between individual experiments. Due to the low number of experimental trials, the significance levels were not statistically analyzed. Nevertheless, it should be noted that intracellular levels of ROS play an important role in regulating the activity of NF-kB (Morgan \& Liu 2011). As mentioned above, it is believed that the thiol-containing compound PDTC 
prevents the dissociation if $\mathrm{I} \kappa \mathrm{B}$ from $\mathrm{NF}-\mathrm{\kappa} B$ and subsequent activation of the NF-kB transduction cascade by scavenging ROS (Schreck et al. 1992). Hypoxia may lead to increased generation of ROS (Chandel et al. 2000). Under these conditions scavenging of ROS by PDTC might have contributed to both, suppression of NF-kB activation and reduction of cellular damage induced by ROS. Indeed, some reports suggested that PDTC can act as an anti-oxidant that promotes cellular survival (Moellering et al. 1999; Lee et al. 1999; La Rosa et al. 2004). Protective effects of PDTC against brain ischemia have been also reported (Nurmi et al. 2004). However, it is more likely that PDTC is affecting the overall cellular balance between oxidants and antioxidants (Haddad et al. 2000). The ultimate consequence of cell injury hence depends on the level at which the cell's antioxidative defense mechanisms are compromised by hypoxic stress. Whether the NF-kB pathway will promote neuronal death or survival depends as well on the cell type, the timing of the NF-kB activation (Pizzi et al. 2002) and the type of NF-kB dimers that are activated (Mattson \& Meffert 2006; Sarnico et al. 2009). An alternative approach to study the requirement of NF-kB for Epomediated neuroprotection can be for instance the determination of cytoplasmic and nuclear levels of NF-kB. If Epo uses the NF-kB pathway to transmit prosurvival signals nuclear translocation of NF-kB would be expected (Digicaylioglu \& Lipton 2001; Shingo et al. 2001).

Finally, the exact nature of the NF-kB response to specific stimuli depends on the cross-talk between different transduction pathways and their integration with other cellular processes (Manning \& Cantley 2007). NF-kB signaling can be modulated for instance by Akt that can phosphorylate and activate IKK to allow expression of pro-survival genes (Romashkova \& Makarov 1999; Chong et al. 2005). In specific types of cells, STAT5 also plays a role in regulating the NF-кB signaling pathway (Nakamura et al. 2002). Since Akt can be activated even when the activity of PI3K is blocked by LY294002 (Mahajan \& Mahajan 2013), a certain level of Akt and NF-kB involvement in Epo-mediated protection of locust brain neurons cannot be completely excluded. 
Taken together, the experimental results described in this chapter document that Epo-mediated protection, comparable to that known for mammalian cells, can also be observed in insect neurons subjected to apoptotic stimuli. Moreover, pathways that are critical for Epo protection of locust brain neurons are partly identical with the pathways involved in Epo effects on mammalian neurons. The results demonstrate the requirement of JAK and STAT, but not necessarily of PI3K and NF-KB for Epo-mediated beneficial mechanisms that interfere with apoptotic processes. The effects reported in this study suggest the presence of a receptor with ligand binding properties that enable its activation by rhEpo followed by the activation of JAK/STAT signaling to initiate anti-apoptotic cellular responses. These results support the hypothesis of a phylogenetically old role for Epo-like signaling in tissue protection that was already present in the last common ancestor of insects and vertebrates. 


\section{Studies on the receptor that mediates neuroprotective properties of erythropoietin in Locusta migratoria}

\section{II.1 Introduction}

Both hematopoietic and non-hematopoietic effects of Epo in mammals are mediated through its binding to a cell surface membrane receptor. Hematopoietic EpoR is a member of the type I superfamily of singletransmembrane chain cytokine receptors. These receptors share several common structural motifs: an N-terminal domain with conserved fibronectin III-like subdomains, a WSXWS motif with conserved cysteines that is important for protein folding; a single hydrophobic transmembrane segment; and a cytoplasmic domain with conserved regions termed Box1 and Box2 that associate with members of the Janus kinase family. Type I cytokine receptors may bind several ligands and trigger intracellular signaling events as homodimers (Youssoufian et al. 1993; Boulay et al. 2003). EpoR is expressed on the cell surface as a pre-formed receptor homodimer (Livnah et al. 1999) connected by the leucine zipper of the transmembrane domains (Constantinescu et al. 2001). The abundance of EpoR on the surface of erythroid progenitor cells is very low, with approximately one thousand receptors present per cell (Youssoufian et al. 1993; Lacombe \& Mayeux 1998) while the majority of receptor proteins reside in intracellular pools (Yoshimura et al. 1990). Non-hematopoietic cell types have been demonstrated to express even lower numbers of functional receptors in their cytomembranes (Um \& Lodish 2005; Becker et al. 2010; Elliott et al. 2014). Gross and Lodish (2006) showed that upon binding to hematopoietic EpoR, Epo is internalized via receptor-mediated endocytosis and subsequently degraded in lysosomes. Receptor-mediated endocytosis in bone marrow, liver and kidney contributes to the clearance of circulating Epo from the serum (Jelkmann 2002). The rates of intracellular Epo degradation are determined by binding, dissociation and trafficking properties of its receptor (Gross \& Lodish 2006). It remains, however, unclear whether or 
not Epo is internalized and degraded in the same way by Epo-responsive nonhematopoietic cells, such as neurons.

Even though hematopoietic EpoR is clearly expressed in various cell types of the mammalian nervous system, including neurons, astrocytes, microglia, oligodendrocyte progenitor cells, and endothelial cells (Digicaylioglu et al. 1995; Marti et al. 1996; Liu et al. 1997; Nagai et al. 2001; Sugawa et al. 2002; Ott et al. 2015) and upregulated during ischemia/hypoxia (Sakanaka et al. 1998; Sirén et al. 2001b; Ott et al. 2015), whether or not it is involved in conveying the neuroprotective properties of Epo is still controversial. Some studies have shown that affinities of Epo binding sites on PC12 rat neuronal cells (Masuda et al. 1993) and brain endothelial cells (Yamaji et al. 1996) are much lower than those on erythroid cells. Moreover, it has been shown that brain-derived Epo has less sialic groups (Masuda et al. 1994). These observations indicated that the endogenous Epo/EpoR system mediating tissue protective properties might have a distinct bioactivity and regulatory system that differs from the erythropoietic Epo/EpoR system. Further studies showed that different Epo derivatives made by chemical modifications of rhEpo, such as asialo-Epo (Erbayraktar et al. 2003), carbamylated Epo (cEpo) (Leist et al. 2004), Epobis (Pankratova et al. 2012), ARA290 - an 11 amino acid peptide derived from a tertiary structure of Epo- (Brines et al. 2008) exerted neuroprotection, without stimulating erythropoiesis. This substantiated the idea that neuroprotective effects of Epo and Epo derivatives could be mediated through a specific receptor. The enigmatic tissue-protective receptor has been postulated to be a heterodimeric receptor complex consisting of a monomer of classical EpoR and another class I cytokine receptor monomer, for instance beta common $(\beta c)$ chain (Brines et al. 2004). However, Epo-mediated neuroprotection of rat hippocampal neurons (Nadam et al. 2007) and a neuron-like cell line (Um \& Lodish 2005) was accomplished in the absence of functional $\beta$ common chain receptor. Thus, if EpoR forms a functional tissue-protective receptor by association with $\beta c$ chain, it may only be the case in particular cell types and/or under particular conditions. Ephrine B4 receptor has been proposed as another 
partner of the EpoR monomer to form a heteromeric tissue-protective receptor (Jackson et al. 2012; Debeljak et al. 2014). The alternative receptor that specifically mediates tissue protection could also be a heterodimer or homomer formed by yet unidentified subunits.

Recently a splice variant of the human epo gene that lacks intron 3 of full-length epo has been identified (Bonnas 2009). The recombinant human protein EV3 had no stimulatory effect on mouse erythropoiesis but was similarly neuroprotective as rhEpo in primary cultures of rat cortex neurons that were challenged by glucose and oxygen deprivation. This reaffirmed the hypothesis of a specific, tissue protection-mediating receptor. Evidences for the implication of another heterodimeric receptor complex, consisting of an a-chain of the leukemia inhibitory factor receptor (LIFRa) and interleukin-6-transducer-chain gp130, a LIFR/gp130 complex, in EV3-mediated neuroprotective effects were provided (Bonnas 2009). This finding together with results from Brines and colleagues (Brines et al. 2004) suggest that different tissues or cell types may respond to Epo through different receptors.

The concept of distinct heterodimeric receptors expressed in different tissues to convey protective messages from Epo-like molecules in response to harmful stimuli is in agreement with the hypothesis of a pre-vertebrate evolution of a tissue protective Epo/EpoR-like signaling system. It was shown that rhEpo increased the viability of primary cultured locust brain neurons in the presence of apoptosis-inducing stimuli (Ostrowski et al. 2011). The presence of a receptor that can be activated by Epo in invertebrates was confirmed by subsequent pharmacological studies. Epo-mediated neuroprotection of locust brain neurons required activation of the JAK/STAT transduction pathway (Miljus et al. 2014), which is also involved in Epo-induced responses of mammalian neurons. The molecular identity of the receptor through which neuroprotective effects of rhEpo are transmitted in insect neurons is not known yet. In addition to its association with JAK, the insect receptor may share more characteristics with vertebrate EpoR, e.g. receptor-mediated endocytosis of the ligand/receptor 
complex after binding of Epo. Many cytokine receptors, including EpoR (Becker et al. 2010), are internalized constitutively even in the absence of their ligands. However, cytokine-induced endocytosis is usually faster and more pronounced than the constitutive endocytosis (Basquin \& Sauvonnet 2013; Becker et al. 2010; Hémar et al. 1994). Epo-stimulated endocytosis could be detected as the difference in endocytosis rate of rhEpo-stimulated primary cultures compared to the endocytosis rate of non-stimulated primary cultures. Alternatively, detection of labeled Epo molecules in endocytotic vesicles would be indicative for Epo-stimulated endocytosis.

In order to advance the characterisation of the yet unidentified neuroprotective Epo receptor, I have studied Epo-stimulated endocytosis in primary cultured locust brain neurons. Locust brain neurons were incubated with the fluorescent dye FM1-43 to quantify endocytotic activity. In addition, I have undertaken attempts to label rhEpo with a fluorescent probe using the $\mathrm{N}$ hydroxysuccinimide ester-mediated chemical reaction and detect its presence in endocytotic vesicles of locust brain neurons. To obtain further information about the functional structure of the insect Epo-binding receptor, I have exposed hypoxia-subjected locust brain neurons to the non-erythropoietic human splice variant EV3. Shared neuroprotective potency of EV3 in mammals and insects, in the absence of erythropoietic effects, would suggest a greater similarity of the unidentified nervous Epo receptors across phyla than between mammalian hematopoietic and neuroprotective receptors. 


\section{II.2 Material and Methods}

\section{II.2.1 Examining the effect of the human Epo splice variant EV3 on neuronal survival during hypoxia-induced apoptosis}

Primary cultures of locust brain cells were established from fourth-instar nymphs of Locusta mig ratoria (Hintze, Berlin, Germany) as described in chapter I (I.2.2). The pooled and dissociated cells from 12 locust brains were equally distributed to 6 culture dishes. Cell cultures were maintained for 5 days under normal growing conditions. Afterwards, culture medium was removed and replaced with serum-free medium (L15/0.5\%GM) with or without rhEpo $(4 \mathrm{U} / \mathrm{ml})$ or EV3. Final concentrations of applied EV3 in a first experiment were $0.33 \mathrm{ng} / \mathrm{ml}, 3.33 \mathrm{ng} / \mathrm{ml}, 33.30 \mathrm{ng} / \mathrm{ml}$ corresponding to $0.04,0.4$ and $4 \mathrm{U} / \mathrm{ml}$ rhEpo, whereas in the following series of experiments final concentrations of $2.08,4.17$ and $8.33 \mathrm{ng} / \mathrm{ml}$ corresponding to $0.25,0.5$ and $1 \mathrm{U} / \mathrm{ml}$ rhEpo were chosen. A stock solution of the non-erythropoietic human Epo variant EV3 (Iba Technologies, Göttingen, Germany) (Bonnas 2009) with the concentration of $0.194 \mathrm{mg} / \mathrm{ml}$ was maintained in PBS and diluted with culture media to achieve the desired final concentrations. After $12 \mathrm{~h}$ of incubation with rhEpo or EV3 or with L15 medium only (control cultures) under normoxic conditions, cell cultures were exposed to hypoxia $\left(\mathrm{O}_{2}\right.$ level $\left.\leq 2 \%\right)$ for $36 \mathrm{~h}$. Subsequently, cells were maintained for another $12 \mathrm{~h}$ in normal atmosphere (Fig. 13). Control cultures were kept under normoxic conditions for the whole period $(12 \mathrm{~h}+36 \mathrm{~h}$ +12 h). Finally, cells were fixed with $4 \%$ paraformaldehyde in $0.1 \mathrm{M}$ phosphate buffer for $30 \mathrm{~min}$ at RT, rinsed five times with PBS (pH 6.9) and incubated with DAPI (Sigma-Aldrich Chemie, Schnelldorf, Germany) dissolved in PBS at a final concentration of $100 \mu \mathrm{g} / \mathrm{ml}$ for $30 \mathrm{~min}$ in the dark. Excess dye was removed by several washes with PBS and cell-containing coverslips were mounted on microscopic slides in DABCO (Carl Roth, Karlsruhe, Germany). 


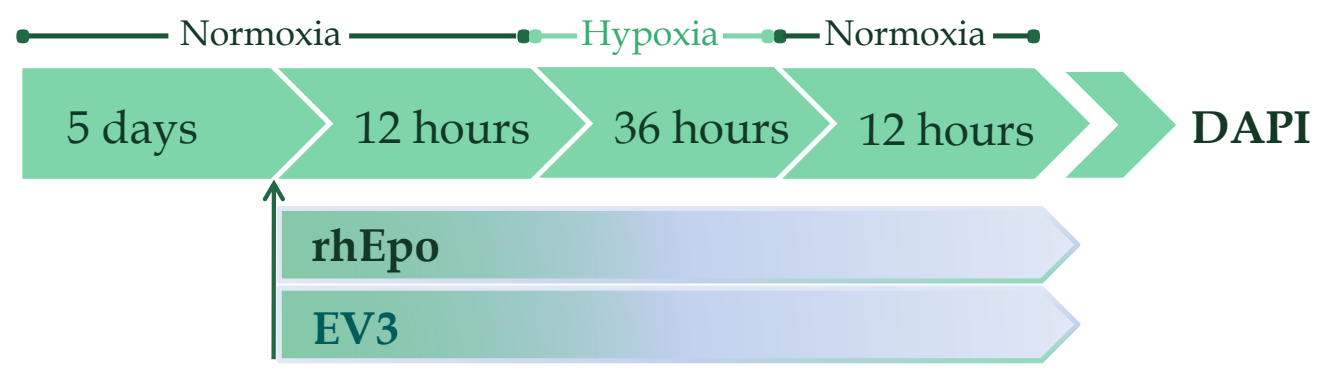

Figure 13. Schematic drawing of the experimental procedure to evaluate a potential neuroprotective effect of EV3 on primary cultured Locusta migratoria brain neurons.

Analysis of neuronal survival was performed using an epifluorescence microscope (Zeiss Axioskop; 40x objective) equipped with a Spot CCD camera (Invisitron, Puchheim, Germany). Two continuous rows of non-overlapping photographs (usually 60 per culture) to the right and the left of the center extending over the entire coverslip were taken from each culture. Nuclear morphology was used to distinguish viable from non-viable cells. Nuclei of living cells contain a discontinuous patchy DAPI labeling, reflecting intact chromatin structure, while nuclei of late apoptotic or dead cells are characterized by condensed continuous DAPI labeling. Total numbers of nuclei and numbers of intact nuclei were counted on each photograph and the portion of living cells determined for each culture.

Statistical analysis: In order to compare different experiments, the portions of living cells in different treatment groups of one experiment were normalized to the portion of living cells in the untreated control culture (set as 100\%), giving the relative portion of living cells in percentages displayed in typical box plot graphs. The software package OriginPro (version 8.5, OriginLab Corporation) was used to generate initial diagrams and to perform statistical analyses. The non-parametric Kruskal-Wallis test was used to identify differences in data population distributions and the Mann-Whitney $U$ test for unmatched samples was used to evaluate the differences between two groups. $P$ values were 
corrected with the Benjamini-Hochberg procedure (Benjamini \& Hochberg 1995; Groppe et al. 2011).

\section{II.2.2 FM1-43 dye loading protocol and rhEpo-induced endocytosis}

To study potential effects of rhEpo on endocytotic vesicle formation, cultured locust brain cells were exposed to FM1-43 dye (Biomol, Hamburg, Germany). The chemical structure of FM dyes makes them soluble in polar solvents, like water (hydrophilic, positively charged head group), but also ensures that the fluorescent signal of the fluorophore is emitted only in a hydrophobic environment (lipophilic tail) (Fig. 14). The lipophilic tail region facilitates the incorporation of the dye into phospholipids of the outer leaflet of the plasma membrane. The vesicles that are subsequently endocytosed will contain dye molecules inserted into their inner membrane leaflet. Polar head groups of the dye will prevent the dye from flipping over to the opposite leaflet of the membrane. This assures that, once trapped in endocytotic membrane compartments via endocytosis, a dye cannot escape the vesicles (the dye can only escape by exocytosis of the vesicles) (Gaffield \& Betz 2006).

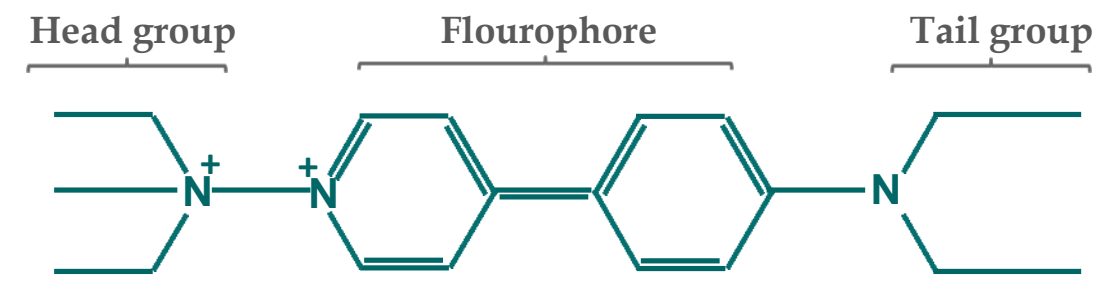

Figure 14. Structure of the FM1-43 dye molecule. The lipophilic tail region of the dye (right) causes its insertion into membrane phospholipid layers. Two aromatic rings connected with a double bond (middle) create the fluorophore. The positively charged head group (left) prevents the dye from flipping across the membrane. Adapted by permission from Macmillan Publishers Ltd: Nature Protocols (Gaffield \& Betz 2007), copyright (2007).

Primary cultures of locust brain neurons were prepared as described in chapter I (1.2.2). The pooled and dissociated cells from 8 locust brains were equally distributed to 4 culture dishes and grown for 5 days under normal culturing 
conditions. Before exposure to FM1-43 dye cell cultures were pre-incubated for 15 min with L15 medium containing $10 \mathrm{mM}$ of the calcium chelator ethylenediaminetetraacetic acid (EDTA, AppliChem, Darmstadt, Germany) at RT to suppress calcium dependent exocytosis (L15/10 mM EDTA). Subsequently cultures were treated with rhEpo $(8 \mathrm{U} / \mathrm{ml})$ dissolved in L15/10 mM EDTA containing diluted FM1-43 dye at a final concentration of $8 \mu \mathrm{M}$ (L15/10mM EDTA/FM1-43) for 2 and $10 \mathrm{~min}$ at RT, protected from light. Control cultures were incubated with L15/10mM EDTA/FM1-43 for the same period of time. From this step on throughout the whole further procedure cultures were kept in the dark, taking care to minimize their exposure to the light. After $2 \mathrm{~min} / 10 \mathrm{~min}$ treatment, cell cultures were rinsed shortly three times (each time for few seconds) with fresh L15/10 mM EDTA medium to wash away extracellular FM dye and rhEpo and fixed with 4\% paraformaldehyde dissolved in $0.1 \mathrm{M}$ phosphate buffer for $30 \mathrm{~min}$ at RT (Fig. 15). After two 5-minute washes with PBS ( $\mathrm{pH}$ 6.9) cultures were rinsed several times with PBS containing $0.1 \%$ Triton-X-100. This step improved visualization of endocytotic vesicles during imaging, reducing the amount of nonspecific staining coming from membrane remnants of dying cells within cultures (Gaffield \& Betz 2006). To stain nuclei cultures were incubated with DAPI dissolved in PBS at a final concentration of $100 \mu \mathrm{g} / \mathrm{ml}$ for $30 \mathrm{~min}$ at RT. Finally, excess dye was removed by several washes with PBS and cell-containing coverslips were mounted on microscopic slides in DABCO. Preparations were stored for at least two days at $4^{\circ} \mathrm{C}$ in the dark, which increased the intensity of FM1-43 fluorescence.

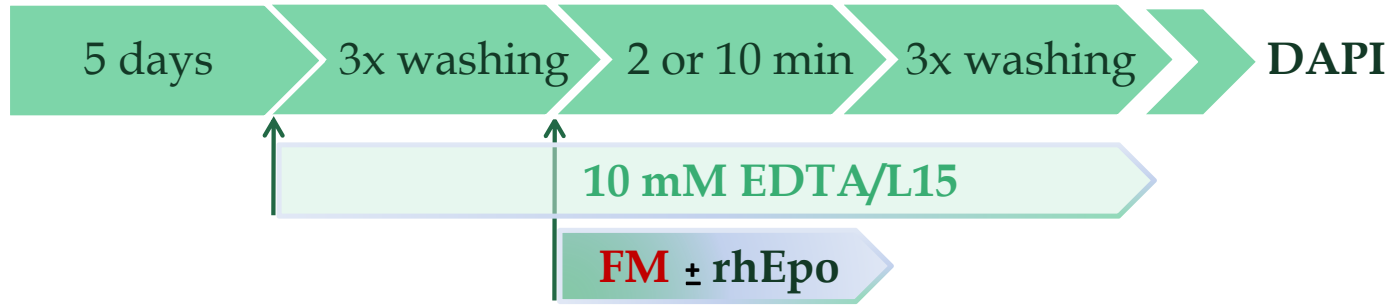

Figure 15. Schematic drawing of the experimental procedure to study rhEpo-stimulated endocytosis in primary cultures of Locusta migratoria brain neurons. 


\section{II.2.3 Analysis of endocytotic vesicles in primary cultured locust brain cells}

Analysis of FM1-43 dye-containing intracellular vesicles was performed using laser scanning confocal microscopy (Leica TCS SP2, Leica Microsystems, Wetzlar, Germany) with a $63 x$ oil immersion objective. Without prior examination of FM1-43 fluorescence (to prevent bias in the selection of evaluated neurons), DAPI-fluorescence was used to select regions of the coverslip that contained at least 10 neurons that were physiologically intact at the time of fixation. At least 10 selected regions (on average 126 individual neurons) were analyzed in each culture. For each preselected region of the culture FM1-43 fluorescence was scanned at step sizes of $1 \mu \mathrm{m}$ throughout the entire extension of the neurons along the z-axis (24-28 individual scans), at $1024 \times 1024$ pixel resolution. The 514-nm line of an argon laser was used to excite the FM1-43 dye and emitted fluorescent signals were collected from 530 to 700 nm. The number of FM1-43 labeled vesicles was counted for each neuron. Each optical section from the stacks along the z-axis through the neurons was analyzed to determine vesicle numbers. Exact determination of vesicle numbers in neurons of all sizes was possible up to 15 vesicles per cell. High densities of vesicles prevented exact counting due to overlap in the microscopic scans. Neurons were therefore categorized into classes with $\leq 5,6-10,11-15$ and $>15$ vesicles and the percentage of neurons in each class was calculated for each culture. Statistical comparison between control and rhEpo-treated neurons was performed with two sample T-tests.

To confirm the results of manual vesicle counting the same confocal scans were subjected to automatic quantification of labeled vesicles. The method was established by Dr. Bart Geurten (Department of Cellular Neurobiology, University of Göttingen). The first step of analysis included the detection of individual cells. The source image was converted into binary image format with an $8 \%$ contrast threshold. The binary image was then processed with a two dimensional Gaussian filter ( $\sigma 10$ pixels) and subjected to Hough 
transformation (for a review on Hough transformations see (Mukhopadhyay \& Chaudhuri 2015)). Imfindcircles algorithm of Matlab (Mathworks, USA) was used for detection of the cells as circles with radii of 3.5 to $23.3 \mu \mathrm{m}$ (equivalent to $15-100$ pixels). If more than $9.3 \mu^{2}$ (equivalent to 40 pixels $^{2}$ ) were black the cell was regarded as being damaged or dead and discarded from further evaluation. Furthermore, cells whose centers were located inside the radius of another cell were also excluded from analysis. The second step of analysis detected labeled vesicles. For this purpose an $80 \%$ threshold filter was applied to the source image resulting in a binary image that displayed labeled vesicles. Gaps between labeled pixels were closed by image morphology (4 pixels) (for a review on image morphology techniques see (Soille 2003)). Vesicles were detected as ellipses via Hough transform that was described by Basca and colleagues (Basca et al. 2005; Xie \& Ji 2002) and implemented in Matlab by Martin Simonovsky. The algorithm to detect ellipses was set to a major axis of 0.4 to $1.6 \mu \mathrm{m}$ (equivalent to 3 to 7 pixels). Overlying vesicles and fluorescent objects lying outside of detected cells were discarded. The third step of analysis involved combining optical sections of the detected cells to three dimensional objects and assignment of detected vesicles. Positions of centers of all cells in two frames were treated like the vertices of a bipartite graph in a minimum cost matching problem that was solved with the Hungarian algorithm (Kuhn 1955; Kuhn 1956). Labeled vesicles were associated with the volume of single cells, and assignments with distances larger than the radius of the respective cells were discarded. Finally, total volumes of vesicles per individual cell were calculated and plotted in box-whisker plots. For statistical analysis total volumes of vesicles per cell in control and Epo-treated cultures were compared by permutation tests (Fisher's exact test) on the differences between the medians of different experimental groups (Fisher 1954; Ernst 2004). 


\section{II.2.4 Anti-HRP immunocytochemistry and FM1-43 staining}

FM1-43 dye was applied to cell cultures according to the above described protocol. In brief, after preincubation in L-15/10 mM EDTA medium for 15 minutes cell cultures were treated with rhEpo $(8 \mathrm{U} / \mathrm{ml})$ dissolved in L-15/10 mM EDTA medium containing diluted FM1-43 dye at a final concentration of 8 $\mu \mathrm{M}$ for 2 and $10 \mathrm{~min}$ at RT, protected from light. After several short washes (3 times for few seconds) with fresh L-15/10 mM EDTA medium cell cultures were fixed with $4 \%$ paraformaldehyde dissolved in $0.1 \mathrm{M}$ phosphate buffer for $30 \mathrm{~min}$ at RT. Following three washes with 0.1 M Phosphate Buffer, three washes in PBS and three washes in PBS with 0.1\% Triton-X-100 (each for $5 \mathrm{~min}$ ), cells were incubated for $1 \mathrm{~h}$ with blocking solution containing $5 \%$ normal goat serum (Jackson Immuno Research Laboratories, West Grove, Pennsylvania, USA) and $0.25 \%$ bovine serum albumin (MP Biomedicals, Heidelberg, Germany) dissolved in PBS with $0.1 \%$ Triton-X-100. Primary antibody anti-HRP from rabbit (Sigma-Aldrich, dilution 1:500, v/v, in blocking buffer) was incubated over night at $4^{\circ} \mathrm{C}$. After several washes in PBS with $0.1 \%$ Triton-X-100 a secondary antibody Cy5-coupled goat anti rabbit (Jackson Immuno Research Laboratories, dilution 1:300 in PBS containing 0.1\% Triton-X-100) together with DAPI (1:1000) was applied for $1 \mathrm{~h}$ at RT. After washing away excess antibody and DAPI (5 washes in PBS with $0.1 \%$ Triton-X-100), coverslips with labeled cells were mounted on microscopic slides using a drop of DABCO.

Anti-HRP staining in conjunction with FM1-43 staining was analyzed by confocal fluorescence microscopy (Leica TCS SP8, Leica Microsystems, Wetzlar, Germany) using a 63x glycerol immersion objective. FM1-43 fluorescence was exited with the $514 \mathrm{~nm}$ line of an argon laser, while Cy5 fluorescence was excited with the $633 \mathrm{~nm}$ line of a HeNe laser. The emitted fluorescence was collected from 530 to $600 \mathrm{~nm}$ and 680 to $790 \mathrm{~nm}$ respectively. The nuclear dye (DAPI) was excited using the $405 \mathrm{~nm}$ line from a diode laser and the emission was detected from 420 to $480 \mathrm{~nm}$. Each stack was sequentially scanned (DAPI and Cy5 fluorescence in one sequence and FM 1-43 fluorescence in a second 
sequence) in z-direction with $1024 \times 1024$ pixel resolution in the xy-plane, $400 \mathrm{~Hz}$ scanning speed and $\sim 1 \mu \mathrm{m}$ distance between each two of 25 to 35 optical sections along the z-axis.

\section{II.2.5 Labeling of rhEpo}

N-hydroxysuccinimide (NHS) ester reaction chemistry (Fig. 16) was used to fluorescently label rhEpo. NHS esters are reactive groups formed by carbodiimide-activation of carboxylate molecules. NHS ester-activated groups react strongly with primary amino groups present in the side-chain of lysine amino acid residues and at the amino terminus of the protein. This reaction leads to the formation of a stable amide bond between the primary amino group and the flourophore group of the NHS ester-fluorophore complex, while NHS is released (Nanda \& Lorsch 2014).

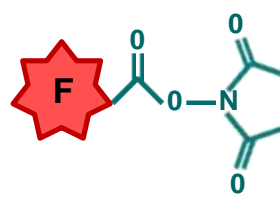

Activated NHS Ester

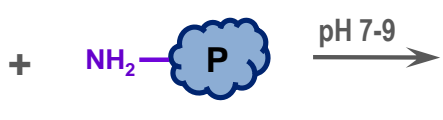

Protein with Primary Amine

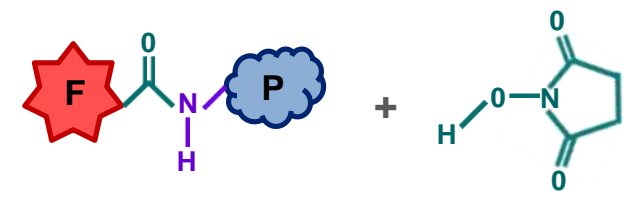

Stable Conjugate with amide bond

NHS

Figure 16. N-hyd roxysuccinimde (NHS) ester-mediated chemical reaction between the a minereactive group of the NHS ester-fluorophore complex and the primary amino group of a protein. F-fluorophore; P-protein. Adapted and used with permission from Thermo Fisher Scientific, copyrighted 2016.

A spin concentrator (Corning Spin-X UF $500 \mu$ l Concentrator, Sigma-Aldrich), an ultrafiltration device with a polyethersulfone membrane, was used to concentrate rhEpo (NeoRecormon, Roche, Welwyn Garden City, UK) to the desired concentration. The procedure of concentrating consisted of filling the concentrator with up to $500 \mu \mathrm{l}$ of rhEpo solution, centrifuging the solution in a centrifuge with a fixed angle rotor (Heraeus, Fresco 21 centrifuge, Thermo Fisher Scientific, Braunschweig, Germany) at a speed of $14000 \times \mathrm{g}$ for 5-10 min 
at $20^{\circ} \mathrm{C}$, and discarding the filtrate solution from the filtrate container. By repeating this procedure several times the starting volume of rhEpo was reduced by approximately 50 times. Given that the solvent of rhEpo contained glycine, a primary amine that competes with primary amino groups of proteins for the NHS ester-mediated chemical reaction, it needed to be replaced with a more suitable buffer. To achieve this, the spin concentrator that contained the concentrated rhEpo was filled with PBS ( $\mathrm{pH} 7.4)$, centrifuged as described above and the filtrate was discarded. The process was repeated 10 times with 5 min pauses between cycles during which the rhEpo sample was kept on ice. Finally, the sample with rhEpo was recovered from the bottom of the concentrate pocket and its concentration determined spectrophotometrically by measuring the absorbance of the sample at $280 \mathrm{~nm}$ using a NanoDrop analyzer (ND-1000-v 3.7.1, Thermo Fisher Scientific).

To fluorescently label rhEpo a Monolith NTTM protein labeling kit red-NHS (NanoTemper Technologies, Munich, Germany) was used. Following manufacturer's instructions, the solvent of rhEpo was exchanged with labeling buffer using a spin column with resin bed (both provided with the kit). RhEpo was diluted with labeling buffer to approximately $10 \mu \mathrm{M}$ and a volume of 100 $\mu 1$. Fluorescent dye (NT-647) was dissolved in dymethylformamide (DMF, Sigma-Aldrich) and diluted with labeling buffer to $240 \mu \mathrm{M}$ concentration and a volume of $100 \mu \mathrm{l}$. Solutions of rhEpo and dye were mixed in 1:1 volume ratio (200 $\mu$ l final volume) and incubated for $60 \mathrm{~min}$ at RT in the dark on a slowly rotating platform (Shaker DOS-10L, Neo Lab, Heidelberg, Germany), followed by overnight incubation at $4^{\circ} \mathrm{C}$ in the dark. Using a purification column provided with the kit, remaining free dye was retained in a column and thereby removed from the sample, while labeled rhEpo was collected from a column using PBS (pH 6.9) as an elution buffer.

To ensure complete elimination of the free dye, the sample was transferred to a spin concentrator and subjected to a buffer exchange process with PBS ( $\mathrm{pH}$ 6.9) applying the above described procedure. The process for buffer exchange was 
repeated 10 times with at least 5 min pauses between centrifugation cycles during which the sample with the labeled rhEpo was kept on the ice. After the last centrifugation cycle, the sample volume was adjusted to $100 \mu \mathrm{l}$ using PBS (pH 6.9).

\section{II.2.6 Assessment of the labeling efficiency and purity of the rhEpo- fluorophore conjugate}

The concentration of labeled protein product and labeling efficiency were subsequently determined spectrophotometrically using a NanoDrop analyzer. To confirm that the labeled product was indeed rhEpo and that the sample was completely free of unbound dye, sodium dodecyl sulphate polyacrylamide gel electrophoresis (SDS-PAGE) was performed and gels fluorescently scanned. Furthermore, the same gels were blotted and analyzed with western blotting technique.

For SDS-PAGE, samples were mixed with equal volumes of protein sample buffer $(2 \mathrm{x}, 1 \mathrm{M}$ tris- $\mathrm{HCl}, \mathrm{pH}$ 6.8, 4\% SDS, 20\% glycerol, $0.02 \%$ bromophenol blue) containing $5 \% \beta$-merkaptoethanol (AppliChem), heated for $3 \mathrm{~min}$ at $95^{\circ} \mathrm{C}$ (Eppendorf Thermomixer Compact, Sigma-Aldrich) and loaded onto 12\% polyacrylamide gels. The gels were prepared by layering a stacking gel (tris buffer, pH 6.8; 5\% polyacrylamide) (30\% acrylamide/bis-acrylamide, SigmaAldrich) on top of a separating gel (tris buffer, $\mathrm{pH} 8.8 ; 12 \%$ polyacrylamide). The PageRuler Plus prestained protein ladder (Thermo Fisher Scientific) was used as a molecular weight marker. The electrophoresis was run at $120 \mathrm{~V}$ in Laemmli buffer (1x, 25mM tris, $190 \mathrm{mM}$ glycine, 0.1\% SDS) for approximately 100 minutes (PowerPac ${ }^{\mathrm{TM}}$ Basic Power Supply, Bio-Rad Laboratories, Munich, Germany) and stopped when samples reached the end of the gel. The gels were subsequently scanned using a Typhoon Scanner FLA 9500 (GE Healthcare Life Sciences, Freiburg, Germany) with a 635-nm laser excitation and the LPR-Ch2 filter (665 $\mathrm{nm}$ long pass). 
After fluorescent scanning, a semi-dry blotting system was used for the electrophoretic transfer of proteins from the polyacrylamide gels to nitrocellulose membranes (Roti NC, Carl Roth). Before assemblage into a "sandwich", gels, membranes and Whatman sheets (Protean XL, Bio-Rad Laboratories) were soaked in transfer buffer (25mM tris, $192 \mathrm{mM}$ glycine, 20\% methanol) for at least $15 \mathrm{~min}$. The transfer was performed for $70 \mathrm{~min}$ at $15 \mathrm{~V}$ (Transblot SD transfer cell, Bio-Rad Laboratories). After one hour blocking of the membranes in blocking buffer containing $1 \%$ bovine serum albumin dissolved in tris buffered saline (TBS, $20 \mathrm{mM}$ tris, $137 \mathrm{mM} \mathrm{NaCl}, \mathrm{pH}$ 7.5) with $0.05 \%$ Tween (TBS-T) at RT on a shaker, the membranes were incubated with anti-Epo antibody from goat (1:1000, v/v, N-19, Santa Cruz Biotechnology, Heidelberg, Germany) dissolved in TBS-T overnight at $4^{\circ} \mathrm{C}$. Following 5 washes with TBS-T for $5 \mathrm{~min}$ at RT on a shaker, membranes were incubated with the secondary peroxidase-conjugated antibody (rabbit anti-goat, 1:1000, v/v, Jackson Immuno Research Laboratories) for 1 hour at RT on a shaker. Membranes were afterwards rinsed 5 times with TBS-T for $5 \mathrm{~min}$ at RT on a shaker and developed using a Peroxidase/Luminol-Enhancer reagent (Super Signal West Pico Chemiluminescence Substrate, Thermo Fisher Scientific). Solution A (Peroxide) and solution B (Luminol/Enhancer) were mixed in 1:1 ratio and applied to the membranes, followed by $5 \mathrm{~min}$ incubation at RT in the dark, as instructed by the manufacturer. Immunoreactive protein bands were visualized using the enhanced chemiluminescence detection system (ECL, Fluor Chem FC2, Cell Biosciences, Protein Simple, San Jose, California, USA), according to manufacturer's instructions.

\section{II.2.7 Ligand-receptor binding and internalization assay in primary cultures of locust brain neurons using fluorescently labeled rhEpo}

Primary cultures of locust brain neurons were prepared and maintained as described in chapter I (1.2.2). After 5 days in vitro, the culture medium was replaced with serum-free medium (L15/0.5\%GM) and cell cultures left for $2 \mathrm{~h}$ 
at $27^{\circ} \mathrm{C}$ in a humidified normal atmosphere (Incubator, Heraeus Instruments). A ligand-receptor binding assay was performed by incubating locust neuronal cultures with L15/0.5\% GM containing $0.023 \mu \mathrm{M}$ fluorescently labeled rhEpo for $10 \mathrm{~min}$ at $27^{\circ} \mathrm{C}$ in an incubator. Cells were washed by transferring the cellcontaining coverslips to a new culture dish filled with fresh L15/0.5\% GM. Cellcontaining coverslips were transferred in this way 3 times (each time to a new culture dish filled with fresh L15/0.5\% GM) and finally fixed with $4 \%$ paraformaldehyde in $0.1 \mathrm{M}$ phosphate buffer for $40 \mathrm{~min}$ on ice protected from light. Control cultures were treated in the same way but instead of fluorescently labeled rhEpo, non-labeled rhEpo was incubated. After several washes with 0.1 M phosphate buffer and 2 washes with PBS, each for 5 min at RT in the dark, the cells were incubated with DAPI dissolved in PBS at a final concentration of $100 \mu \mathrm{g} / \mathrm{ml}$ for $20 \mathrm{~min}$ in the dark, to counterstain nuclei. Following several washes with PBS, cell-containing coverslips were mounted on microscopic slides in DABCO.

Analysis of fluorescence was performed by confocal microscopy (Leica TCSSP8) with a $63 x$ glycerol immersion objective. As the fluorophore conjugated to rhEpo has an absorption maximum of $650 \mathrm{~nm}$ and an emission maximum at 670 $\mathrm{nm}$, the $633 \mathrm{~nm}$ line of HeNe laser was used to excite the fluorophore of the labeled rhEpo and emitted fluorescence signals were detected from 650 to 790 nm. To ensure comparability of cultures treated with fluorescently labeled rhEpo and untreated cultures, identical settings for laser intensity, gain and offset were used (for every preparation). Each region was scanned at steps of 1 $\mu \mathrm{m}$ throughout the entire extension of the neurons along the $\mathrm{z}$-axis, at $1024 \times 1024$ pixel resolution. The same regions were afterwards scanned through a differential interference contrast (DIC) prism to obtain bright-field images with increased contrast. Images of individual optical sections from the stacks were processed and overlaid using ImageJ software (National Institute of Health, Bethesda, USA). 


\section{II.3 Results}

\section{II.3.1 The non-erythropoietic Epo splice variant EV3 protects locust neurons from hypoxia-induced cell death}

The ability of the non-erythropoietic human Epo splice variant EV3 to protect locust brain neurons was studied after 5 days in vitro. Cell cultures derived from identical sets of locust brains were pretreated with rhEpo or EV3 for $12 \mathrm{~h}$ before exposure to hypoxic conditions. In a preliminary experiment a wider concentration range of EV3 was applied $(0.33 \mathrm{ng} / \mathrm{ml}, 3.33 \mathrm{ng} / \mathrm{ml}, 33.30 \mathrm{ng} / \mathrm{ml})$ to test for potential protective effects and determine the dose leading to a maximal effect.
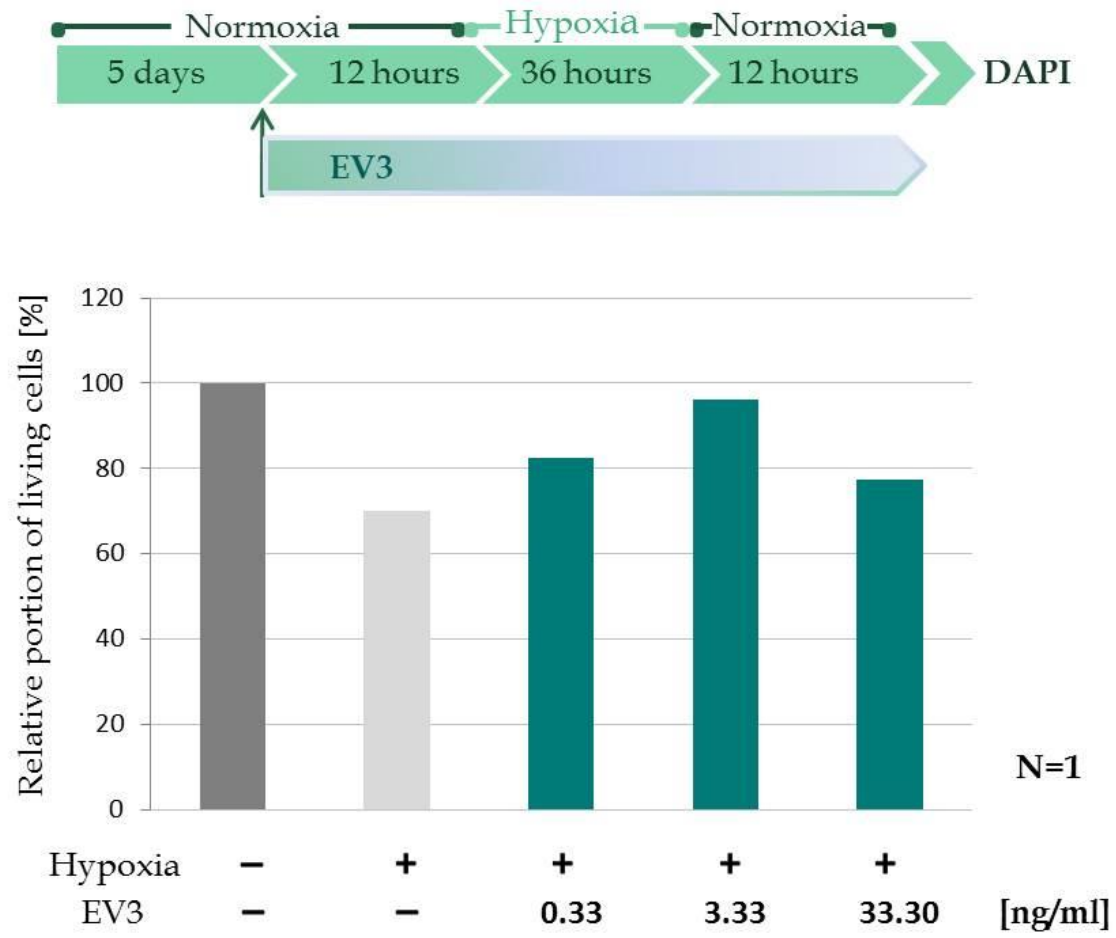

Figure 17. Dose-dependent effect of the human Epo splice variant EV3 on the viability of primary cultured locust neurons exposed to hypoxia. Initial experiment to determine a suitable range of EV3 concentraions for subsequent quantitative analysis (see Fig. 18).

Incubation of primary cultures for $36 \mathrm{~h}$ in a hypoxic environment $\left(\mathrm{O}_{2} \leq 2 \%\right)$ initiated apoptotic cell death and decreased the survival of cultured neurons to 
$70 \%$ compared to the control culture that was grown for the same duration under normoxic conditions (Fig. 17). Treatment of the locust primary neuronal cultures with EV3 for $12 \mathrm{~h}$ before onset of hypoxia reduced apoptotic cell death and supported cell survival. The protective effect of EV3 was dose-dependent, with best neuroprotection achieved with $3.33 \mathrm{ng} / \mathrm{ml}$ EV3, while neuroprotective effects of both, lower $(0.33 \mathrm{ng} / \mathrm{ml})$ and higher $(33.30 \mathrm{ng} / \mathrm{ml})$ EV3 concentrations were weaker.
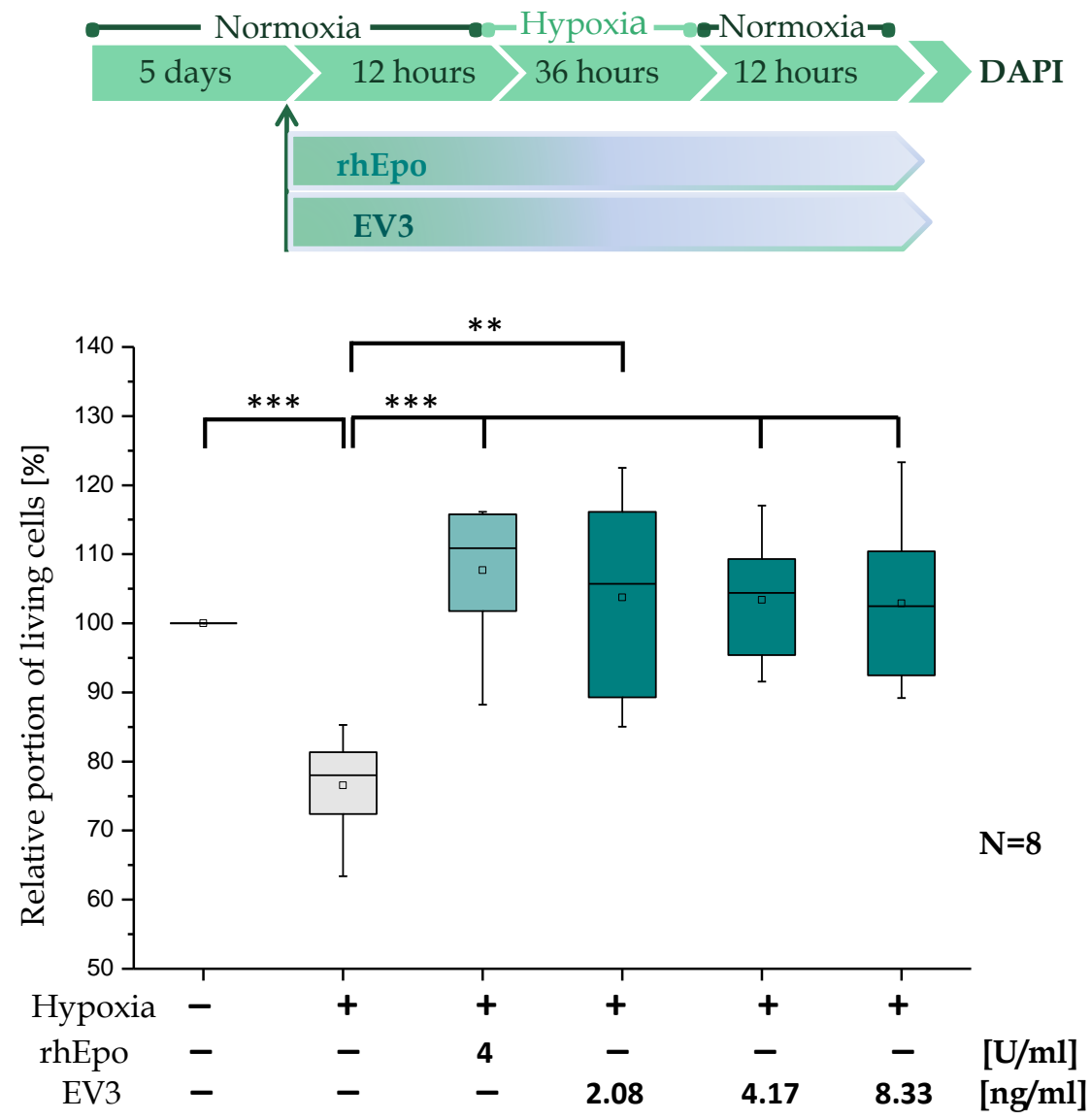

Figure 18. The human Epo variant EV3 protects primary cultured locust neurons from hypoxiainduced cell death. The neuroprotective effect of EV3 in three different concentrations (2.08, $4.17,8.33 \mathrm{ng} / \mathrm{ml}$ corresponding in weight to $0.25,0.5,1.0 \mathrm{U} / \mathrm{ml} \mathrm{rhEpo}$ respectively) is comparable to the neuroprotective effect of $4 \mathrm{U} / \mathrm{ml}$ rhEpo. Statistics: Kruskal-Wallis ANOVA with Mann-Whitney $U$-test for unmatched samples and Benjamini-Hochberg-correction of $P$ values. ${ }^{* *} P<0.01,{ }^{* * *} P<0.001$. 
After $12 \mathrm{~h}$ of incubation in growth medium only (two control cultures), in a medium supplemented with $4 \mathrm{U} / \mathrm{ml}$ rhEpo, and medium containing three different concentrations of EV3 $(2.08,4.17$ and $8.33 \mathrm{ng} / \mathrm{ml})$, locust neuronal cultures (except one control culture that was kept under normoxia during the whole experimental procedure) were subjected to hypoxia $\left(\mathrm{O}_{2}\right.$ level $\left.\leq 2 \%\right)$ for 36 h. At the end of the hypoxic period, cells were allowed to reoxygenate in normoxia for $12 \mathrm{~h}$ after which they were fixed and processed for analysis. The effects of rhEpo and EV3 on neuronal survival in eight individual experiments are summarized in the Figure 18. Continuous hypoxia for $36 \mathrm{~h}$ is a strong stressor that induced apoptosis and reduced the number of intact neurons. Compared to control cultures maintained under normoxic conditions, whose viability was normalized to $100 \%$, only $78.03 \%$ of neurons survived hypoxic treatment. Incubation of cell cultures with rhEpo $(4 \mathrm{U} / \mathrm{ml})$ completely prevented cell death, increasing the portion of surviving neurons (median: $110.90 \%)$. A similar neuroprotective effect was observed with EV3 treatment in all three different concentrations, namely $2.08 \mathrm{ng} / \mathrm{ml}, 4.17 \mathrm{ng} / \mathrm{ml}$ and 8,33 $\mathrm{ng} / \mathrm{ml}$ (corresponding to $0.25,0.5$ and $1.0 \mathrm{U} / \mathrm{ml}$ of Epo), which increased the portion of surviving neurons (medians: 105.74\%, 104.42\% and 102.49\%, respectively). Like rhEpo, EV3 completely prevented hypoxia-induced cell death of locust neurons. Full protection was accomplished even with the lowest EV3 concentration tested, which corresponds to approximately $6.25 \%$ of the most beneficial concentration of rhEpo in cultured locust brain neurons.

\section{II.3.2 Epo stimulates endocytosis in locust neurons}

To study the effect of rhEpo on endocytotic vesicle formation, primary cultured locust brain neurons were exposed to FM1-43 fluorescent dye for 2 min and 10 min. FM dyes bind to plasma membrane lipids and are internalized within vesicles that are formed during endocytosis. The neurons containing vesicles with incorporated FM1-43 dye were analyzed with confocal microscopy. Individual neurons contained between none and approximately 50 FM1-43 
labeled vesicles that were typically distinct from vesicles carrying strong antiHRP immunolabeling (Fig. 19). Anti-HRP labels a neuron-specific membrane protein (identified as an ATPase coded by the nervana gene in Drosophila) that is transported from the endoplasmic reticulum to the plasma membrane in strongly labeled vesicles. The absence of FM1-43 and anti-HRP co-labeled vesicles indicates distinct labeling of export- and endocytosed vesicles by the two markers.

$\mathbf{A}$
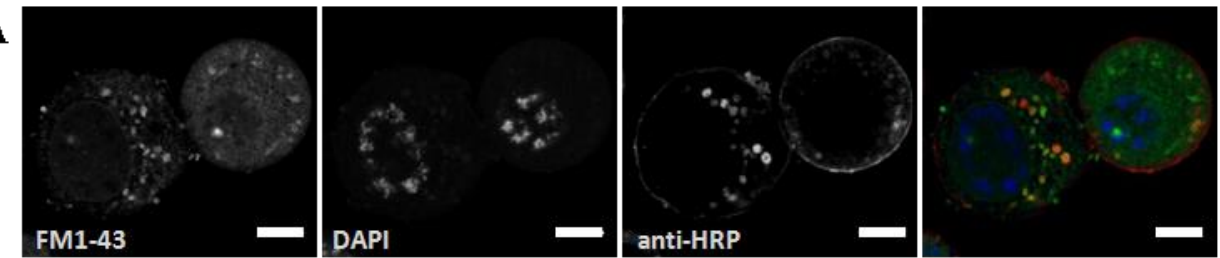

B
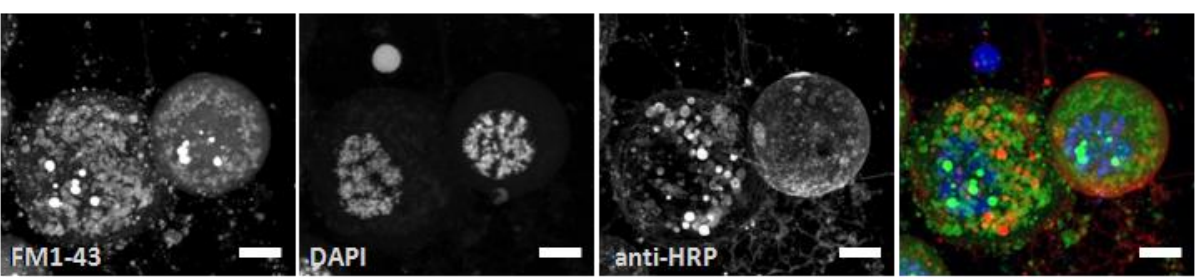

Figure 19. Physiologically intact locust brain neurons in vitro with endocy tosed FM1-43 labeled vesicles (green in the summary on the right), granular nuclear DAPI staining (blue) and antiHRP positive "export" vesicles (red) after 10 minutes exposure to $8 \mathrm{U} / \mathrm{ml} \mathrm{rhEpo.} \mathrm{A} \mathrm{Single}$ optical section. B Maximal projection of a series of optical sections through the same two neurons shown in A. Scale bars: $10 \mu \mathrm{m}$.

To study a potential stimulatory effect of rhEpo on endocytosis, two different approaches were used for analysis of the same confocal scans, an observerbased and an unsupervised software-based approach. In the first approach, physiologically intact neurons were identified based on nuclear DAPI staining and selected for analysis, prior to examination of FM1-43 fluorescence. The number of FM1-43 labeled vesicles was counted for each neuron. Neurons were categorized into four classes depending on the number of FM1-43 labeled vesicles and the percentage of neurons in each class was calculated. Data derived from 6 experiments with $2 \mathrm{~min}$ and 6 experiments with $10 \mathrm{~min}$ exposure to FM1-43 and a total number of 3029 evaluated neurons. The results are presented in Figure 20. 

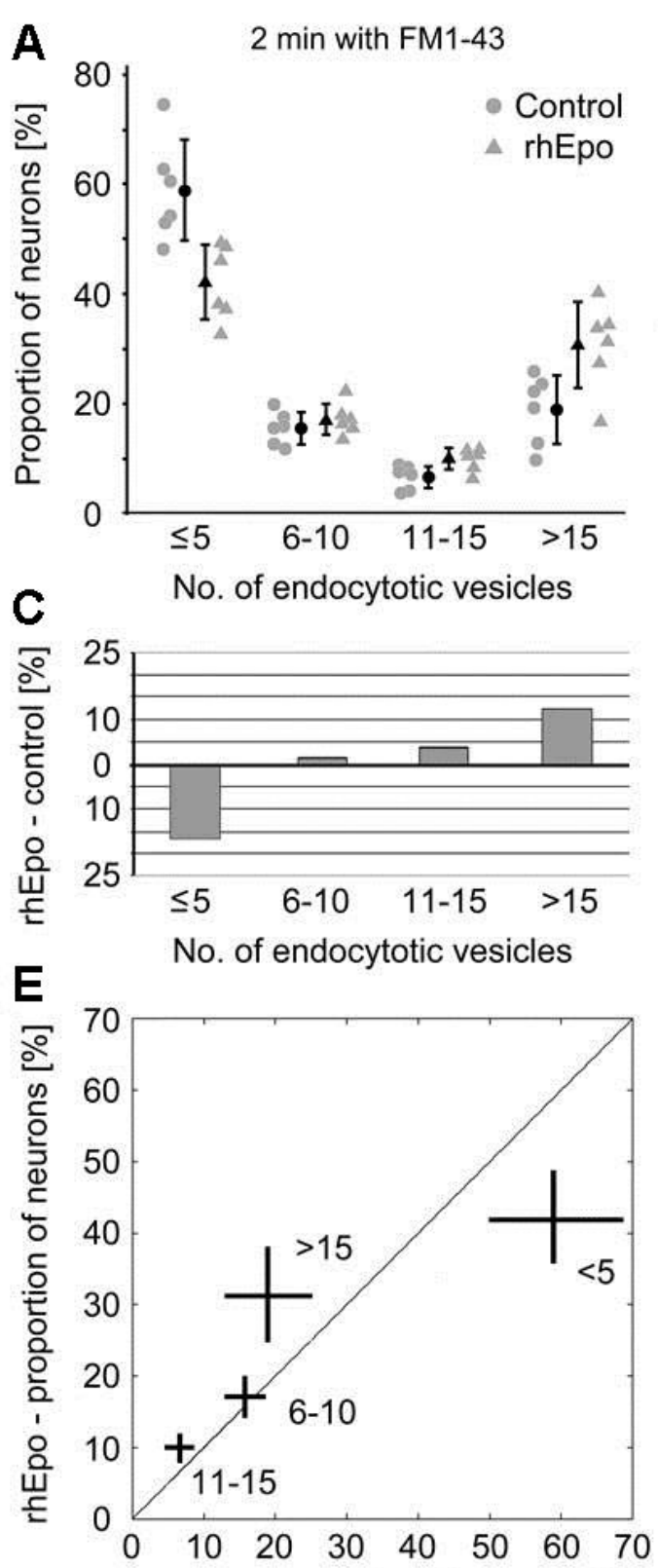

Control - proportion of neurons [\%]

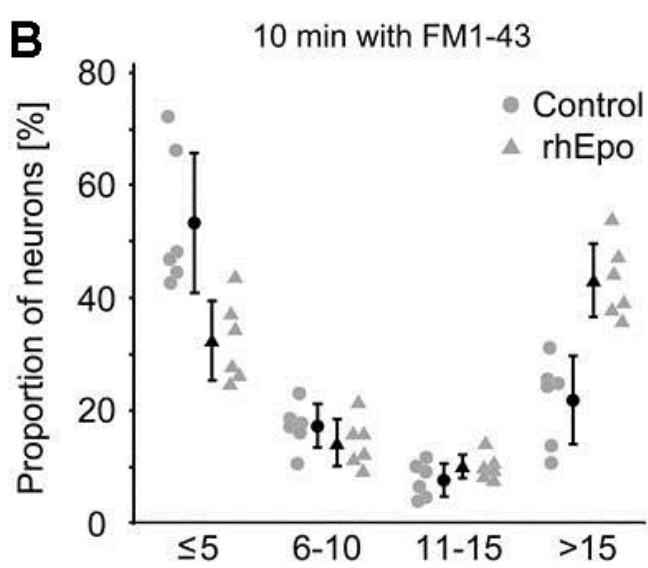

No. of endocytotic vesicles

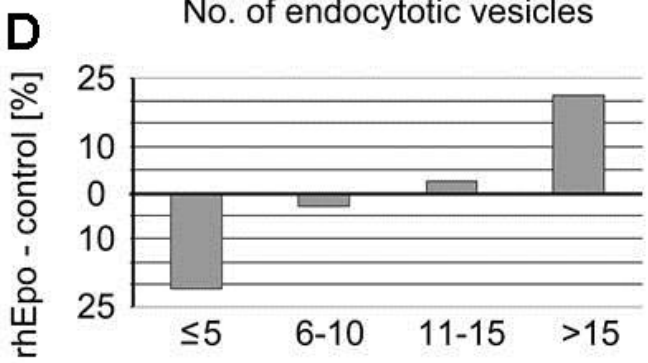

No. of endocytotic vesicles

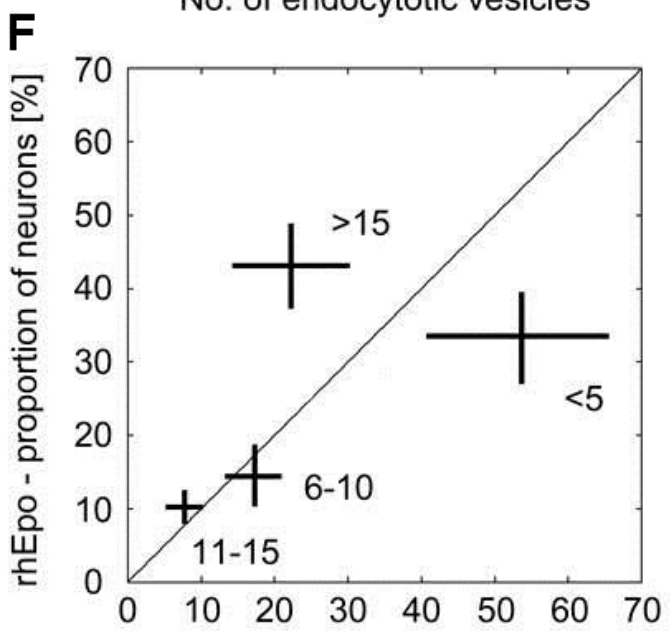

Control - proportion of neurons [\%]

Figure 20: RhEpo stimulates endocytosis in cultured locust brain neurons. A, B Proportion of neurons with $\leq 5,6-10,11-15$ and $>15$ labeled endocytotic vesicles after exposure to FM1-43 with or without rhEpo for $2 \mathrm{~min}(\mathrm{~A})$ and $10 \mathrm{~min}$ (B). C, D Differences of average proportions of neurons within each class between cultures treated with rhEpo and untreated cultures (rhEpotreated minus control). With both incubation times Epo leads to a reduction of neurons with lower numbers and an increase of neurons with higher numbers of endocytotic vesicles. $\mathbf{E}, \mathbf{F}$ Control (X-axis) versus rhEpo-treated ( $\mathrm{Y}$-axis) average proportions of neurons with $\leq 5,6-10,11$ 15 and > 15 endocytotic vesicles after exposure to FM 1-43 for $2 \mathrm{~min}$ (E) and $10 \mathrm{~min}$ (F). Standard deviations that do not overlap with the angle bisector indicate significant differences between control and rhEpo-treated neuronal cultures. Data derive from each 6 experiments with 2 and 10 min exposure to FM1-43 and a total of 3029 evaluated neurons. 
Untreated neuronal cultures displayed a basal rate of endocytosis. After $10 \mathrm{~min}$ incubation with FM1-43 solution, the proportion of cells within 4 different classes changed insignificantly compared to 2 min exposure to FM1-43 (Fig. 20A, B, round symbols). There was a slight tendency towards a decrease of the average proportion of neurons with $<5$ vesicles in the untreated cultures exposed to FM1-43 for $10 \mathrm{~min}(53.3 \%$ vs. 58.8\%) compared to the untreated cultures exposed to FM1-43 for 2 min. Untreated cultures exposed to FM1-43 for $10 \mathrm{~min}$ showed also a slight increase of the average proportion of neurons with $>15$ vesicles compared to the untreated cultures exposed to FM1-43 for $2 \mathrm{~min}$ $(21.8 \%$ vs. $18.9 \%)$ and even less pronounced increase of the average proportion of neurons with 6-10 vesicles and with 11-15 vesicles compared to the cultures exposed to FM1-43 for 2 min (17.3\% vs. 15.7\% and 7.7\% vs. 6.6\%). Statistical comparison of average proportions of neurons of each category between control cultures exposed to FM1-43 for $10 \mathrm{~min}$ and control cultures exposed to FM1-43 for 2 min showed that the observed differences were not significant. The data indicate that a portion of cultured neurons performs endocytosis independent from rhEpo stimulation.

Simultaneous incubation of cultures with rhEpo and FM1-43 for 2 min decreased the average proportion of neurons with $<5$ vesicles $(P=0.0057)$ compared to the untreated cultures exposed to FM1-43 for 2 min, while the average proportion of neurons with $11-15$ vesicles $(P=0.0200)$ and the average proportion of neurons with $>15$ vesicles $(P=0.0178)$ (Fig. 20A, E) was significantly increased. A similar shift towards neurons containing higher numbers of vesicles was observed after 10 min co-exposure of cultures to FM143 and rhEpo (Fig. 20B). Here, rhEpo decreased the average proportion of neurons with $<5$ vesicles $(P=0.0057)$ compared to untreated cultures, while the increase of the average proportion of neurons with $>15$ vesicles stimulated by rhEpo was highly significant compared to untreated cultures $(P=0.0005)$ (Fig. 20F). 
Therefore, during both 2 min and 10 min exposure to FM1-43, rhEpo decreased the average proportion of neurons with $<5$ vesicles and increased the average proportion of neurons with $>15$ vesicles. Differences in average proportions of neurons within each category between cultures treated with rhEpo and untreated cultures are displayed in Figures 20C and 20D. Significance levels of the observed differences are presented in Figures 20E and 20F in which the average proportions of neurons in each category are plotted with their standard deviations, using the $\mathrm{x}$-axis for untreated cultures and $\mathrm{y}$-axis for rhEpo-treated cultures. Standard deviations that do not cross the angle bisector indicate significant differences, confirming the calculated statistical differences mentioned above.

The same stacks of confocal microscopic scans were also subjected to a software-based detection of neurons and vesicles, followed by automatic quantification of the total vesicle volume per cell in the four experimental situations. In contrast to the observer-based analysis described above, in which physiologically intact neurons were identified based on nuclear DAPI staining, here, neurons were identified on the basis of round shape with algorithmassisted exclusion of irregularly-shaped cells from the analysis. The quality of cell- and FM1-43-labeled-vesicle detection is shown for 4 (out of 15) optical sections of one cultured neuron (Fig. 21A). The reconstruction of the cells detected in multiple optical sections of a stack to three dimensional objects and their association with labeled vesicles is shown exemplary for one stack in Figure 21B. The total volumes of vesicles per individual neuron obtained from automatic quantification are displayed in box-whisker plots (Fig. 21C). Data derived from 6 experiments and a total number of 1573 evaluated neurons. Comparison of averages of total endocytotic vesicle volume per neuron between untreated cultures exposed to FM1-43 for 2 and 10 min showed a timedependent accumulation of endocytosed vesicles $\left(10.23 \mu \mathrm{m}^{3}\right.$ vs. $10.93 \mu \mathrm{m}^{3}$, different with $P=0.0491)$. Co-incubation of rhEpo with the fluorescent dye FM143 significantly increased the total endocytotic vesicle volume after both 2 min 
$(P=0.0001)$ and $10 \mathrm{~min}(P=0.0335)$ compared to control cultures exposed to FM143 only (Fig. 21C).
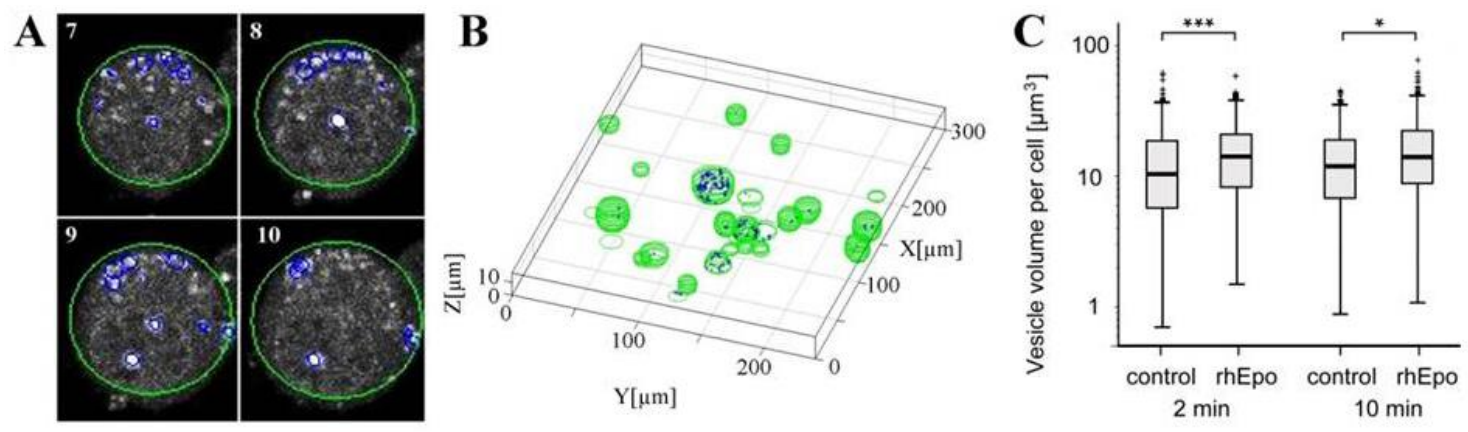

Figure 21: Automatic detection and quantification of FM1-43 labeled endocytotic vesicles. A Optical sections through one cultured locust brain neuron with automatically defined cellular borders (green) and labeled vesicles (blue). B Reconstructed neurons with associated vesicles from optical sections of one stack. C rhEpo increases the total volu me of FM1-43 labeled vesicles per neuron after 2 and $10 \mathrm{~min}$ incubation. Data derived from 6 experiments and a total of 1573 evaluated neurons. Fisher's exact test, ${ }^{* *} P<0.001,{ }^{*} P<0.05$.

\section{II.3.3 Determining the concentration of the rhEpo-fluorophore conjugate and the degree of labeling}

The concentration of rhEpo after labeling, purification and concentrating was calculated using the Beer-Lambert Law, which can be written as: $\mathbf{A}=\boldsymbol{\varepsilon}^{*} \mathbf{d} * \mathbf{c}$, where $\mathbf{A}$ is the measured absorbance, $\boldsymbol{\varepsilon}$ is the wavelength-dependent molar absorptivity coefficient or extinction coefficient, $\mathbf{d}$ is the path length and $\mathbf{c}$ is the unknown concentration of the absorbing species (Gilbert-Kawai \& Wittenberg 2014). Since proteins absorb at $280 \mathrm{~nm}$ and the absorption maximum of the fluorophore is $650 \mathrm{~nm}$, both values needed to be included in the equation in order to determine the concentration of labeled rhEpo. The absorbance of the rhEpo-fluorophore conjugate solution was measured at $280 \mathrm{~nm}\left(\mathrm{~A}_{280}\right)$ and at 650 nm (A650) using a NanoDrop spectrophotometer (module „Proteins and Labels“). To eliminate the contribution of the fluorophore absorption at $280 \mathrm{~nm}$, the correction factor (CF) was included into the equation. The extinction coefficient of the protein $\left(\varepsilon_{280}\right)$, an intrinsic property of each protein, is the 
absorbance of the protein at $280 \mathrm{~nm}$ in a $1 \mathrm{~cm}$ path length at a concentration of 1 $\mathrm{mg} / \mathrm{ml}$. Information about the extinction coefficient of rhEpo was obtained from the ExPASy Proteomic Server (ExPASy, 2011). Thus, the molar concentration of the rhEpo-conjugate was calculated as follows:

$\mathrm{C}[$ rhEpo-fluorophore $]=\mathrm{A}_{280}-\mathrm{A}_{650} \times \mathrm{CF} / \varepsilon_{280}=6.1 \times 10^{-6}[\mathrm{M}]$

The labeling efficiency or degree of labeling (DOL) represents an estimated number of fluorophore molecules conjugated to each protein molecule. In order to determine DOL, in addition to already known and previously determined numbers, the molar extinction coefficient of the fluorophore $(\varepsilon 650)$ had to be included into the equation. The DOL of the rhEpo-fluorophore conjugate was subsequently calculated using the following equation:

$\mathrm{DOL}=\mathrm{A} 650 / \varepsilon 650 \times \mathrm{C}[$ rhEpo-fluorophore $[\mathrm{M}]=1.4$

Hence, the determined degree of labeling suggested that on average rhEpo molecules were labeled with one or two molecules of fluorophore.

\section{II.3.4 Fluorescent scanning gel analysis and western blot analysis of the conjugate}

The purity of the labeled rhEpo was checked by fluorescence scanning of a separation gel and subsequent western blotting. RhEpo-fluorophore conjugate, non-labeled rhEpo and free fluorophore were subjected to one-dimensional SDS-PAGE. After separation of the protein markers, electrophoresis was stopped and the gel scanned at $635 \mathrm{~nm}$ using a Typhoon TM Scanner. A single fluorescent band at the molecular weight expected for rhEpo ( $38 \mathrm{kDa})$ was detected in the lane in which sample containing rhEpo-fluorophore conjugate was resolved (Fig. 22). This indicates that rhEpo has been labeled and that there were no free flourophores in the sample. As expected, an additional fluorescent band of a low molecular weight was detected in the lane in which free fluorophore was resolved via SDS-PAGE. 

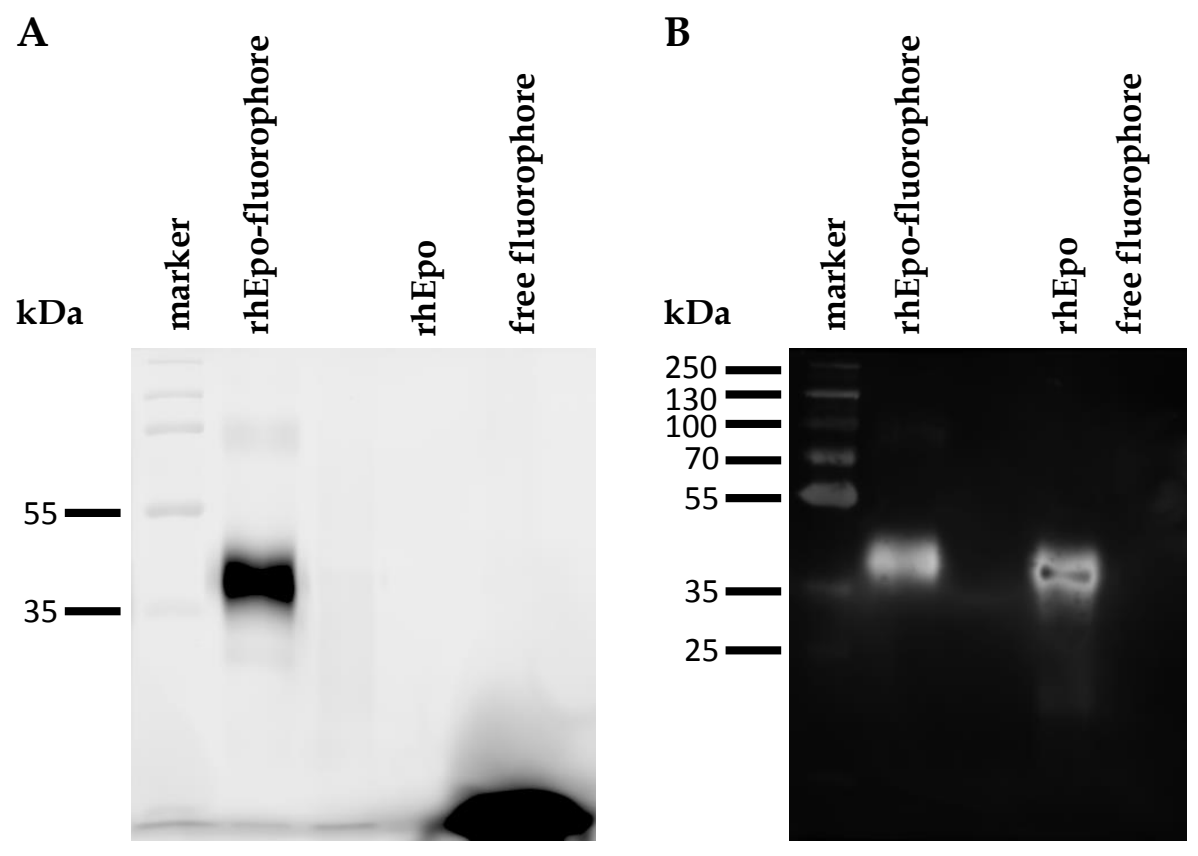

Figure 22. Fluorescent scanning gel analysis (A) and western blot analysis (B) of the rhEpofluorophore conjugate. The type of sample is indicated at the top of each lane. Nu mbers indicate the size in $\mathrm{kDa}$ and position of prestained molecular weight markers. Non-labeled rhEpo was resolved in the separate lane as a negative control for fluorescent scanning detection and positive control for immunochemical detection. Free fluorophore solution was resolved as a positive control for conjugate detection. A single fluorescent band in the sample containing the rhEpo-fluorophore conjugate indicates that rhEpo has been labeled and that there were no free fluorphores in the sample (A). RhEpo was detected by the Western blotting technique using anti-Epo antibody (B). The molecular weight of the labeled rhEpo (rhEpo-fluorophore conjugate) is similar to the molecular weight of non-labeled rhEpo.

To confirm that the fluorescent band with the molecular weight of $38 \mathrm{kDa}$ was indeed rhEpo, the proteins were subsequently transferred to a nitrocellulose membrane, incubated with anti-Epo antibody and peroxidase-conjugated secondary antibody, and developed with the ECL detection system. Western blot analysis of the sample containing purified rhEpo-fluorophore conjugate showed the presence of one positive band (signal). Immuno-detection of unlabeled rhEpo with the same molecular weight $(\sim 38 \mathrm{kDa})$ confirmed that the labeled molecule was rhEpo (Fig. 22B). 


\section{II.3.5 Confocal microscopy analysis of primary cultured locust brain neurons exposed to labeled rhEpo}

To study the association of rhEpo with a cell surface receptor and subsequent endocytosis, primary cultures of locust brain neurons were incubated with growth medium containing fluorescently labeled rhEpo for $10 \mathrm{~min}$ at $27^{\circ} \mathrm{C}$. Control cultures were treated in the same way, but were exposed to non-labeled rhEpo. After fixation, fixative removal and mounting, the cells were inspected with confocal microscopy.

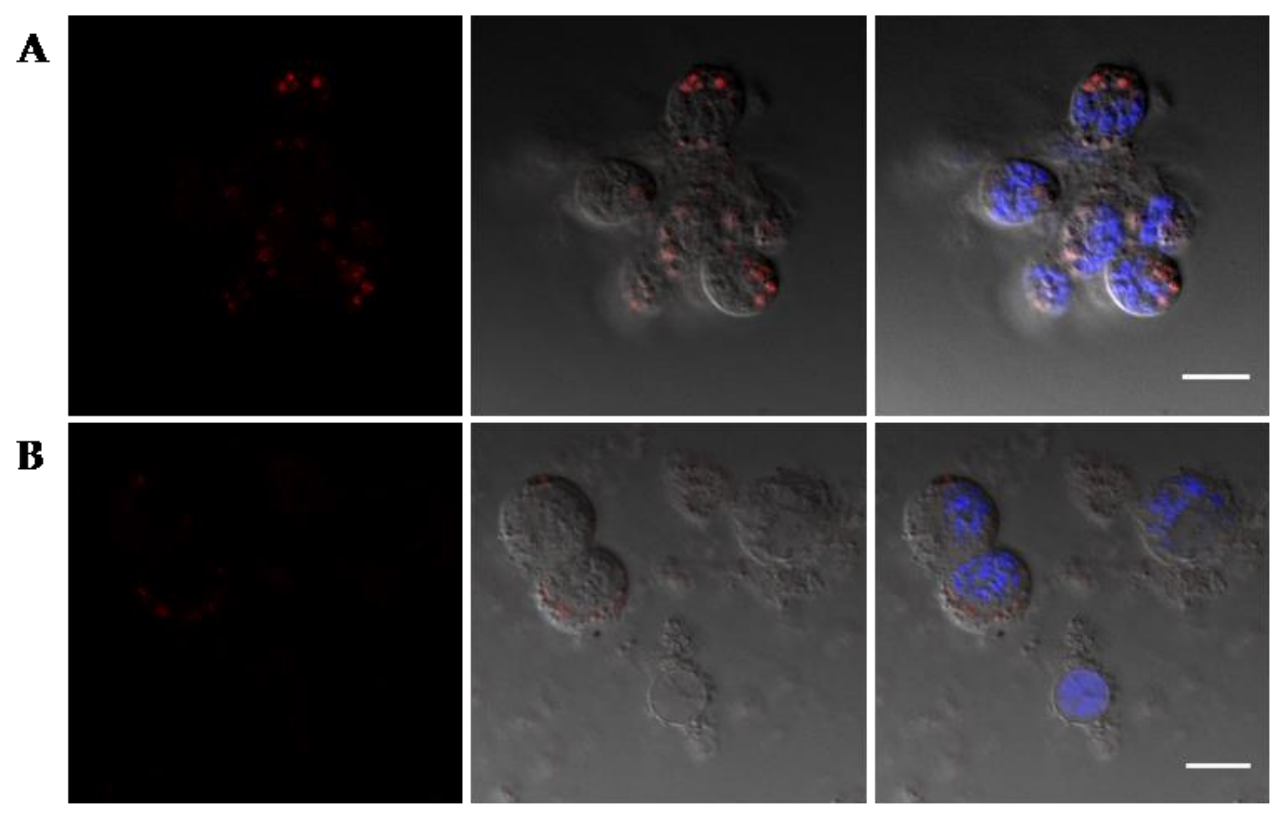

Figure 23. Visualization of receptor-mediated endocytic uptake of fluorescently labeled rhEpo by primary cultured locust neurons using confocal microscopy. Individual optical sections are presented. The fluorescence images are overlaid with differential interference contrast images of the same scanning field (middle and right). Nuclei are counterstained with DAPI (blue, right). A Neurons with fluorescently labeled vesicles (red) after being incubated with labeled rhEpo. B Neurons from control culture treated with non-labeled rhEpo without prominent vesicleassociated fluorescence. Both locust cultures, incubated with fluorescently labeled rhEpo and non-labeled rhEpo, were scanned with the same settings of the confocal microscope. Scale bars: $10 \mu \mathrm{m}$.

Cultures treated with labeled rhEpo contained neurons with fluorescently labeled vesicles of different diameters (Figs. 23A, 24A, 25). Vesicles could be clearly identified in phase contrast images. Within individual neurons only some, but not all, vesicles contained fluorescent label, suggesting that labeled rhEpo was not unspecifically incorporated with the extracellular fluid into all 
endocytotic vesicles. Furthermore, fluorescent vesicular structures were not detected in all physiologically intact cells within the culture, suggesting that only particular cell types (presumably those expressing an Epo-binding receptor) effectively incorporated labeled Epo. Control cultures occasionally exhibited background fluorescence that sometimes seemed to be associated with small cellular compartments (Figs. 23B, 24B). Such unspecific autofluorescence was sporadically detected in both, control cultures treated with non-labeled rhEpo and cultures treated with labeled rhEpo. However, cells containing weak, vesicle-associated autofluorescence were rare.

$\mathbf{A}$
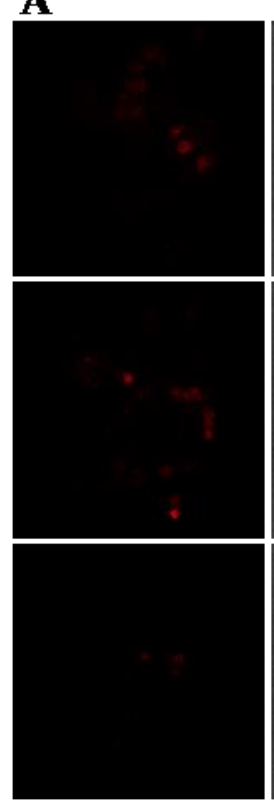
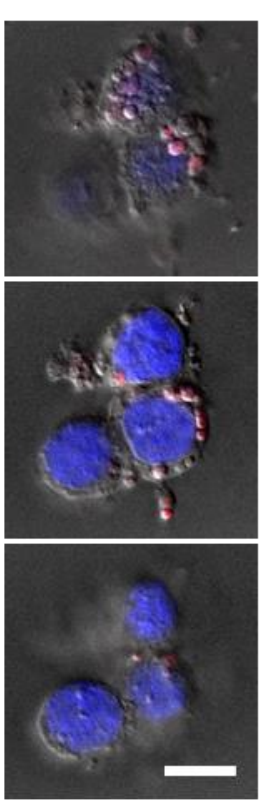

B

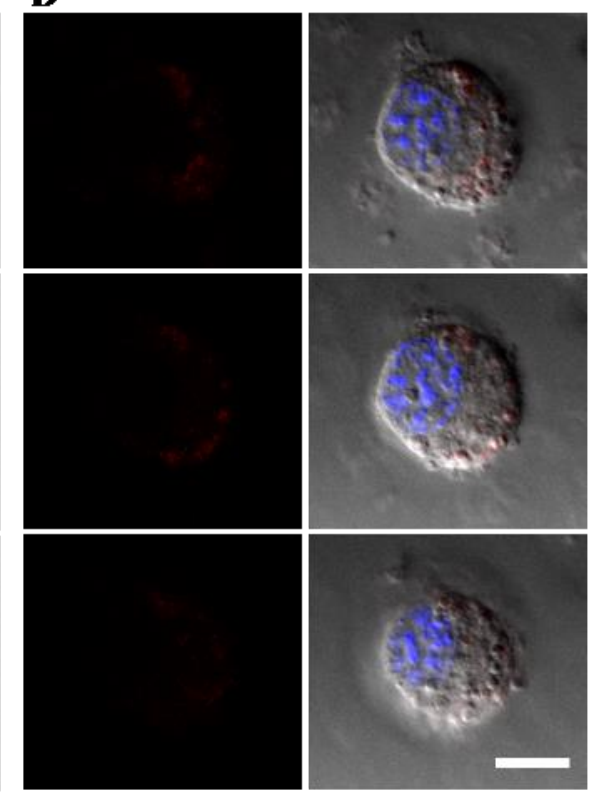

Figure 24. Three individual optical sections (upper, middle and bottom line of the panels) from one stack of primary cultured locust brain neurons incubated with fluorescently labeled rhEpo (A) and non-labeled rhEpo (B). Endocytotic vesicles possibly containing fluorescently labeled rhEpo are visualized by red fluorescence (A). Cellular autofluorescence is rather diffuse and not clearly associated with vesicles (red) (B). Nuclei are counterstained with DAPI (blue). Fluorescent images and differential interference contrast images of the same field are overlaid (right sides of the panels A and B). Scale bars: $10 \mu \mathrm{m}$.

The observations indicate that some (but not all) L. migratoria brain neurons possess the capacity to endocytose Epo, probably together with the Epo-binding receptor as a consequence of ligand-receptor binding. 

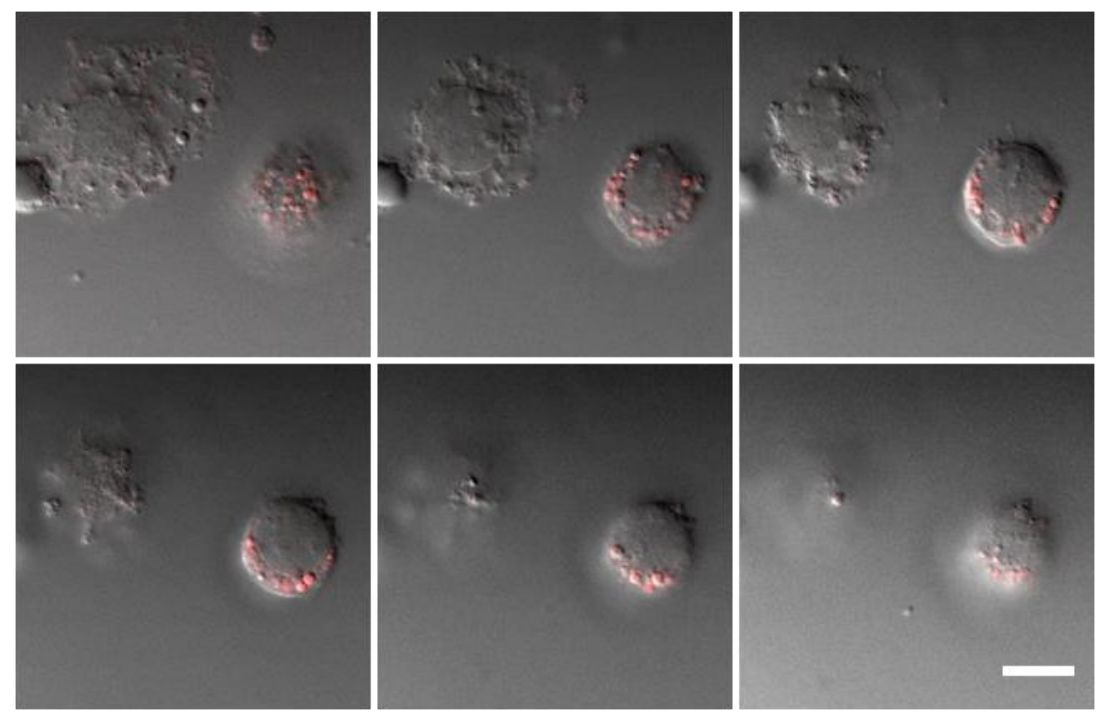

Figure 25. Six individual optical sections from one stack of primary cultured locust brain neurons incubated with fluorescently labeled rhEpo. Fluorescent images and differential interference contrast images of the same field are overlaid. Figure shows a neuron containing fluorescently labeled vesicles and a cell (possibly glia) displaying no fluorescence. Not all vesicles of the neuron that incorporated labeled rhEpo are associated with fluorescent label. Scale bar: $10 \mu \mathrm{m}$. 


\section{II.4 Discussion}

\section{II.4.1 Endocytosis of rhEpo-stimulated receptor}

The first part of this thesis characterized the transduction pathway implicated in rhEpo-mediated protection from apoptotic cell death of locust neurons in vitro. The results indicated that the receptor involved in the propagation of rhEpo's neuroprotective properties is associated with Janus kinase activity. This finding suggested that an insect orthologue of a class I cytokine receptor, which are generally associated with Janus kinases, could be responsible for the observed effects of rhEpo. The aim of the second part of my PhD thesis was to continue the functional characterization of the locust Epo-binding receptor. First, it was studied whether rhEpo is internalized and endocytosed by locust neurons in vitro, as it has been described for mammalian EpoR-expressing cells (Sawyer et al. 1987; Gross \& Lodish 2006; Becker et al. 2010). To study endocytotic vesicle formation stimulated by rhEpo, locust primary cultured neurons were exposed to FM1-43 dye. FM dyes have frequently been used to image synaptic vesicle exocytosis and endocytosis. They are specifically developed for synaptic vesicle imaging in living preparations (Gaffield \& Betz 2006). We adopted protocols from previous studies to monitor endocytosis related to stimulation with the cytokine rhEpo.

Endocytosis is a dynamic process that involves internalization of the plasma membrane along with its constituent membrane proteins and lipids. Multiple internalization processes are taking place simultaneously at the cell surface. Through these processes cells are sampling information about their extracellular milieu, which is essential for their proper functioning (Kumari et al. 2010), and adjust the presence of functional membrane proteins to the physiological state. As a consequence of constant sampling and metabolite uptake from the medium, we observed a basal rate of endocytosis in both unstimulated control and rhEpo-stimulated neuronal cultures. Previous studies also demonstrated that receptors can be internalized in the absence of their ligands (Hémar et al. 1994; Basquin \& Sauvonnet 2013). Ligand-independent 
endocytosis of cytokine receptors plays a role in receptor turnover, a complex mechanism that regulates the number of receptors present on the cell surface available for interaction with ligands. This mechanism was shown to be important for information processing of a broad range of cytokine concentrations. It especially applies to cells expressing erythropoietic EpoR, which is generally expressed on external cellular membranes in very low copy numbers (Becker et al. 2010).

Several mechanisms of endocytosis have been described in mammals. Receptormediated endocytosis through clathrin-coated pits and vesicles is especially well characterized (McMahon \& Boucrot 2011; McPherson et al. 2000). Using a "flash-and-freeze" approach, it was recently revealed that endocytosis of vesicles at presynaptic membranes under physiological conditions is an extremely fast process and most likely clathrin-independent (Watanabe et al. 2013a; 2013b). Clathrin-independent endocytosis induced by cytokines has also been identified (Lamaze et al. 2001). In all forms of endocytosis the formation of vesicles critically depends on the activity of actin and dynamin. Most aspects of receptor-mediated endocytosis, particularly those requiring intimate contact with actin and other cytoskeletal elements, seem to be conserved across phyla (Galletta \& Cooper 2009). In D. melanogaster, the genes that are implicated in crucial steps of vesicle formation and transport, dynamic cytoskeletal organization and protein sorting pathways have been identified and most of them are orthologues of vertebrate genes involved in identical functions (reviewed in: Fischer et al. 2006; Narayanan \& Ramaswami 2001; Li et al. 2015). Some examples are the Drosophila orthologues of mammalian clathrin coated pit components, such as clathrin heavy chain (Bazinet et al. 1993), dynamin forms (coded by shibire gen) (Chen et al. 1991), alpha-adaptin (Dornan et al. 1997) and beta-adaptin (Camidge \& Pearse 1994).

„Flash and freeze“ studies on synaptic vesicles also provided a satisfying answer to often contradictory observations obtained from studies on the kinetics of endocytosis in mammalian cells. While clathrin-independent fast 
endocytosis requires higher (mammalian typical) temperatures, clathrinmediated endocytosis also takes place at lower temperatures (Watanabe et al. 2013b). Without specifying the endocytosis mechanism that led to vesicle formation in locust neurons we observed a basal endocytotic activity that was independent of rhEpo stimulation.

Our results clearly demonstrated that, in addition to the basal endocytotic activity, application of rhEpo stimulated endocytosis in primary cultured locust brain neurons. Two different approaches were used to evaluate the same set of data from experiments with the dye FM1-43. The observer-based method selected physiologically intact neurons, using nuclear DAPI staining as a criterion, for subsequent quantification of endocytotic vesicles. Since FM1-43associated fluorescence was only inspected after choosing regions with a sufficiently high number of intact neurons, a bias to select neurons with particular amounts of endocytotic vesicles for the analysis was excluded. In contrast, the unsupervised software-based evaluation of the same confocal scans selected cells for evaluation on the basis of round shape, using criteria defined by an algorithm to exclude irregularly-shaped and presumably compromised single cells and cells whose borders overlapped from the analysis. Both methods, observer-based and unsupervised software-based evaluation, selected different populations of cells for endocytotic vesicle quantification from the same confocal images. Additionally, endocytosis was quantified in two different ways. In the observer-based evaluation, vesicles were counted for each neuron. Reliable counting was only possible for up to 15 vesicles per neuron since higher numbers of vesicles per cells could not be clearly separated for individual recognition. Therefore, neurons were categorized into four groups containing ranges of labeled endocytotic vesicles. Overlap of individual vesicles in confocal scans was not problematic in the software-based evaluation, since in this case the total volume of labeled vesicles per individual neuron was determined. Despite the differences in the selection of evaluated neurons and the measured parameters (numbers versus total volume of endocytotic vesicles) both ways of data analysis documented a 
significant stimulatory effect of rhEpo on the endocytotic activity during both incubation times tested (2 and $10 \mathrm{~min}$ ). Hence, similar to mammalian homodimeric EpoR of erythrocyte progenitor cells, the tissue-protective Epobinding receptor on locust brain neurons is also endocytosed upon ligand binding. To my knowledge, this has not been shown in any non-hematopoietic cell type.

\section{II.4.2 Chemical labeling of rhEpo to study its endocytosis after binding to its receptor}

Following binding to a membrane receptor, Epo stimulates endocytosis of locust neurons. The destiny of the Epo-receptor complex remains unknown. There are multiple possibilities. Epo could be degraded by extracellular enzymes. For instance, dipeptidyl peptidase-4 that is expressed on the surface of most cell types cleaves various cytokines, including Epo, within N-terminal regions. This truncation typically decreases subsequent cytokine activity (Broxmeyer 2013). Alternatively, Epo could be internalized together with its receptor (Sawyer et al. 1987). If internalized, sorting mechanisms may direct both Epo and its receptor to degradation in lysosomes, as demonstrated in mammals (Gross \& Lodish 2006; Neumann et al. 1993; Walrafen et al. 2005) or lead to re-introduction of recycled receptor into the cytoplasmic membrane.

Different approaches can be used to study ligand-receptor binding. Traditionally, receptor-ligand interactions have been monitored using radiolabeled ligands. However, there are multiple drawbacks to the use of radioactivity, including production, delivery, and disposal of the radioactive materials, relatively short shelf life, and the long signal acquisition times required to reach the desired sensitivity (Handl et al. 2005). Fluorescence-based binding assays emerged as promising alternatives to radioactive assays. Direct measurement of fluorescent intensity of ligand bound to receptor-containing membranes presents probably one of the simplest approaches to study ligandreceptor interaction (Kubala et al. 2004). 
The sensitivity of binding assays critically depends on the number of cell surface receptors and their affinity for the labeled ligand. Epo-binding receptors generally have low expression levels on the cell surface, making it difficult to distinguish receptor-bound ligand from unspecific association with the cell membrane. Human Epo-responsive erythroblasts for instance exhibit only 1001000 cell-surface EpoR per cell (Broudy et al. 1991) and the number of tissueprotective Epo receptors expressed on the surface of non-hematopoietic cells is thought to be even lower (Um \& Lodish 2005; Sanchez et al. 2009; Elliott et al. 2014).

Human Epo is a single polypeptide of 166 amino acids (Lin et al. 1985; Jacobs et al. 1985) containing four antiparallel amphipathic a-helices (A,B, C and D) connected by loops $(\mathrm{AB}, \mathrm{CD}$ and $\mathrm{BC})$ that lack a particular secondary structure (Lai et al. 1986). From its primary structure it was predicted that four antiparallel a-helices form a globular three-dimensional structure that is stabilized by two disulphide bridges between cysteines 6 and 161 and between cysteines 29 and 33 (Bazan 1990; Boissel et al. 1993). This prediction was confirmed by nuclear magnetic resonance microscopy of Epo in solution (Cheetham et al. 1998) and by X-ray crystallographic analysis of Epo bound to EpoR (Syed et al. 1998). The primary structure of rhEpo was shown to be identical to that of the endogenous hormone except for the in vivo posttranslational cleavage of an arginine at the carboxy-terminus, resulting in a 165 amino acid long polypeptide. The protein content of endogenous hormonal human Epo and rhEpo contributes approximately 60\% to their total molecular mass of approximately $30.4 \mathrm{kDa}$ (Lai et al. 1986; Bunn 2013). The remainder consists of carbohydrates that include one O-linked and three N-linked oligosaccharide side chains in human Epo molecules. The carbohydrates are not required for receptor binding but increase the half-life of the circulating hormone (Tsuda et al. 1990; Takeuchi et al. 1990). Eight lysine residues are contained within the primary structure of Epo. Together with one free aminoterminus, nine amino groups per rhEpo molecule are available for a chemical reaction with the NHS-fluorophore complex. The efficiency of protein labeling, 
however, depends critically on the number of primary amino groups that are solvent accessible (Patil et al. 2013). The NMR structure of human Epo revealed that Lys 45 for example is located in a solvent-accessible position at the $\mathrm{D}$ helix/ AB loop interface. Due to the kink that is introduced in the D helix at the position Gly 151, Lys 152 is brought into hydrophobic contact with Val 63, Trp 51, and Phe 148 and is therefore most likely not readily solvent accessible for chemical modification (Cheetham et al. 1998). However, after the labeling reaction and subsequent removal of free dye, the determined degree of labeling $(\mathrm{DOL}=1.4)$ suggested that on average only one to two fluorophore molecules have been cross-linked to each rhEpo molecule. Fluorescent scanning at 650nm of the SDS-polyacrylamide gel containing the labeled rhEpo sample showed a single fluorescent band at the expected molecular weight for rhEpo confirming that rhEpo has been labeled. As there were no other fluorescent signals detected in the sample, free dye must have been effectively removed and other fluorescent contaminants were not present. The same gel was afterwards used for protein transfer onto a nitrocellulose membrane and immunoreaction with anti-Epo antibodies. Western blot analysis confirmed the existence of only one immunoreactive band in the sample, with the expected molecular weight of rhEpo. RhEpo that was subjected to NHS ester reaction of labeling had approximately the same size as non-labeled rhEpo.

Even though the usage of fluorescent probes is relatively safe compared to radioactive probes and fluorescently labeled proteins could be easier implemented into ligand-receptor binding assays, fluorescent ligand-based binding assays have two main drawbacks. Cross-linking of the fluorophore to the polypeptide may add steric hindrance as well as hydrophobic modifications which can significantly alter the pharmacological properties of the ligand. Its affinity may be diminished to such an extent that it cannot be used for pharmacological studies (Cottet et al. 2011). However, protein carbamylation, a reaction that leads to the conversion of all lysine residues into homocysteines, led to the generation of an Epo derivative (cEpo) that had lost its erythropoietic 
properties, but retained tissue-protective activity (Leist et al. 2004). Similarly to carbamylation, the labeling reaction used in our study typically targets lysine residues, implying that labeled Epo will not necessarily lose its tissue-protective properties. Since on average only two of nine possible sites have been altered by the labeling reaction the overall structure of rhEpo is expected to be only slightly changed. However, whether labeled rhEpo retains its ability to protect locust brain neurons has not yet been tested.

The second drawback that makes the implementation of fluorescence-based ligand-receptor binding assays difficult, is the background signal that can result from autofluorescence of biological tissues (Kubala et al. 2004). Biomolecules that have intrinsic fluorescence tend to autofluoresce under the same illumination conditions used for the excitation of the fluorophore-ligand conjugate. The main sources of autofluorescence in mammalian cells are nicotinamide adenine dinucleotide phosphates (NADPH) at wavelengths between 400 and $500 \mathrm{~nm}$ and flavins and flavoproteins at wavelengths between 500 and $600 \mathrm{~nm}$ (Knight \& Billinton 2001). One challenge for successful detection of fluorescently labeled molecules at the cellular level is to enhance the signal-to-noise ratio. This can be achieved by decreasing the intrinsic cellular fluorescence background or increasing the intensity of the specific fluorescent signal (Handl et al. 2005). To avoid excitation of NADPH and flavins, a fluorophore (NT-647) that is excited by and emits light in the red portion of the spectrum was used, with the excitation maximum at $630 \mathrm{~nm}$ and emission maximum at $650 \mathrm{~nm}$. Typically, longer excitation wavelengths contain too little energy for the excitation of shorter wavelength-emitting molecules and hence cause lower intensities of background fluorescence. Still, primary cultured locust brain cells occasionally emitted autofluorescence. Careful analysis showed that this unspecific autofluorescence can be distinguished from the fluorescence of vesicles containing labeled rhEpo.

In contrast to untreated control cultures, neurons exposed to labeled rhEpo contained fluorescently labeled vesicles. Fluorescence was exclusively 
associated with vesicles, which were identified by differential interference contrast scans that were overlaid with fluorescent images. Importantly, not all cells in the cultures treated with labeled rhEpo displayed vesicle-associated fluorescence. Hence, only certain neurons had the ability to internalize labeled rhEpo. This suggests that the Epo-binding receptor is expressed in the cellular membranes of some, but not all, locust neurons. Furthermore, in neurons that incorporated labeled rhEpo not all vesicles contained the fluorescent label. This indicates that even though constitutive endocytosis occurs simultaneously, only the endocytosis initiated upon binding of labeled rhEpo to a specific receptor will lead to the formation of vesicles containing labeled rhEpo. Thus, the detection of fluorescence in the vesicles of locust neurons exposed to the labeled rhEpo provides additional evidence that labeled rhEpo is endocytosed by locust neurons as a consequence of binding to an unidentified receptor.

Upon binding to the classical homodimeric EpoR and subsequent endocytosis of the ligand/receptor complex, Epo is degraded in lysosomes (Gross \& Lodish 2006). Ubiquitination at the membrane-proximal cytoplasmic domain of EpoR is required for efficient Epo-induced internalization and lysosomal degradation (Gamze et al. 2011) and thereby regulates the temporal characteristics of intracellular signaling (Mayuzumi 2010; Gamze et al. 2013). Whether endocytosed insect Epo-binding receptors are degraded or recycled and relocated to the plasma membrane cannot be deduced from our experiments. A chase in growth medium without ligand for different periods of time following endocytosis of labeled rhEpo may provide information about the temporal and spatial distribution of internalized labeled rhEpo. For instance, if the cells become depleted of internalized labeled rhEpo after a chase, this would indicate that the ligand/receptor complex is recycled to the plasma membrane and the ligand resecreted. Decreased numbers of labeled vesicles after a chase and larger sizes of remaining vesicles in comparison to the size of vesicles detected immediately after incubation with labeled ligand may indicate the maturation of endosomes into lysosomes and subsequent degradation of labeled ligand. 


\section{II.4.3 Protective effects of the non-erythropoietic Epo variant EV3 on the survival of primary cultured locust brain neurons challenged with hypoxia}

Many derivatives of Epo exert neuroprotection without stimulating erythropoiesis. Therefore, homodimeric EpoR that stimulates erythropoiesis and Epo-binding receptors that mediate neuroprotection in vertebrates must have structural and functional characteristics that allow differential activation by different ligands and the separation of erythropoietic from cytoprotective functions. Although multiple transcription start sites that could be used differentially in the liver, kidney, and brain in response to anemia have been identified in the human epo gene (Semenza et al. 1990), until recently alternatively spliced forms were not detected in human organs. A brain-specific alternatively spliced transcript of the epo gene was detected in a teleost fish, the puffer fish Fugu rubripes, whose erythropoietic Epo molecule shares around 32\% homology with human Epo. It remains to be determined whether this brainspecific epo transcript, that includes an alternate first exon, has properties different from those of the erythropoietic Fugu Epo variant (Chou et al. 2004). During her PhD thesis Christel Bonnas discovered several alternative splice variants of the murine and human epo gene. A variant of human Epo (EV3) that was isolated from fetal human brain did not stimulate erythropoiesis in mice. Similar to many other Epo derivatives the EV3 variant mediated cytoprotective effects, including neuroprotection, in different mammalian in vitro systems (Bonnas 2009).

Alternative splicing, the inclusion of different exons into mRNA, leads to the generation of different protein isoforms from a single gene, permitting new functions to be established without eliminating the original function of a protein. Not all of alternatively generated transcripts are functional on the protein level. It has been proposed that selection against deleterious isoforms prevents their increase in frequency and assures their eventual disappearance from the gene pool, while inert isoforms that can be tolerated by cells might eventually acquire new functions which may provide a future evolutionary advantage to the organism. Once a new functional transcript is established, the 
frequency of its inclusion into a proteome of the species increases (Keren et al. 2010). While more than $90 \%$ of human genes undergo alternative splicing (Wang et al. 2008), the frequency of alternatively spliced genes is rather low in insects (Kim et al. 2007). Unlike most insect species exceptionally large introns have been detected in locusts contributing to the large size of their genome (Wang et al. 2014). Locusts seem to share some characteristics of their introns with vertebrates, rather than with other insects, such as the abundance of U12type introns (minor-class intron) and the ratio of ratcheting point sites (Wang et al. 2014), indicating the possibility of convergent evolution of splicing mechanisms associated with genome size expansion in animals.

We hypothesized that, if a tissue-protective receptor in locust neurons is evolutionarily closer related to the mammalian tissue-protective nonhematopoietic receptors than to mammalian erythropoietic EpoR, the human splice variant EV3 should support the survival of locust neurons exposed to apoptosis-inducing stimulus. Similar to neuroporotective properties of rhEpo observed in an oxygen-glucose deprivation model studied in mice (Bonnas 2009), the EV3 variant showed a dose-dependent positive effect on the survival of hypoxia-challenged locust neurons, with a concentration optimum around $3.33 \mathrm{ng} / \mathrm{ml}$ (corresponding to $\sim 0.4 \mathrm{U} / \mathrm{ml}$ ), and with ten times lower and higher concentrations being less effective. Even though only one experiment was performed with a wider concentration range of EV3 (from 0.33 to $33 \mathrm{ng} / \mathrm{ml}$ ), it provided an approximate estimation of the concentration range being most effective for protection of primary cultured locust neurons from hypoxiainduced apoptotic cell death. The estimated optimum concentration was confirmed in the next series of experiments with three different concentrations of EV3 covering a narrower range around this estimated optimum, namely 2.08, 4.17 and $8.33 \mathrm{ng} / \mathrm{ml}$. These concentrations were applied in comparison with 4 $\mathrm{U} / \mathrm{ml}$ rhEpo, which has previously been shown to be most beneficial in protecting locust brain neurons from apoptosis induced by hypoxia (Ostrowski et al. 2011). In all three tested concentrations EV3 showed protection of locust neurons, with at least equal potency as rhEpo. 
With respect to EV3 binding the locust receptor responsible for transducing neuroprotective effects is more similar to the mammalian non-hematopoietic tissue-protective receptor, than to the classical homodimeric erythropoietic EpoR of mammals. Whether the same receptor type is involved in the propagation of neuroprotective properties of rhEpo and EV3 in locusts and other species, as it could be assumed, remains to be studied. This could be achieved by demonstrating that EV3 initiates the same intracellular transduction pathways or in studies showing that the stimulating effect of Epo on endocytosis could be suppressed by preincubation with EV3 and vice versa.

Table 1. Comparison of characteristics of Epo-binding receptors in invertebrates and vertebrates.

\begin{tabular}{|l|c|c|c|}
\hline & $\begin{array}{l}\text { Tissue-protective } \\
\text { locust receptor }\end{array}$ & $\begin{array}{l}\text { Tissue-protective } \\
\text { vertebrate receptor }\end{array}$ & $\begin{array}{l}\text { Hematopoietic } \\
\text { vertebrate EpoR }\end{array}$ \\
\hline $\begin{array}{l}\text { Association with } \\
\text { JAK-STAT }\end{array}$ & + & + & + \\
\hline $\begin{array}{l}\text { Epo-stimulated } \\
\text { endocytosis } \\
\text { of receptor }\end{array}$ & + & $?$ & + \\
\hline $\begin{array}{l}\text { Activation } \\
\text { by EV3 }\end{array}$ & + & + & - \\
\hline
\end{tabular}

\section{II.4.4 Pre-vertebrate evolution of a tissue-protective ligand-receptor system with structural and functional similarity to the mammalian Epo/Epo-receptor system}

We characterized two properties of insect (Locusta migratoria) Epo-binding neuroprotective receptors: (1) Endocytosis of the receptor following stimulation with rhEpo, similar to mammalian homodimeric EpoR; (2) Activation of the receptor by the Epo splice variant EV3, which does not stimulate erythropoiesis (Table 1). Our results suggest that Epo-binding receptors involved in mammalian and insect neuroprotection have evolved from a common ancestral prototype that was later, after vertebrates evolved, adapted for the regulation of 
red blood cell production. Since both neuroprotective and erythropoietic Epo receptors typically transduce the information of ligand binding into cytoplasmic signals through JAK/STAT transduction pathways, their common predecessor was presumably a member of the type I receptor superfamily, which also includes the classical EpoR.

Type I cytokine receptors are characterized by cytokine receptor homology domains (CHD) in the extracellular region containing a conserved WSXWS motif that is required for interaction with the ligand. They all bind ligands with a spatial „four a-helix bundle“ organization and employ intracellular signaling molecules of the JAK and STAT families (Boulay et al. 2003). Despite these common structural features, type I cytokine receptor chains share little primary sequence homology (Bazan 1990). Their structural relatedness was deduced from similar intron/exon structures and gene clustering observed for certain cytokine and cytokine receptor families, rather than from sequence alignment approaches (Boulay \& Paul 1992). Individual type I cytokine receptor chains assemble into functional receptor complexes by forming either homodimers or various heterocomplexes, that bind one or multiple specific ligands (Liongue \& Ward 2007). Due to their tendency to form different heterocomplexes, type I cytokine receptors are often activated by several ligands. Individual cytokines typically activate multiple receptor complexes, which contributes to functional redundancy of this receptor superfamily (Ozaki \& Leonard 2002). Concordantly, a number of structurally different Epo derivatives have been demonstrated to activate the mammalian tissue-protective Epo receptor without stimulating erythropoiesis. Among them, a 11 amino acid long peptide, that was synthesized to mimic a particular feature of Epo's three-dimensional structure, has a primary sequence completely unrelated to Epo's primary sequence (Brines et al. 2008). These observations imply that the spatial organization of a few critical amino acid residues of a ligand, rather than its primary amino acid sequence is the crucial factor for its differential binding to the tissue-protective receptor. 
Although epo and epor gene have been identified in vertebrate species, from fish (Chu et al. 2008) to mammals (Wen et al. 1993), alignment of nucleotide sequences of several insect species (Drosophila melanogaster, Tribolium castaneum, Gryllus bimaculatus and Manduca sexta) with Epo and EpoR sequences from vertebrates (Mus musculus, Xenopus tropicalis and Danio rerio) did not reveal any sequence similarity to identify potential insect orhologues of vertebrate Epo and EpoR (Hahn 2014). Similarities in amino acid sequence of human Epo protein and deduced amino acid sequences of Epo proteins from other mammals range from 80 to $91 \%$ (Wen et al. 1993; Suliman et al. 1996) while Epo proteins from the puffer fish Fugu rubripes (Chou et al. 2004) and the zebrafish Danio rerio (Chu et al. 2007) share an overall identity of only $32 \%$ with human Epo protein. Interestingly, two years before the Fugu epo gene was cloned and characterized, a homology search of the Fugu genome using protein sequence of human Epo as a query revealed no orthologues of human Epo, misleading to the conclusion that Epo is either absent in fish or has evolved rapidly since the divergence of the mammalian and fish lineages (Aparicio et al. 2002). Hence, a genome or protein sequence comparison across phyla may be the easiest, but not the most accurate method for the identification of potential insect orthologues of human tissue-protective peptides and their receptors.

Tissue-selective effects of Epo and Epo derivatives together with differences in ligand binding affinities (Collino et al. 2015) suggested that alternative receptor complexes are mediating tissue protection in non-hematopoietic tissues. For instance, a heterodimer composed of one EpoR monomer and $\beta c$ chain has been shown to be involved in the protection of murine cardiomyocites and murine spinal cord neurons (Brines et al. 2004). $\beta c$ chain is a non-specific signaltransduction chain that contributes a receptor subunit to interleukin-3 receptor, interleukin-5 receptor and granulocyte-macrophage colony stimulating factor receptor converting the low affinity interaction between ligand and a-chain of these receptors to higher affinity (Scott \& Begley 1999). Another signal transducer that is a commonly shared component of many interleukin-6 family receptors, such as oncostatin $\mathrm{M}$ receptor (OSMR) and leukemia inhibitory factor 
receptor (LIFR), is archetypal glycoprotein 130 (gp130). Gp130 is highly conserved among vertebrates and is thought to represent an ancestral form of type 1 cytokine receptors (Boulay et al. 2003; Liongue \& Ward 2007). A heteromeric receptor complex consisting of an a-chain of LIFR and gp130 chain is involved in EV3-mediated protection of primary cultured rat cortical neurons challenged by glucose and oxygen deprivation (Bonnas 2009). Another tissue protective receptor, including the ephrine B4 receptor, either in a homodimeric form or a heterodimer associated with one EpoR molecule, has also been proposed and patented (Jackson et al. 2012; Debeljak et al. 2014). Ephrine receptors are single-pass transmembrane proteins with highly conserved extracellular and intracellular domains and belong to the family of receptor tyrosine kinases (Yamaguchi \& Pasquale 2004). Ephrine B4 receptor has been demonstrated to mediate survival signals in cancer cells (Jackson et al. 2012). Finally, the orthologues of human orphan receptor, cytokine receptor like factor 3 (CRLF3), identified in several insect species, but not in Drosophila melanogaster (Wyder et al. 2007; Hahn 2014) are promising candidates for the binding partners of unidentified tissue-protective receptors present in invertebrate and vertebrate species in which Epo-mediated neuroprotection has been demonstrated. CRLF3 essentially consists of just a CHD domain, a minimal domain required for the classification into type 1 receptor superfamily. The high conservation of CRLF3 across vertebrate and invertebrate species indicates an essential conserved function for this protein (Liongue \& Ward 2007). Hence, differential tissue-protective responses are likely mediated by different receptor complexes, some of them formed by yet unidentified partners.

Invertebrates and vertebrates possess common mechanisms underlying innate immunity. However, unlike vertebrates, invertebrates lack acquired immunity (Rowley \& Powell 2007). It has been hypothesized that type I cytokines and their receptor chains involved in pre-vertebrate innate immune functions adopted new functions, in addition to their original function during the emergence of adaptive immunity in the vertebrate lineage (Ozaki \& Leonard 2002; Boulay et al. 2003; Buchmann 2014). Although the function of cytokines in 
insect innate immunity has not been intensively studied, molecules similar to vertebrate cytokines, cytokine receptors and intracellular pathways they activate, have been identified in insects. These include cytokine-like proteins that have been functionally characterized in D. melanogaster, such as for example Spaetzle, the Drosophila ligand for Toll receptor (Weber et al. 2003), Unpaired (Agaisse et al. 2003), which is closely related to the vertebrate leptins (Boulay et al. 2003), and Eiger, the Drosophila TNF orthologue (Mabery \& Schneider 2010). In addition, many insect peptides, such as sex peptide (Peng et al. 2005), growth-blocking peptide (GBP) (Hayakawa 1991), hemocyte chemotactic peptide (Nakatogawa et al. 2009), plasmocyte spreading peptide (Strand et al. 2000) and stress-responsive peptide (Yamaguchi et al. 2012) are acknowledged as cytokine-like factors. Corresponding insect cytokine-like receptors have also been reported, including the Dome protein from $D$. melanogaster (Brown et al. 2001), growth-blocking-peptide receptor (Watanabe et al. 2006) and the putative receptor for plasmocyte spreading peptide (Clark et al. 2004). In vertebrates, cytokines are common mediators of immune-neural interactions (Maier 2003) and cytokine-like molecules in insects may play a similar role (Adamo 2008). For example, GBP, which is believed to be an insect orthologue of vertebrate EGF, was found in both immune cells and glial cells of the CNS (Hayakawa \& Noguchi 1998). Genes known to be involved in insect immunity have also been reported in locusts (Wang et al. 2014).

In summary, functional similarity of the receptor that mediates neuroprotective properties of Epo in locust neurons with the mammalian neuroprotective Epo receptor is reflected by the fact that both receptors are activated by the nonhematopoietic Epo splice variant EV3. In addition, both receptors transduce neuroprotection via the evolutionary conserved JAK/STAT intracellular pathway. This suggests that the evolutionary origin of Epo-like signaling might have been related to tissue protection. Subsequent evolution has shaped the ancestral pre-vertebrate ligand/receptor pair as an answer to different selection pressures that different lineages had to encounter. In the vertebrate lineage this system has acquired an additional function in the regulation of erythropoiesis. 
The importance of this system is underlined by the fact that though it might have evolved in different directions, it still retained the ability to mediate what is most important for a cell (and for the whole organism) - an adaptive selfpreserving response to unfavorable conditions or harmful stimuli. 


\section{Potential effects of erythropoietin on insect neurogenesis}

\section{III.1 Introduction}

The best characterized role of Epo is the control of red blood cell production that takes place within fetal liver and adult bone marrow of mammals (Jelkmann 2013). Red blood cells are formed continuously from pluripotent hematopoietic stem cells via generation of erythroid progenitors. The earliest erythroid progenitors are slowly proliferating burst-forming unit-erythroids (BFU-E), which are not responsive to Epo and do not express the EpoR. They develop into mature BFU-E that start to express EpoR and are weakly responsive to Epo. These cells give rise to late erythroid progenitors, colony forming units-erythroids (CFU-E). CFU-E are highly responsive to Epo and develop further into erythroblast colonies (Wu et al. 1995). Thus, Epo and EpoR are crucial for the proliferation and salvaging of late erythroid progenitors CFU-E from apoptosis and their irreversible terminal differentiation into erythroblasts (Youssoufian et al. 1993). During maturation of erythroblasts, the number of EpoR per cell decreases. Beyond the late basophilic erythroblast stage the cells are no longer dependent on Epo for continued maturation (Koury \& Bondurant 1988).

Apart from its effect on erythroid progenitors, Epo has the capacity to stimulate the production of neural stem cells (NSC) within embryonic and adult mammalian brains. Both Epo and EpoR are expressed in the nervous system of mammals during embryonic development (Liu et al. 1997; Juul et al. 1998; Juul et al. 1999), suggesting that Epo signaling is required for normal brain development. Although their expression levels decline once the brain development has been completed, expression of Epo and EpoR is upregulated in the adult brain upon injury (Digicaylioglu et al. 1995; Marti et al. 1996; Morishita et al. 1997; Brines et al. 2000; Bernaudin et al. 2000; Sirén et al. 2001b) 
and constitutes an integral part of the intrinsic host response mediating tissue protection and regeneration (Brines \& Cerami 2005). Epo-mediated support of tissue repair after injury is accomplished, at least in part, via the ability of Epo to enhance the NSC production (Shingo et al. 2001) and promote migration and differentiation of progenitor cells (Hassouna et al. 2016). Infusion of an antimitotic agent, which inhibits neurogenesis, abolished Epo-mediated functional recovery in a rat traumatic injury model (Zhang et al. 2012). Epo has been reported to enhance adult hippocampal neurogenesis in rodents under both, basal conditions (in mice) (Ransome \& Turnley 2007; Hassouna et al. 2016), and following traumatic brain injury (in rats) (Lu et al. 2005). This property of Epo most likely contributes to improved cognitive performance observed in rodent models of neurological and psychiatric disease (Adamcio et al. 2008; Sargin et al. 2011) and in patients with treatment-resistant depression (Miskowiak et al. 2014) and schizophrenia (Kästner et al. 2012). In a rodent model of stroke Epo enhanced neurogenesis in the subventricular zone (SVZ) and increased neuroblast migration to areas exposed to ischemic damage in vivo (Wang et al. 2004). Furthermore, mice with brain-specific deletion of EpoR have a proliferation deficit in the adult SVZ and decreased neurogenesis (Tsai et al. 2006). In addition to its influence on neuronal progenitors, Epo and the Epo derivative asialo-erythropoietin promote the differentiation of oligodendrocyte progenitor cells into mature oligodendrocytes both, in in vitro (in prenatal rat) (Jantzie et al. 2013), and in vivo (in neonatal mice) (Kako et al. 2012) rodent models of white matter injury.

The role of Epo in adult genesis of neuronal and oligodendroglial progenitors from NSC is in agreement with the hypothesis of Epo's original function in tissue protection. As a more general "signal“, Epo promotes healing and recovery of injured tissue not only via engagement of anti-apoptotic cellular mechanisms (to protect cells that have not been severely injured), but as well through stimulation of NSC proliferation, their migration to the site of injury and differentiation into new neurons and oligodendrocytes, to replace the cells that have been lost as a consequence of injury. Additionally, to facilitate the 
migration of NSC, Epo stimulates remodeling of the cerebral vasculature (Wang et al. 2006b). Therefore, as a "master regulator" of wound and injury (Brines 2014), Epo can influence an array of host responses designed to promote a comprehensive defense against harmful stimuli.

The aim of the third part of the thesis was to study whether Epo plays a role in insect neurogenesis. To study this question a prerequisite was the availability of a suitable model. Three different model systems have been assessed. A permanent cell line derived from tobacco hornworm moth Manduca sexta embryonic tissue (Eide et al. 1975) has been chosen as a first model system for in vitro studies, due to its proliferative capacity. Conditioned medium from the $M$. sexta cell line has been used previously as a medium supplement to support survival of primary cultured olfactory receptor neurons from $M$. sexta pupae (Stengl \& Hildebrand 1990). Given that Epo-mediated neuroprotection has been demonstrated in Locusta migratoria, I wanted to test furthermore whether primary cultures established from nymphal optic lobes of the same species can be applied for studying a potential neurogenic effect of Epo. Neurogenesis taking place in the optic lobes has been demonstrated in adult moths (Dufour \& Gadenne 2006) and recently in adult Drosophila melanogaster (FernándezHernández et al. 2013). Finally, to analyze the ability of Epo to stimulate adult insect neurogenesis, the suitability of red flour beetle Tribolium castaneum for in vivo studies has been examined. Adult neurogenesis has been found in several insect species (Cayre et al. 1996). In adult insects neurogenesis occurs predominantly in the mushroom bodies. This part of the insect brain represents a main higher-order sensory integration center that plays a key role in olfactory learning and memory (Cayre et al. 2007). T. castaneum emerged recently as a promising model for studies of the cellular and molecular regulation of adult neurogenesis, since in this species mushroom body neuroblasts continuously proliferate during the first two months of adult life (Zhao et al. 2008). This capacity could be of great importance from an evolutionary perspective, given that in most insect species neurogenesis seems to be restricted to embryonic and early pupal development (Ito \& Hotta 1992; Cayre et al. 1996). 


\section{III.2 Material and Methods}

\section{III.2.1 Manduca sexta cell line}

\section{III.2.1.1 Manduca sexta cell line maintenance}

The cell line was established from embryonic tissue of tobacco hornworm moth (Eide et al. 1975). The cell line was maintained in cell culture flasks $\left(25 \mathrm{~cm}^{2}\right.$, Orange Scientific, Braine-l'Alleud, Belgium) filled with Grace's Insect Medium (Gibco, Life Technologies, Thermo Fisher Scientific, Braunschweig, Germany) supplemented with 10\% FBSG (PAA Laboratories, GE Healthcare Life Sciences, Freiburg, Germany) (Grace's/10\%FBSG) at $20^{\circ} \mathrm{C}$ in an incubator (Heratherm, Thermo Fisher Scientific). Cells were passaged every 2 weeks together with the replacement of the medium. For passaging, the old medium was removed, cells washed once with phosphate-buffered saline (PBS) and then detached from the flask surface by mechanical pressure applied through a Pasteur pipette filled with fresh Grace's/10\% FBSG medium. The resulting cell suspension was collected, its amount reduced by half and replated into new flasks. Flasks with replated cells were filled to a volume of $10 \mathrm{ml}$ with fresh Grace's/10\% FBSG and kept in incubator at $20^{\circ} \mathrm{C}$.

For experimental purposes, cells were detached from flasks and plated in required density onto poly-D-lysine (Sigma-Aldrich Chemie, Schnelldorf, Germany) precoated coverslips ( $\varnothing 11 \mathrm{~mm}$, A.Hartenstein, Würzburg, Germany) placed into multiwell plates (Nunclon, 4 wells multidish, Thermo Fisher Scientific). Cells were allowed to adhere to the coverslips for $1 \mathrm{~h}$ at RT after which Grace's /10\% FBSG medium was added. Cell cultures were maintained in the incubator at $20^{\circ} \mathrm{C}$ for at least $24 \mathrm{~h}$ before any experimental treatment to allow enough time for cells to settle down and flatten. 
III.2.1.2 Experimental protocol for studying the effect of Epo on proliferation and differentiation in the Manduca sexta cell line

For proliferation experiments, approximately 15000 cells per coverslip were plated and cultured in $500 \mu \mathrm{l}$ of Grace's/10\% FBSG medium. After $24 \mathrm{~h}$ the medium was removed and serum-free Grace's medium with dissolved EdU (1 mM) (Molecular Probes, Thermo Fisher Scientific) was added. For treatment of cultures with Epo, the medium was additionally supplemented with rhEpo (4 $\mathrm{U} / \mathrm{ml}$ ). Cells were subsequently cultured for 2 days at $20^{\circ} \mathrm{C}$. Afterwards cells were fixed and processed to visualize EdU labeling.

For experiments in which the influence of Epo on cell differentiation was studied, approximately 30000 cells per coverslip were plated and cultured in $500 \mu \mathrm{l}$ of Grace's/10\% FBSG medium. After $24 \mathrm{~h}$ the culture medium was removed and replaced with either serum-free medium, serum-free medium containing rhEpo $(4 \mathrm{U} / \mathrm{ml})$ or full medium (Grace's/10\% FBSG). Cells were cultured in these media for 4 days at $20^{\circ} \mathrm{C}$ and afterwards fixed and stained for anti-HRP immunofluorescence.

\section{III.2.1.3 Detection of incorporated EdU via "click“ reaction}

Cell proliferation was assessed by EdU incorporation. EdU (5-ethynyl-2'deoxyuridine) is a nucleoside analog of thymidine that is readily incorporated into newly synthesized DNA during replication and can be detected using a "click" chemical reaction. The click reaction involves a copper (I)-catalyzed formation of a stable triazole ring from a terminal alkyne group of EdU and an azide moiety of a modified fluorescent dye. The main advantage of the EdUbased assay over the alternative BrdU assay is that fluorescent azides due to their small size have better access to terminal alkyne groups of incorporated EdU molecules compared to larger-sized antibodies that are used to detect incorporated BrdU. In addition, the EdU detection method is compatible with immunostaining (Salic \& Mitchison 2008). 
To detect EdU staining, after fixation with $4 \%$ paraformaldehyde in $0.1 \mathrm{M}$ phosphate buffer for 30 minutes at RT and 3 washes for 5 minutes in $0.1 \mathrm{M}$ phosphate buffer, followed by 3 washes for 5 minutes in tris-buffed saline (TBS), cell-containing coverslips were incubated with staining solution for 30 min at RT. Staining solution was freshly made each time and consisted of 100 $\mathrm{mM}$ tris (pH 8.5) (Sigma-Aldrich), $1 \mathrm{mM} \mathrm{CuSO} 4$ (Sigma-Aldrich), $10 \mu \mathrm{M}$ tetrametyl rhodamine (TAMRA) azide (Molecular Probes, Thermo Fisher Scientific) and $100 \mathrm{mM}$ sodium ascorbate (Sigma-Aldrich), which was added last to the mix (immediately before addition to the cells). After staining, the cells on coverslips were washed 3 times with TBS for $5 \mathrm{~min}$. After 3 washes for $5 \mathrm{~min}$ in PBS, cells were incubated with DAPI dissolved in PBS for $30 \mathrm{~min}$ at RT. Excess of dye was removed by 3 washes with PBS for $5 \mathrm{~min}$. Coverslips were mounted in DABCO (Sigma-Aldrich) and stored at $4{ }^{\circ} \mathrm{C}$ in the dark until analysis with confocal microscopy.

III.2.1.4 Anti-HRP, anti-RePo, anti-synapsin and anti-fasciclin II immunochemistry

Cells were fixed with $4 \%$ paraformaldehyde in $0.1 \mathrm{M}$ phosphate buffer for 30 minutes at RT and rinsed 3 times for 5 minutes with $0.1 \mathrm{M}$ phosphate buffer. To facilitate antibody penetration, cells were permeabilized by 5 washes in PBS containing $0.1 \%$ Triton-X-100 (0.1\% PBS-T) for 5 minutes each, at RT. Cells were then incubated for 30 min with blocking solution before addition of primary antibodies. Blocking solution consisted of $0.25 \%$ bovine serum albumin (BSA) (MP Biomedicals, Heidelberg, Germany) and 5\% normal goat serum (NGS, Jackson Immuno Research Laboratories, West Grove, Pennsylvania, USA) in $0.1 \%$ PBS-T. After blocking, cells were incubated overnight at $4^{\circ} \mathrm{C}$ with primary antibodies diluted in blocking solution. The following antibodies were used: anti-HRP from rabbit (1:500 dilution, Sigma-Aldrich), anti-RePo from mouse (1:50 dilution, 8D12, Developmental Studies Hybridoma Bank, DSHB, Iowa, USA), anti-synapsin from mouse (1:100 dilution, nc46, DSHB) and anti-fasciclin II from mouse (1:50 dilution, 2F5, DSHB). After 5 washes for 5 min each in $0.1 \%$ 
PBS-T cells were incubated with corresponding secondary antibodies diluted in $0.1 \%$ PBS-T for 1 h at RT. For anti-HRP detection a Cy2-coupled anti-rabbit antibody from goat (Rockland Immunochemicals, Limerick, Pennsylvania, USA) was used, Anti-RePo, anti-synapsin and anti-fasciclin II detection was achieved with an AF488-coupled anti-mouse antibody from goat (Invitrogen, Molecular Probes, Thermo Fisher Scientific). When needed, cells were incubated with DAPI (1:1000) together with the secondary antibody. After 5 washes for $5 \mathrm{~min}$ in $0.1 \%$ PBS-T and 3 washes for $5 \mathrm{~min}$ in PBS, cells were mounted in DABCO. The preparations were stored in folders protected from light and moisture at $4^{\circ} \mathrm{C}$ until analysis.

The protocol applied for double anti-HRP and anti-synapsin immunolabeling was similar to single immunostaining protocols, with the only difference related to the secondary antibodies that were applied. To detect anti-HRP a Cy2-coupled anti-rabbit antibody from goat (Rockland) was used, while for the detection of anti-synapsin an AF633 anti-mouse antibody from goat (Invitrogen, Molecular Probes, Thermo Fisher Scientific) was used.

\section{III.2.1.5 Analysis of proliferation and differentiation}

Analysis of proliferation rates and differentiation rates was performed with a confocal microscope (Leica TCS-SP8, Leica Microsystems, Wetzlar, Germany). For proliferation experiments, 36 non-overlapping fields from each coverslip were scanned with a 20x objective using two channels. In the first channel, a 405-nm line from a diode laser was used to excite DAPI and emission was detected from 420 to $470 \mathrm{~nm}$. In the second channel, EdU/TAMRA fluorescence was excited with the 561-nm line of a HeNe laser and emitted fluorescence collected from 570 to $680 \mathrm{~nm}$. For differentiation assays, 10 fields from each coverslip were scanned with a 20x objective using two channels. While for the DAPI emission and detection the same settings were used as described above, in the second channel fluorescence of Cy2 bound to anti-HRP was excited with the 488-nm line of an argon laser and the resulting emission detected from 500 
to $620 \mathrm{~nm}$. Viable cells were identified based on their DAPI staining pattern and total numbers of viable DAPI-stained nuclei and EdU-positive nuclei were counted from each image. The results are expressed as the percentage of EdUpositive nuclei of the total number of DAPI stained nuclei normalized to control values. Similarly, the rate of differentiation was quantified by counting the total number of both, viable anti-HRP-positive and viable DAPI-stained cells and determination of the percentage of anti-HRP-positive cells from the total number of counted cells per experimental condition. A „cell counter" plugin of ImageJ software (National Institute of Health, Bethesda, USA) was used to count cells of different categories. Results are displayed in box graphs generated using the OriginPro (version 8.5, OriginLab Corporation) software package. The differences in data population distributions (averages of EdUpositive cells and anti-HRP-positive cells between the control and Epo-treated groups) were analyzed using nonparametric Kruskal-Wallis tests followed by Mann-Whitney $U$ test. $P$ values smaller than 0.05 were considered as significantly different.

Anti-RePo, anti-synapsin and anti-fasciclin II fluorescence was only qualitatively analyzed using the confocal microscope TSC SP8.

\section{III.2.2 Primary cultures of locust optic lobe cells}

\section{III.2.2.1 Preparation of locust optic lobe primary cultures}

Primary cultures of locust optic lobe cells were established from fourth-instar nymphs of Locusta migratoria purchased from a commercial breeder (Hintze, Berlin, Germany) and maintained in colonies at $22-25^{\circ} \mathrm{C}$ on a $12 / 12 \mathrm{~h}$ day/night cycle. In brief, optic lobes (OL) were dissected from the brains of Locusta migratoria and collected in culture dishes filled with Leibovitz`s L-15 medium (L15, Gibco, Life Technologies, Thermo Fisher Scientific) supplemented with $0.5 \%$ gentamicin (GM, BioReagent, Sigma-Aldrich). After cleaning in L15/0.5\%GM, OL were incubated for 15 min with a mixture of 
collagenase and dispase (1 mg/ml, Sigma-Aldrich) dissolved in L15/0.5\%GM in an incubator (model B6120, Heraeus Instruments, Hanau, Germany) at $27^{\circ} \mathrm{C}$. After three short washings with Hanks`Balanced Salt Solution (HBSS, Gibco, Life Technologies), the tissue was mechanically dissociated by gentle trituration and spinned down with a bench rotor for $1 \mathrm{~min}$. The pellet of dispersed optic lobe cells was resuspended in L15/0.5\%GM. Dissociated cells were equally distributed onto ConA-coated coverslips. After $2 \mathrm{~h}$ the culture dishes were filled with L15/0.5\%GM supplemented with 5\% FBSG (2ml/culture dish) and placed in an incubator at $27^{\circ} \mathrm{C}$ in a humidified atmosphere.

\section{III.2.2.2 Experimental protocol for studying the effect of Epo on locust optic lobe cell proliferation and differentiation}

After $24 \mathrm{~h}$ the medium was replaced with serum-free L15/0.15\%GM and cell cultures returned back to the incubator at $27^{\circ} \mathrm{C}$ for two hours. Subsequently, the medium was replaced with L15/0.15\%GM supplemented with 1mM EdU and with rhEpo (1 U/ml and $10 \mathrm{U} / \mathrm{ml}$, Epo-treated groups) or without rhEpo (control groups). Cell cultures exposed to these media were further cultured for 2 days at $27^{\circ} \mathrm{C}$.

\section{III.2.2.3 EdU staining and anti-HRP immunocytochemistry}

After 2 days of culturing under above described conditions, Epo-treated and control cell cultures were fixed and processed for detection of incorporated EdU and immunodetection of HRP. Fixation was performed with $4 \%$ paraformaldehyde in $0.1 \mathrm{M}$ phosphate buffer for $30 \mathrm{~min}$ at RT. After 3 washes in phosphate buffer ( 5 min each), followed by 2 washes in PBS and 3 washes in $0.1 \%$ PBS-T, cells were incubated with blocking solution $(0.25 \%$ BSA $/ 5 \%$ NGS/0.1\% PBS-T) for $30 \mathrm{~min}$ at RT. The primary antibody anti-HRP from rabbit (Sigma-Aldrich) diluted (1:500) in blocking buffer was applied overnight at $4^{\circ} \mathrm{C}$. Excess antibody was removed by 3 washes in 0.1\% PBS-T and 2 washes in PBS. Cell cultures were afterwards prepared for EdU-staining by 3 washes in TBS. 
Staining solution (100 mM Tris, pH 8.5; 1 mM CuSO $; 10 \mu$ TAMRA-azide; 100 $\mathrm{mM}$ sodium ascorbate) was applied for $30 \mathrm{~min}$ at RT. After 3 washes with TBS, cells were washed 3 times with $0.1 \%$ PBS-T and incubated with secondary antibody AF488-coupled anti-rabbit antibody from goat (Invitrogen, Molecular Probes, Thermo Fisher Scientific) diluted (1:300) in $0.1 \%$ PBS-T for $1 \mathrm{~h}$ at RT. DAPI was diluted (1:1000) together with the secondary antibody and applied simultaneously. After 5 washes with $0.1 \%$ PBS-T, cells were finally mounted in DABCO for microscopic analysis. Until analysis, microscopic slides were kept at $4^{\circ} \mathrm{C}$ in the dark.

\section{III.2.2.4 Data analysis}

To analyze the staining, on average 60 regions of each coverslip were scanned in systematic order covering one line between two opposite edges of the coverslip using confocal microscopy (Leica TCS-SP8). A 63x objective was used. DAPI and EdU staining were scanned in one sequence. A 405-nm line from a diode laser was used to excite DAPI and emission was detected from 420 to 470 nm, while for EdU a 561-nm line of HeNe laser was used for excitation and emitted fluorescence was collected from 570 to $680 \mathrm{~nm}$. Fluorescence coming from anti-HRP was scanned in the next sequence using a 488-nm line of argon laser to excite dye and range of spectra from 500 to $550 \mathrm{~nm}$ to detect emitted signals. Only viable cells (as determined by the pattern of DAPI staining; median of surviving cells was $49.04 \%$ in control group; $52.14 \%$ in $1 \mathrm{U} / \mathrm{ml}$ Epotreated group; $50.57 \%$ in $10 \mathrm{U} / \mathrm{ml}$ Epo-treated group) were included in the analysis. Total numbers of EdU-positive cells, anti-HRP-positive cells, double anti-HRP/EdU-positive cells and anti-HRP/EdU-negative cells are counted with the assistance of the "cell counter" plugin of ImageJ software. The portions of EdU-positive and anti-HRP-positive cells were expressed as percentages of the total number of viable cells (set to 100\%). Results are displayed in box-plot graphs generated with the OriginPro software package. Due to their sparse occurrence the category of double anti-HRP/EdU-positive cells was not 
included in the graphical presentation of results. The differences in data population distributions (averages of EdU-positive cells and anti-HRP-positive cells between the control and Epo-treated groups) were analyzed using the nonparametric Kruskal-Wallis test followed by a Mann-Whitney $U$ test. $P$ values smaller than 0.05 were considered as significantly different.

\section{III.2.3 In vivo studies with Tribolium castaneum}

\section{III.2.3.1 Animals}

Red flour beetles Tribolium castaneum (wild type strain San Bernardino) were reared and maintained in plastic boxes $(15 \mathrm{~cm} \times 15 \mathrm{~cm} \times 10 \mathrm{~cm})$ filled with $1-2$ $\mathrm{cm}$ of whole wheat flour supplemented with $5 \%$ of dried yeast powder in climate chambers at $26^{\circ} \mathrm{C}$ (and approx. $40 \%$ humidity) on a 12/12-hours day/night cycle.

\section{III.2.3.2 EdU injection}

Freshly eclosed female adults (developmental stage identified based on lightly tanned cuticle and sex identified by size of genital papillae which are much larger in females than in males) were cooled down and fixed ventral side up on modeling clay. Using a microinjector (FemtoJet express, Eppendorf, WesselingBerzdorf, Germany) animals were injected via pulled glass capillary (borosilicate capillary with inner filament, 1.0/0.57 mm OD/ID, KBF-110075; Flaming/brown micropipette puller: model P-97, Sutter Instrument, Novato, CA, USA) into the lateral abdomen between third and fourth segment. Each animal was injected with approximately $0.5 \mu \mathrm{l}$ of injection solution, which was first pipetted as a drop onto an upside down Eppendorf cup and then brought into the glass capillary through the tip by immersing it into the drop (passively by capillary forces). The injection solution consisted of $10 \mathrm{mM} E d U$ diluted in Tribolium injection buffer $\left(1.4 \mathrm{mM} \mathrm{NaCl}, 0.007 \mathrm{mM} \mathrm{Na} \mathrm{HPO}_{4}, 0.03 \mathrm{mM}\right.$ $\mathrm{KH}_{2} \mathrm{PO}_{4}, 4 \mathrm{mM} \mathrm{KCl}$ ) supplemented with $10 \mathrm{U} / \mathrm{ml}$ rhEpo (Epo-treated group) or 
with equivalent volume of Epo solvent (control group). After injection animals were placed into small tubes containing flour with $5 \%$ yeast powder at RT for $24 \mathrm{~h}$ until brains were dissected.

\section{III.2.3.3 EdU- and immuno- staining of whole mount brains}

Brains of EdU-injected control and Epo-treated animals were dissected out $24 \mathrm{~h}$ after injection. After chilling on ice, animals were pinned ventral side up to the surface of a sylgard-coated plate with insect pins through their abdomen and brains were dissected after removing mouth parts and joined pieces of head cuticle. Dissections were performed in PBS. Since the hard cuticle was firmly attached to the head muscles, complete removal of all pieces of cuticle at this stage usually led to disruption of the brain tissue. To preserve tissue integrity brains were exposed by opening of the head capsule, dissected in connection with firmly attached cuticle and fixed with $4 \%$ paraformaldehyde in $0.1 \mathrm{M}$ phosphate buffer for $1 \mathrm{~h}$ at RT. After 3 washes in phosphate buffer (10 $\mathrm{min}$ each), the tissue was incubated with 10\% PBS-T (pH 7.4) for 1 to 2 days at $4^{\circ} \mathrm{C}$ to induce softening of the cuticle and permeabilization of underlying tissue. Remnants of the head capsule were afterwards removed using forceps leaving clean brains. Brains were incubated for $2 \mathrm{~h}$ at RT with blocking solution containing 5\% NGS and 0.25\% BSA dissolved in PBS with $0.3 \%$ Triton-X-100 (0.3\% PBS-T). Primary antibody anti-DCO from mouse (dilution 1:1000, provided by Prof. Dr. Daniel Kalderon, Columbia University, New York, USA) diluted in blocking solution was incubated with brain samples over night at $4^{\circ} \mathrm{C}$. Anti-DCO is a polyclonal antibody against the catalytic subunit of Drosophila melanogaster protein kinase A. This antibody has been shown to have a high affinity for mushroom body intrinsic neurons in a range of insect species (Skoulakis et al. 1993; Farris 2005) including Tribolium castaneum (Zhao et al. 2008). After 2 h washing with $0.3 \%$ PBS-T and 30 min washing with TBS, brains were transferred into the staining solution for EdU-detection (100 mM tris, $\mathrm{pH}$ 8.5; $1 \mathrm{mM} \mathrm{CuSO}$; $10 \mu \mathrm{M}$ TAMRA-azide; $100 \mathrm{mM}$ sodium ascorbate) for $1 \mathrm{~h}$. 
After 30 min washing with TBS and 30 min washing with $0.3 \%$ PBS-T, brains were incubated with secondary antibody, Cy5 goat anti-mouse (dilution 1:300, Jackson Immuno Research), AF488-coupled anti-HRP from Goat (dilution 1:300, Jackson Immuno Research) and DAPI (1:1000) diluted in 0.3\% PBS-T for $2 \mathrm{~h}$ at RT. Brains were afterwards washed in $0.3 \%$ PBS-T for $30 \mathrm{~min}$ at RT and mounted in DABCO onto slides containing spacers, followed by coverslipping.

\section{III.2.3.4 Confocal microscopy and image processing}

Stained whole mounts were analyzed with confocal microscopy (Leica TCSSP8) with 20x or $63 x$ glycerol immersion objectives. The lasers used to excite fluorescence and detection parameters were selected according to the absorption and emission properties of the applied dyes. Fluorescence coming from anti-DCO immunostaining was used to identify the mushroom bodies within whole mount preparations. Once mushroom bodies have been identified, they were scanned using a $63 x$ objective with a $1 \mu \mathrm{m}$ distance between optical sections along the z-axis. Single optical sections or projections made from a small number of optical sections were selected from stacks of each stained brain. These images were then adjusted for brightness and contrast using ImageJ software. 


\section{III.3 Results}

\section{III.3.1 Characterization of Manduca sexta cell line}

As not much is known about this cell line, a brief characterization was performed. Morphological analysis of the cell line using light microscopy with phase contrast revealed that most of the cells have a spindle-shaped form, with long bipolar processes, rather than branched extensions (Fig.28A, left). At least a subset of the cells were mitotically active, as demonstrated by detection of the nuclear thymidine analog EdU that was incorporated during DNA replication in the S-phase of the cell division (Fig. 28A, middle). Because most of the cultured cells displayed neuron-like morphology the cells were next stained for the insect neuronal marker anti-HRP. Anti-HRP antibodies specifically detect glycoproteins on the cell membrane and in membrane vesicles of insect neurons (Jan \& Jan 1982; Sun \& Salvaterra 1995) including cultured moth neurons (Lucas \& Nagnan-Le Meillour 1997; Tucker \& Tolbert 2003). Interestingly, immunocytochemical analysis revealed very few anti-HRP-positive cells (Fig. 28B, right). Identified neurons displayed extensions whose patterns seemed to differ from the majority of the cells observed with light microscopy. There were anti-HRP-positive cells with one, two or more extensions and with branches of variable complexity. Moreover, among the anti-HRP-positive cells some cells displayed only weak anti-HRP immunofluorescence and had only few antiHRP positive vesicles, whereas other cells displayed the typical anti-HRP phenotype known from stainings in other insects (Fig. 26).

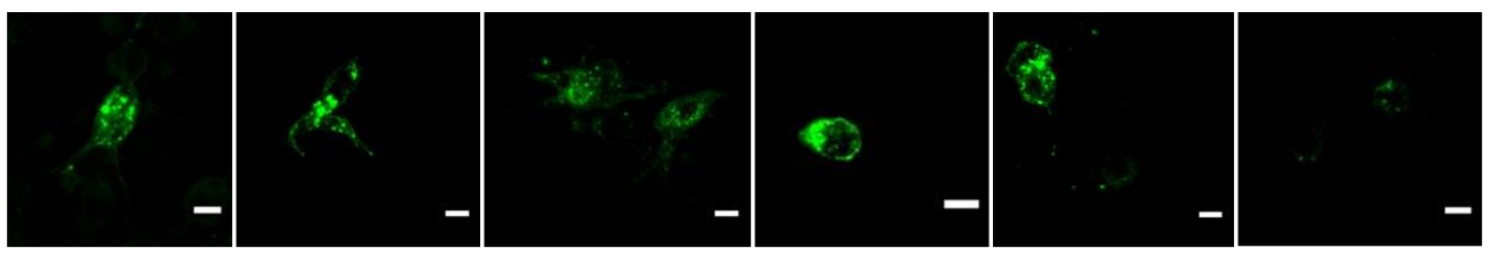

Figure 26. Anti-HRP immunostaining of the Manduca sexta cell line. Anti-HRP-positive cells (green) display different patterns of cellular processes. Some anti-HRP-positive cells are only weakly immunostained, characterized by low number of anti-HRP-positive vesicles and absence of processes (last two images, right). Scale bars: $10 \mu \mathrm{m}$. 
On the other hand, almost all cells were positive for anti-synapsin antibodies (Fig. 27, upper panel, left). These antibodies specifically detect the synapseassociated protein of 47kDa (SAP47) expressed in both larval (Saumweber et al. 2011) and adult insect brains (Reichmuth et al. 1995). The presence of few antisynapsin-negative cells in the culture cannot be completely excluded, as this has not been systematically investigated. Double immunostaining with antibodies against HRP and SAP47 (anti-synapsin) revealed that all detected anti-HRPpositive cells of the $M$. sexta cell line cultures were also anti-synapsin positive (Fig. 27, upper panel, right). Furthermore, a large portion, but not all cells within the cell line were positive to anti-fasciclin II antibodies (Fig. 27, lower panel). Fasciclin II is a homophilic cell adhesion molecule of the immunoglobulin superfamily that is involved in the fasciculation and guidance of axons in developing insect nervous systems (Bastiani et al. 1987; Harrelson \& Goodman 1988; Grenningloh et al. 1991). Again, all detected anti-HRP-positive cells were also anti-fasciclin II positive (Fig. 27, lower panel, right).

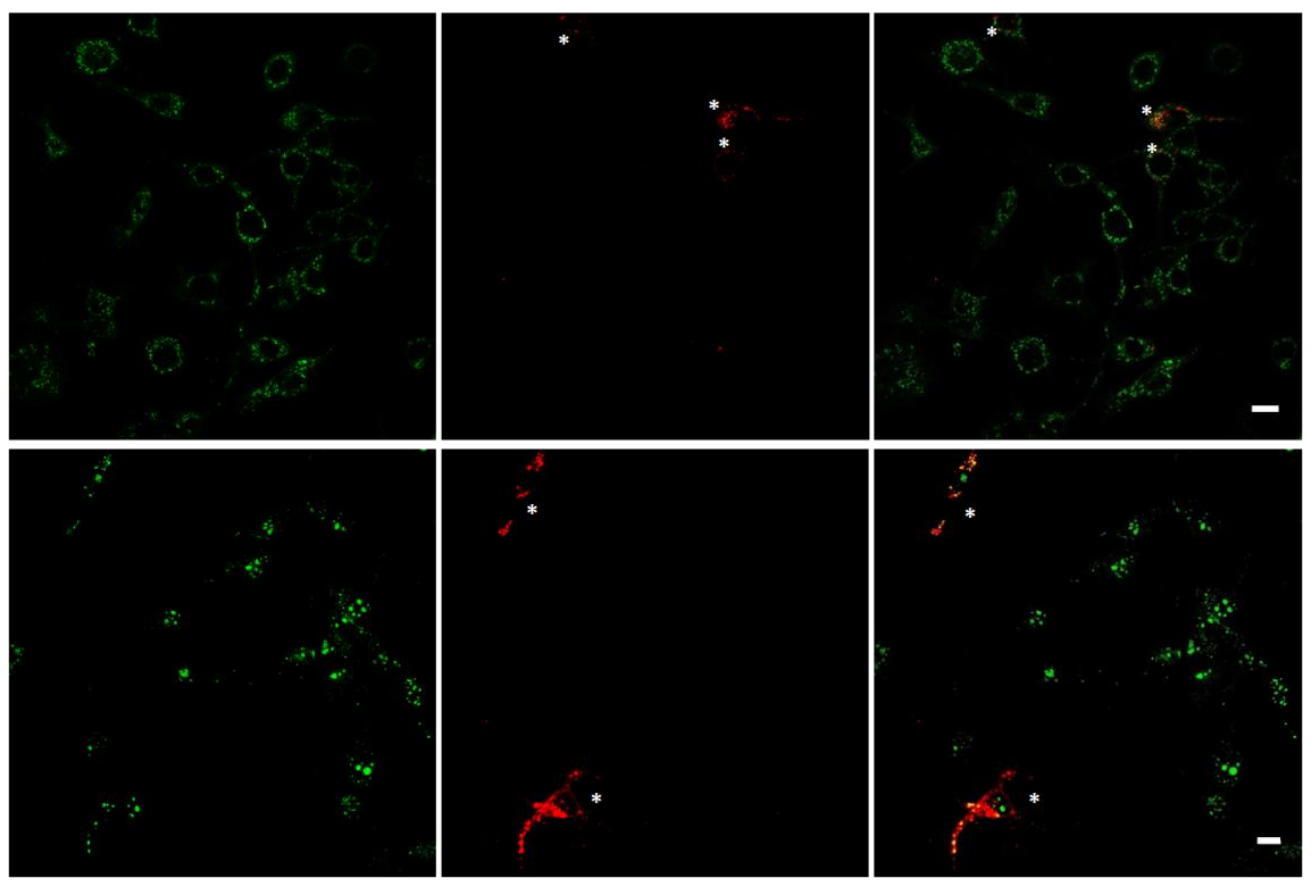

Figure 27. Anti-synapsin (upper panel, green) and anti-fasciclin II (lower panel, green) immunostaining of the Manduca sexta cell line in conjunction with anti-HRP immunostaining (upper and lower panel, red). Upper panel: while most cells in the cell line are positive for antisynapsin staining indicating their neuron fate commitment, only few cells are positive for the pan-neuronal anti-HRP marker $\left(^{*}\right)$. Lower panel: only two anti-fasciclin II-positive cells are also positive for anti-HRP $\left.{ }^{*}\right)$. Right: overlay of two stainings. Scale bars: $10 \mu \mathrm{m}$. 
Additionally, all cultured cells were negative for the glial marker anti-RePo (images not shown). Anti-synapsin and anti-fasciclin II staining indicated that the cell line consisted largely of cells with neuronal identities of which at least a subset had the capacity to proliferate, as indicated by EdU staining. At least some of the daughter cells had the ability to undergo progressive maturation that resulted in fully developed neuronal anti-HRP phenotype. Hence, EdUstaining was chosen to label proliferation, while anti-HRP immunoreactivity was chosen to analyze neuronal differentiation in subsequent experiments.

To determine the amount of cells displaying signs of mature differentiated neurons that express HRP, a preliminary experiment with different durations of culturing after plating, namely 2, 4, 6 and 8 days, was performed. After fixation and subsequent immunostaining against HRP, the portion of immunopositive neurons was determined. The percentage of anti-HRP-positive cells after 2 days of growing under standard conditions in the absence of serum was $1.01 \%$; after 4 days $1.76 \%$; after 6 days $2.11 \%$ and after 8 days $1.39 \%$. Although the portion of anti-HRP-positive cells slightly increased from day 2 until day 6 after plating, the difference was minimal, indicating that the duration of cell culture maintenance is not an important factor for neuronal differentiation. Since the total number of cells in the cultures decreased after 6 days of culturing (5922 cells after 2 days, 5682 cells after 4 days, 2687 cells after 6 days and 575 after 8 days), all subsequent experiments were performed within 4 days after plating of the cells.

\section{III.3.2 Epo does not stimulate Manduca sexta progenitor cell proliferation and their differentiation into neurons expressing the neuronal marker anti-HRP in vitro}

It has been shown that Epo can modulate different stages of vertebrate neurogenesis and therefore plays an important role during the proliferation and differentiation of vertebrate neural stem cells (Shingo et al. 2001; Wang et al. 
2004; Hassouna et al. 2016). Whether Epo also acts as a regulator of insect progenitor cell proliferation and differentiation has not previously been studied. To examine a potential influence of Epo on insect neural progenitor cell proliferation in vitro, the cells were exposed to rhEpo $(4 \mathrm{U} / \mathrm{ml})$ in the presence of EdU in serum-free medium for 48 hours. Control cultures were treated in the same way, but without exposure to Epo. In total 35061 cells from 5 experiments were analyzed. Results are displayed in Figure 4B (left) as percentages of EdUpositive nuclei from the total number of DAPI-stained nuclei which were normalized to $100 \%$. It is shown that Epo did not affect the amount of EdUlabeled nuclei, as the percentage of EdU-positive cells (median: 23.75\%) was not statistically different from control values (median: $25 \%, P=1$ ). These data suggest that Epo has no effect on the proliferation of Manduca sexta neural precursors in vitro.

To determine the effect of Epo on neural progenitor cell differentiation, the cells were treated with rhEpo for 4 days (Fig. 28B, right). After fixation cell cultures were immunostained for the pan-neuronal marker anti-HRP and the number of stained neurons was quantified. In total 86593 cells were analyzed from 6 experiments. The results are displayed as percentages of anti-HRP-positive cells relative to the total number of analyzed viable cells (set as 100\%). As shown in Fig. 28B (right) the percentage of anti-HRP-positive cells in Epo-treated cultures (median: $1.2 \%$ ) was not significantly different from the percentage of anti-HRPpositive cells in control cultures (median: $1.25 \% ; P=0.41)$. Serum itself $(10 \%$ FBSG) had either no effect on cell differentiation, as percentage of anti-HRPpositive cells in the cell cultures in medium supplemented with 10\% FBSG (median: 1.54\%; Fig.28B, right) did not significantly differ from the percentage of anti-HRP-positive cells in control cultures $(P=0.37)$. Thus, if the appearance of anti-HRP immunoreactivity can serve as a marker for neuronal differentiation, Epo has no stimulatory effect on differentiation of $M$. sexta neural progenitors into mature neurons in vitro. 
A

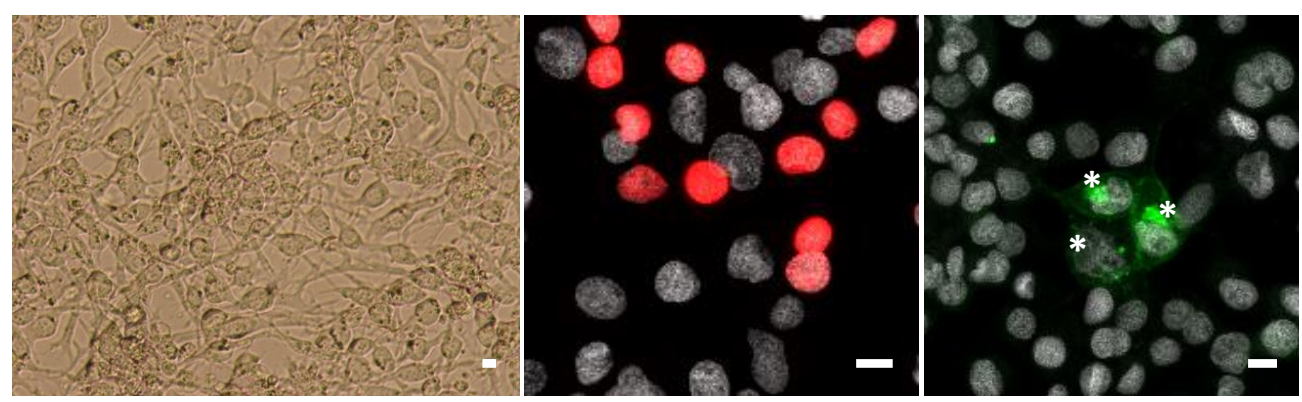

B
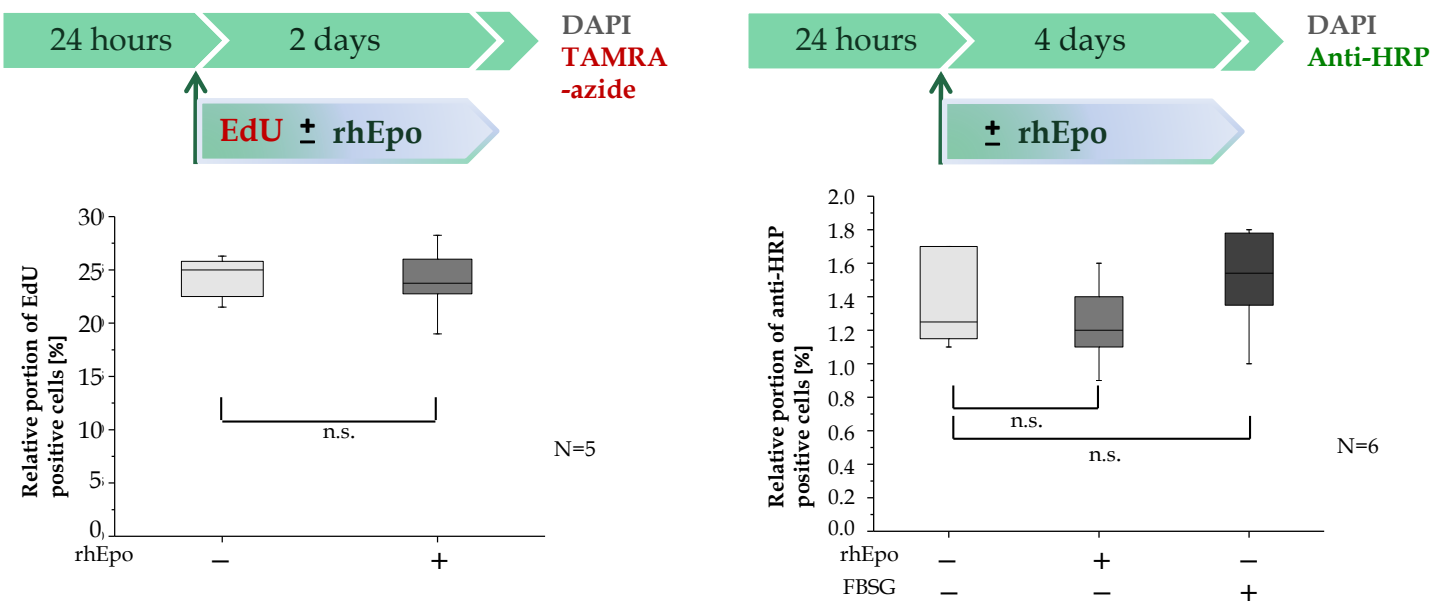

Figure 28. Assessment of the effects of Epo on proliferation and differentiation in the Manduca sexta cell line.

(A) Left: Phase-contrast image of the Manduca sexta cell line. Viable cells make anatomical contacts via long, mostly bipolar, processes extending from their cell bodies. Middle: Proliferation was analyzed by determining the portion of EdU-positive cells. Representative fluorescent micrography of a cell culture 2 days after treatment showing nuclei (with incorporated EdU) of recently proliferated cells (red). Nuclei of both, dividing and non-dividing cells are stained with DAPI (gray). Right: Differentiation rate was assessed by determining the portion of cells positive to anti-HRP. Fluorescent micrography of a cell culture 4 days after treatment stained with anti-HRP antibodies (green). Nuclei are stained with DAPI (gray). Three cells are positive for the pan-neuronal marker HRP (asterisks), while most cells are anti-HRPnegative. Scale bars: $10 \mu \mathrm{m}$.

(B) RhEpo (4 U/ml) does not influence proliferation or differentiation in the Manduca sexta cell line. Relative portion of EdU-positive cells does not differ between rhEpo-treated and untreated cells (left). Similarly, rhEpo did not change the portion of anti-HRP-positive cells (right). FBSG $(10 \%)$ has no effect on differentiation in the Manduca sexta cell line. Statistics: Kruskal-Wallis ANOVA with Mann-Whitney $U$-test. N.s. not significant. Schemes depicting the experimental procedures are illustrated above box plot charts. 


\section{III.3.3 Epo has no effect on proliferation and differen tiation of locust preadult optic lobe neuronal precursors in vitro}

A primary culture system established from optic lobes of locust nymphs was employed to investigate the effects of Epo on insect neurogenesis in vitro. After isolation, dissociation and plating, optic lobe cells were grown for $24 \mathrm{~h}$ under usual culturing conditions before the experimental treatments. To test whether Epo regulates optic lobe neural progenitor cell proliferation and differentiation cell cultures were incubated with serum-free medium containing EdU (control) or EdU and rhEpo (Epo-treated group) for 2 days $(48 \mathrm{~h})$ at $27^{\circ} \mathrm{C}$. After fixation cells were immunostained with anti-HRP antibodies, incorporated EdU detected with "click“ reaction and all nuclei stained with DAPI (Fig. 29B). Analysis revealed that a very small portion of OL cells underwent mitosis (around 2\% of the OL cells expressed the cell proliferation marker EdU) (Fig. $29 \mathrm{E}$ ), and over $80 \%$ of the OL cells expressed the insect pan-neuronal marker HRP (Fig. 29F) following 2 days of exposure to Epo. The proportion of EdUpositive cells in the experimental groups treated with Epo 1U/ml rhEpo $(2.23 \%)$ and $10 \mathrm{U} / \mathrm{ml}(3.5 \%)$ was found to be similar to that of the non-treated (control) group $\left(2.71 \%, P_{\text {Epo-treated }(1 \mathrm{U} / \mathrm{ml}) \text { vs. non-treated }]}=0.49, P_{[\text {Epo-treated }(10 \mathrm{U} / \mathrm{ml}) \text { vs. non-treated }]}=\right.$ 0.69) (Fig. 29C and 29E). Similarly, the proportion of anti-HRP-positive cells in cell cultures treated with $1 \mathrm{U} / \mathrm{ml}$ rhEpo (84.88\%) and $10 \mathrm{U} / \mathrm{ml} \mathrm{rhEpo}(81 \%)$ did not differ significantly from the proportion of anti-HRP-positive cells in nontreated cell cultures (control group) $\left(78.38 \% ; P_{[\text {Epo-treated }(1 \mathrm{U} / \mathrm{ml}) \text { vs. non-treated] }}=0.34\right.$; $\left.P_{[\text {Epo-treated }(10 \mathrm{U} / \mathrm{ml}) \text { vs. non-treated }]}=0.69\right)$. Additionally, a few EdU/anti-HRP doublepositive cells have been detected in both, Epo-treated and control cultures, revealing de novo generation of neurons from descendants of proliferating neural progenitors following their maturation and differentiation. However, their contribution to the total number of analyzed cells was extremely low (mean: $0.66 \%$ in the non-treated group, $1.2 \%$ in the group treated with $1 \mathrm{U} / \mathrm{ml}$ Epo, $0.45 \%$ in the group treated with $10 \mathrm{U} / \mathrm{ml}$ Epo; data not shown on graphs). Thus, Epo (1 and $10 \mathrm{U} / \mathrm{ml})$ did not influence OL cell proliferation and differentiation under conditions applied in my experiments. 
A

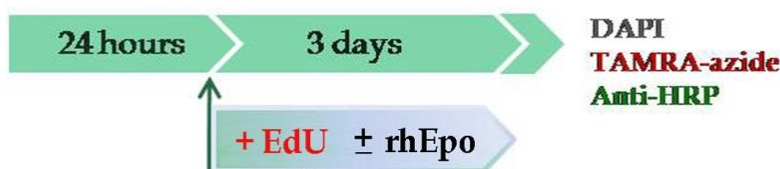

C
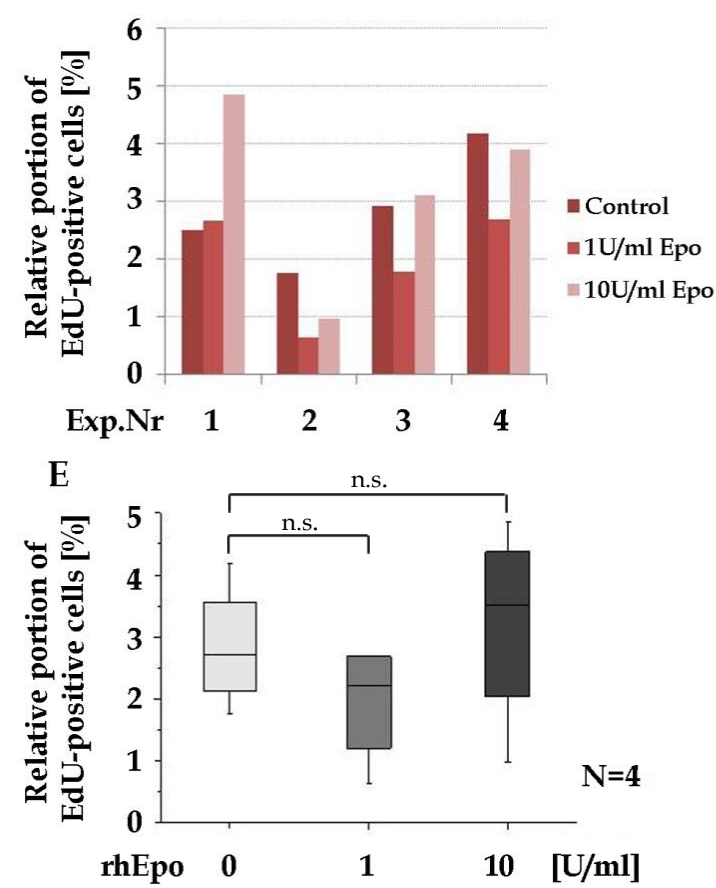

B

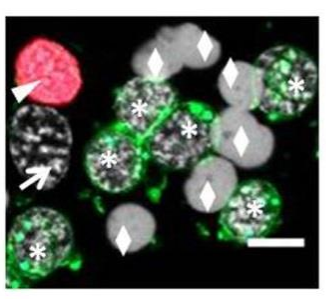

D

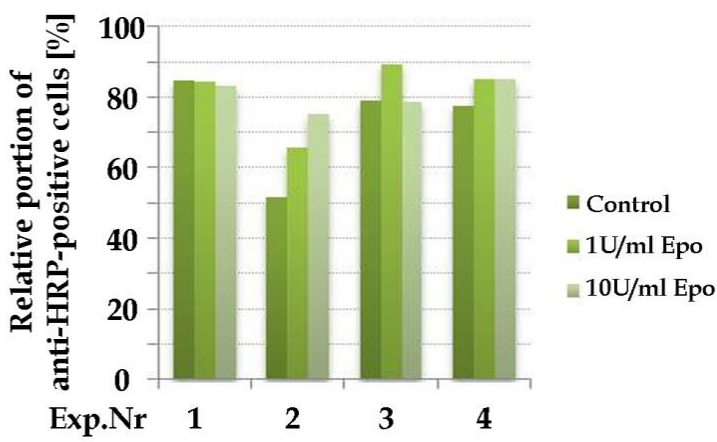

$\mathrm{F}$

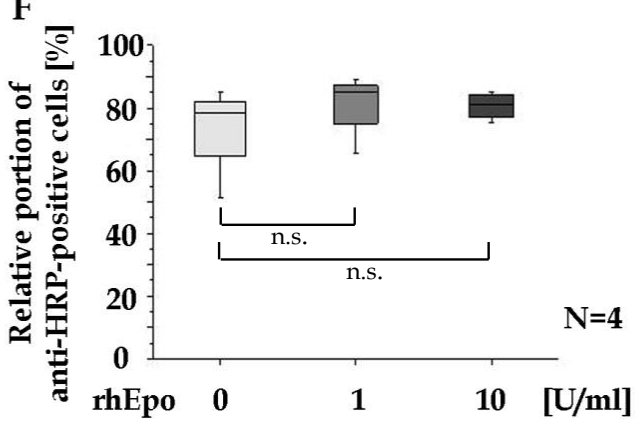

Figure 29. Epo does not stimulate proliferation and differentiation of primary cultured locust optic lobe cells. (A) Schematic drawing of experimental procedure. (B) Representative fluorescent micrography of a cell culture 2 days after treatment showing viable cells positive for the pan-neuronal marker HRP (asterisks), nuclei of viable anti-HRP-negative cells (arrow), nuclei of dead cells (rhomboids) and one nucleus of a viable, recently proliferated anti-HRPnegative cell (arrowhead). Anti-HRP (green), EdU/TAMRA (red), DAPI (gray). Scale bar: 10 $\mu \mathrm{m}$. Relative portion of EdU-positive cells after 3 days of incubation with $1 \mathrm{U} / \mathrm{ml}$ and $10 \mathrm{U} / \mathrm{ml}$ rhEpo of four individual experiments (C) and their medians (E). The proliferation rate was quantified as the ratio of EdU-positive cells to DAPI-labeled nuclei of viable cells normalized to $100 \%$. Relative portion of anti-HRP-positive cells after 3 days of incubation with $1 \mathrm{U} / \mathrm{ml}$ and 10 $\mathrm{U} / \mathrm{ml}$ rhEpo of four individual experiments (D) and their medians (F). Differentiation was quantified as the ratio of anti-HRP-positive cells to total number of viable cells normalized to 100\%. Statistics: Kruskal-Wallis ANOVA with Mann-Whitney U-test. N.s. not significant. 


\section{III.3.4 In vivo studies on the proliferation of Tribolium castaneum neuroblasts}

Since EdU/TAMRA staining specifically labeled nuclei of proliferating cells in two in vitro systems studied (see above), we applied the same technique to stain proliferating mushroom body neuroblasts in vivo in order to assess the potential influence of Epo on neurogenesis in this brain region. Analysis of confocal images of whole mount preparations of T. castaneum brains showed however that EdU staining cannot be unequivocally assigned only to proliferating nuclei. Figure 30 illustrates a region of the mushroom body neuropile identified by anti-DCO immunostaining (red) where dividing neuroblasts are expected to be located. The Anti-DCO antibody, which has a high affinity for specific proteins of mushroom body-intrinsic neurons, labels mature neurons, but not neuroblasts. Most Tribolium brains contain two mushroom body neuroblasts per hemisphere, although individual variations with some animals having one or three neuroblasts exist (Zhao et al. 2008). In Figure 30B and 30D four EdUlabeled nuclei (arrowheads) can be recognized. Additionally, numerous small round EdU/TAMRA-stained objects can be seen (arrows). However, from the staining pattern which does not display the clear chromatin structure revealed with DAPI staining (Fig. 30C and 30D) it is not possible to claim that they represent nuclei of dividing cells. Furthermore, in some whole mount preparations, EdU-positive cells within mushroom bodies could not be detected at all (figures not shown). Thus, EdU/TAMRA staining is not sufficiently reliable for proper identification and quantification of proliferating mushroom body neuroblasts in vivo. 


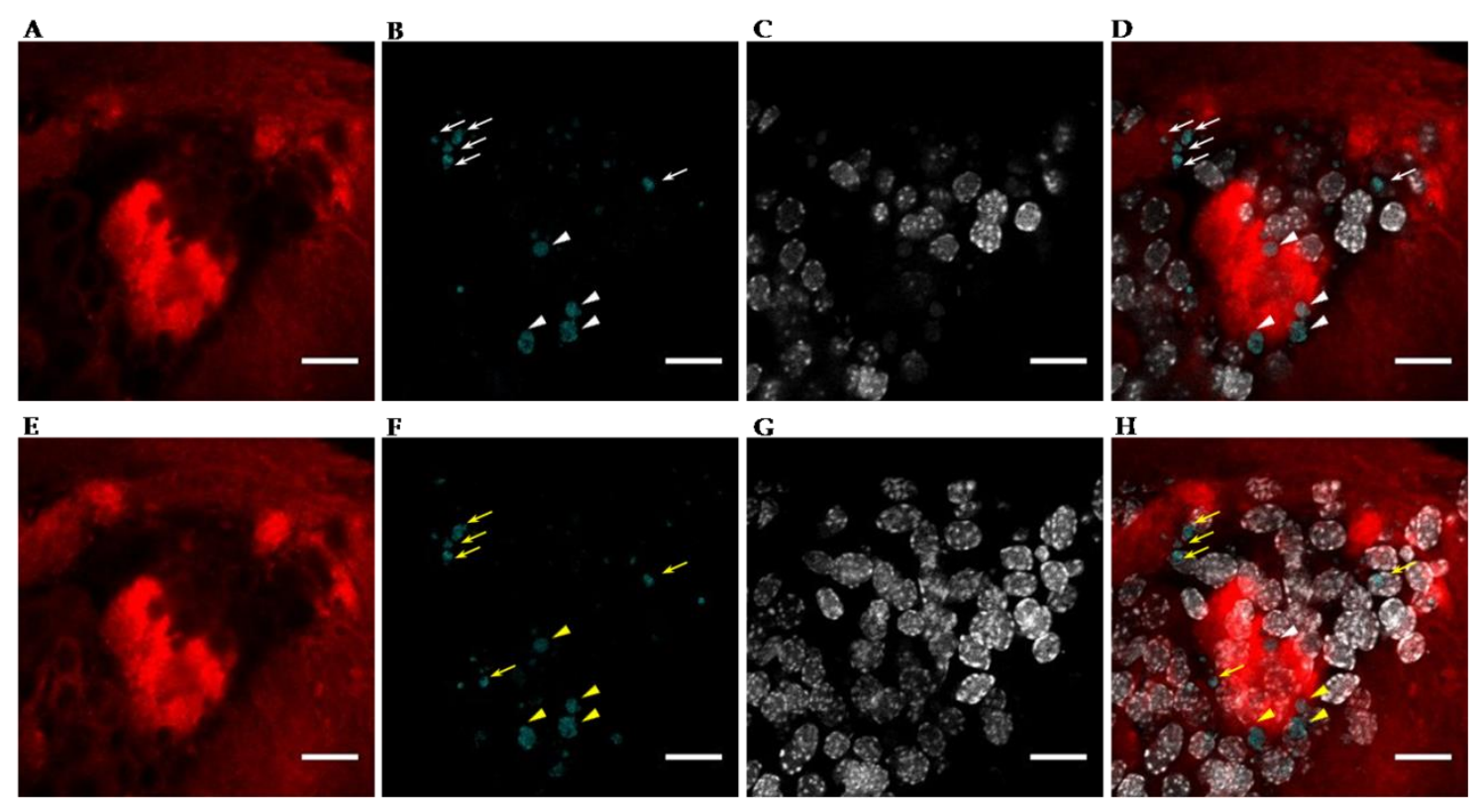

Figure 30. In vivo EdU staining in the brain of Tribolium castaneum. A-D: One optical section of the whole mount brain of a newly eclosed adult after $24 \mathrm{~h}$ of incubation with EdU and rhEpo. E$\mathrm{H}$ : Maximu m projections of four optical sections (all optical sections in which EdU staining was visible). (A, E) Anti-DCO immunostaining (red), (B, F) EdU staining (cyan), (C, G) DAPI staining (gray) and (D, I) overlay of three stainings. Progeny of dividing neuroblast have incorporated EdU (arrowheads), while other EdU-positive structures (arrows) cannot be unequivocally assigned to nuclei (B, D). Discontinuous DAPI staining reflects chromatin structure of nuclei (C). Images in the lower panel (E-H) show that nuclei of non-dividing cells can be reliably identified $(\mathbf{G}, \mathbf{H})$ based on DAPI staining, including the nuclei of the cells that are not clearly visible in one optical section and may thus appear as structures similar to those detected as false EdU-positive objects. In contrast, small-sized EdU-stained objects (yellow arrows) do not get lost in maximal z-projection of all optical sections in which they are detected. Even more of such staining emerges $(\mathbf{F}, \mathbf{H})$. Scale bars: $10 \mu \mathrm{m}$. 


\section{III.4 Discussion}

\section{III.4.1 Epo-mediated regulation of neurogenesis}

There is accumulating evidence for the involvement of Epo in the regulation of embryonic and adult neurogenesis in mammals. The ability of Epo to promote genesis of neuronal and oligodendroglial progenitors from NSC has been demonstrated both in vitro and in vivo (Shingo et al. 2001). Following ischemic brain injury Epo stimulates the production of neurons (Shingo et al. 2001; Wang et al. 2004) and oligodendrocytes (Kako et al. 2012; Jantzie et al. 2013) promoting the replacement of lost cells. The regulation of neurogenesis and gliogenesis affects diverse mechanisms that include proliferation of progenitor cells through repeated divisions, differentiation through selection and emergence of cell committed fate by some daughter cells and survival, which refers to the maintenance of newly generated cells and their functional incorporation into existing circuitry (Schoenfeld \& Gould 2012). While some studies reported that the neurogenic effect of Epo occurs primarily at the level of proliferation of neural progenitors (Ransome \& Turnley 2007; Chen et al. 2007b) other studies suggested that Epo selectively promotes neuronal differentiation (Shingo et al. 2001; Hassouna et al. 2016) or enhances both, proliferation of neural progenitors and their differentiation into neurons (Wang et al. 2004). Reports about the effects of Epo on oligodendroglial genesis following brain injury are more consistent. Epo has been found to promote survival and differentiation of oligodendrocyte precursor cells, without mediating a significant proliferative effect (Kako et al. 2012; Jantzie et al. 2013; Hassouna et al. 2016).

Whether Epo regulates insect neurogenesis has been examined using three different preparations: a permanent cell line, a primary culture and an in vivo preparation. I used insect species belonging to three different orders: Lepidoptera (Manduca sexta), Orthoptera (Locusta migratoria) and Coleoptera (Tribolium castaneum). The possible involvement of Epo in the regulation of neuroblast proliferation and differentiation into neurons at embryonic, late pupal and early adult developmental stages was studied. The obtained results 
however did not provide any evidence for a role of Epo in insect development and adult neurogenesis. Under the applied conditions, I could not demonstrate Epo-induced regulation of neurogenesis neither at the level of proliferation of progenitor cells, nor at the level of neuronal differentiation in any of the three preparations.

\section{III.4.2 Epo does not induce neurogenesis in a neural cell line derived from embryonic tissue of Manduca sexta}

The first model system I used to study the potential influence of Epo on embryonic neurogenesis in insects was the cell line established by Eide and colleagues. This cell line was established from primary cultures of embryonic tissue of $M$. sexta and has been described as a line consisting almost entirely of cells of a single type that have the appearance of fibroblasts (Eide et al. 1975). Few cells with epitheloid shape that were also observed in the line were claimed to be morphological variants of the same cell type (Eide et al. 1975). The previously described morphological features of the cell line were confirmed in our analysis using phase-contrast microscopy which revealed many spindleshaped cells with extended bipolar processes occasionally accompanied by cells having a discoid shape. Although anti-RePo antibody is widely used to label glia in Drosophila (Xiong et al. 1994), its specificity for glia in M. sexta species has not yet been demonstrated and therefore the absence of anti-RePo immunoreactivity does not necessarily imply the absence of glial cells in this cell line. Nonetheless, qualitative immunocytochemical analysis of antisynapsin, anti-fasciclin II and anti-HRP staining suggested that the cell line consists of cells with neuronal identities, with several subpopulations possibly representing different developmental stages of neuronal lineage cells. A certain pool of cells within the line seems to be responsible for the self-renewal of the neural progenitors, as demonstrated via EdU incorporation and detection. However, the cells seem to have weak capacity to reach the stage of mature neurons that express the insect pan-neuronal marker HRP. In all experimental 
paradigms applied not more than $2 \%$ of HRP-immunoreactive neurons were detectable in cell cultures. In fact, it has been shown that during postembryonic neurogenesis in $M$. sexta, the progeny of neuroblasts appear to arrest their differentiation soon after they elaborate axon-like processes into the neuropil. The cells remain arrested in this postmitotic, immature stage until the initiation of metamorphosis at the onset of the wandering stage during which the cells enter the phase of terminal differentiation and assume the morphology of mature functional neurons (Booker \& Truman 1987). Cellular factors stimulating terminal differentiation of neurons in $M$. sexta are not known and they might have been absent in standard culturing conditions of the M. sexta cell line. It is known that normal culturing conditions for cell lines, e.g. human SH-SY5Y cells, support continuous proliferation and growth of undifferentiated cells that have a neuroblast-like morphology and express markers for immature but not mature neurons. Addition of specific factors that stimulate differentiation causes a change in the properties of the cell line that starts to exhibit a decrease in proliferation rate and increase in expression of neuronspecific markers (Gordon et al. 2013). Anti-HRP labels a neuron-specific membrane glycoprotein coded by the nervana gene in Drosophila. However, antiHRP antibodies recognize a carbohydrate epitope, rather than protein. Although Nervana protein is synthesized very early in neurogenesis, glycosylation with the anti-HRP epitope occurs in later stages of neuronal maturation. As a consequence, while anti-Nervana immunostaining can be detected during the early stages of neurogenesis, anti-HRP staining is not observed until later stages of development (Sun \& Salvaterra 1995). Drosophila nac (neurally altered carbohydrate) mutant, which has a defect in the glycosylation pathway, does not show typical anti-HRP nervous system staining in adults, although anti-Nervana immunoreactivity can be detected in all developmental stages (Katz et al. 1988; Sun \& Salvaterra 1995). Therefore, low levels of anti-HRP immuno-detection in the $M$. sexta cell line can be also due to a decreased glycosylation of Nervana protein. 
As the presence of neural progenitors capable of dividing has been demonstrated by an EdU-based assay, the question whether Epo can stimulate their proliferation could be studied. The exposure of cell cultures to rhEpo for 48 hours had no significant effect on the proliferation of $M$. sexta neural progenitors compared to the proliferation of non-treated cell cultures. Thus, the data indicate that rhEpo does not regulate neurogenesis of the $M$. sexta progenitors in vitro at the level of proliferation.

The specificity of the anti-HRP antibody in detection of insect neurons has been confirmed in numerous studies using a wide range of insect species, including moths (Lucas \& Nagnan-Le Meillour 1997; Tucker \& Tolbert 2003). Since antiHRP-positive cells in the M. sexta cell line displayed a staining pattern characteristical for other anti-HRP-positive neurons, with intensively stained plasma membrane, neurites and vesicles, anti-HRP immunoreactivity was used as a marker for neuronal differentiation. To quantify the potential effect of Epo on the differentiation of $M$. sexta progenitors into neurons, the percentage of anti-HRP-positive cells in the cell cultures exposed to rhEpo for 4 days was compared with the percentage of anti-HRP-positive cells in non-treated cultures. Since the percentage of anti-HRP-positive cells did not differ between the Epo-treated and the control group, a stimulatory effect of Epo on M. sexta neural progenitor differentiation into mature neurons could not be demonstrated.

Given that anti-HRP antibodies labeled only small portions of cells that were positive for the other two neuronal markers anti-synapsin and anti-fasciclin II, the question arises whether this antibody is an appropriate marker of differentiated neurons in the M. sexta cell line. Assuming that neuron-specific Nervana protein is already expressed in cultured cells, but not glycosylated (Sun \& Salvaterra 1995), the absence of anti-HRP immunoreactivity would not necessarily indicate that all anti-HRP-negative cells have not yet been differentiated into neurons. In this respect, both anti-synapsin and anti-fasciclin II antibodies could be more appropriate markers of neuronal differentiation in 
the M. sexta cell line. Additionally, ELAV immunoreactivity can be used to detect neurons. Along with HRP, ELAV (embryonic lethal abnormal visual system) is the second best-characterized example of a pan-neuronal marker in Drosphila (Robinow \& White 1991) and anti-ELAV antibodies have already been used to label neurons in M. sexta (Swanson et al. 2005). However, since both immature and mature neurons express this pan-neuronal protein (Robinow \& White 1991) anti-ELAV immunoreactivity would not allow discrimination of differentiated from immature neurons. In this perspective, it would be advantageous to have defined markers that specify certain stages of neural development in M. sexta to test the influence of Epo on each of these stages more directly.

To sum up, Epo does not regulate neurogenesis in $M$. sexta in vitro by affecting the proliferation of neural progenitors. The data about Epo's differentiating potential are inconclusive, since it is debatable whether anti-HRP immunoreactivity can be used at all as a marker for neuronal differentiation in the $M$. sexta cell line.

\section{III.4.3 Epo does not support neurogenesis of optic lobe cells from nymphal} Locusta migratoria brains in vitro

While embryonic neurogenesis in L. migratoria has been documented already 40 years ago (Bate 1976), hardly any evidence of neurogenesis at later developmental stages in this species has been reported (Cayre et al. 1996). Even though earlier histological studies reported the persistence of neuroblasts in the optic lobes of different adult insect species (Johansson 1957; Panov 1960), postembryonic neurogenesis occurring in the optic lobes did not receive as much attention as the production of new Kenyon cells in mushroom bodies. It is conceivable that neuroblasts of most insect species, including D. melanogaster (Ito \& Hotta 1992), cease their proliferative activity with increasing numbers of divisions during early pupal development. However, a recent study using a modified lineage-labeling method unexpectedly provided evidence for 
continued neurogenesis in the adult optic lobes of D. melanogaster, which could be further stimulated by acute brain injury (Fernández-Hernández et al. 2013). Hence, insect optic lobes appear to be centers of neurogenesis which continues into adulthood.

To study the effects of Epo on insect neurogenesis during pupal development a primary culture of optic lobe cells from Locusta migratoria fourth instar nymphs was established. The influence of Epo on neurogenesis of primary cultured optic lobe cells was assessed by determining the portions of EdU-positive and anti-HRP-positive cells after 2 days of exposure to two different concentrations of rhEpo and comparisons with the portions of the same cell categories in untreated cell cultures. The obtained data do not support the involvement of Epo in the regulation of neurogenesis at the level of optic lobe neuroblast proliferation and their differentiation into neurons. Thus, it seems that Epo does not influence optic lobe neurogenesis in locust nymphs under normal conditions in vitro.

Since there are no immunocytochemical markers that label locust glia, it is not clear whether anti-HRP-negative cells in primary cultures of locust optic lobe cells represent glial cells or neuronal progenitors. Likewise, EdU-positive cells may represent either neuroblasts or glioblasts. Nevertheless, at least a certain portion of cells that divide can differentiate into neurons, as demonstrated by detection of several double EdU-/anti-HRP-positive cells in the culture. Therefore, comparison of the percentages of double EdU/anti-HRP-positive cells between treated and untreated cultures might be a better way to analyze the effect of Epo on neurogenesis. However, the quantification of this cell category is problematic, since in some cultures no double EdU/anti-HRPpositive cells were detected. Because of high variations between four individual experiments, apparently more trials are needed for an appropriate interpretation of the data. 


\section{III.4.4 The potential involvement of Epo in adult neurogenesis of Tribolium castaneum could not be evaluated}

One of the aims of the third part of PhD thesis was to establish an in vivo model system to study the potential effects of Epo on insect adult neurogenesis. As already mentioned, it has been widely acknowledged that adult neurogenesis is absent in a range of studied insect species. Many studies failed to demonstrate the presence of actively proliferating neuroblasts in adult brains of fruit flies (Ito \& Hotta 1992), honeybees (Fahrbach et al. 1995), butterflies (Nordlander \& Edwards 1970) and locusts (Cayre et al. 1996). Adult neurogenesis has been described for crickets (Cayre et al. 1994), cockroaches (Gu et al. 1999), migrant moths (Dufour \& Gadenne 2006) and several coleopteran species (Cayre et al. 1996). In the red flour beetle T. castaneum mushroom body neuroblasts undergoe continuous proliferation in the first two months of adult life, as demonstrated by BrdU staining (Zhao et al. 2008). Therefore, T. castaneum seemed to be suitable to address the question whether Epo regulates adult neurogenesis in insects.

Successful incorporation and reliable detection of EdU obtained in our in vitro studies of proliferation have encouraged the decision to apply the same assay for in vivo studies. However, in vivo demonstration of EdU incorporated into nuclei of dividing neuroblasts turned out to be more challenging. It was difficult to detect EdU/TAMRA-stained nuclear chromatin since other TAMRA-stained round objects of variable size, but smaller than the size of cell nuclei, were also observed in whole-mount brain samples. It is unlikely that the TAMRA stain has a higher affinity for cellular structures other than EdUincorporated DNA since "click" chemistry assures that the azide-coupled dye reacts only with EdU. Moreover, the distribution of unidentified TAMRA stained objects was not evenly throughout the whole brain tissue, which would be expected for unspecific staining. The staining pattern of these objects, however, resembled DAPI staining of late apoptotic cells, characterized by condensed nuclear chromatin. This indicates that some of the cells that incorporated EdU during $S$ phase of cell cycle might have afterwards entered 
apoptosis. A phenomenon of cellular senescence induced by EdU has been described in the literature. Cellular senescence is generally defined as an irreversible cell cycle arrest (Nakagawa \& Opitz 2007). Once incorporated into DNA, EdU can perturb cell cycle progression by slowing it down or arresting it completely, and induce DNA damage signaling that may lead subsequently to apoptosis (Kohlmeier et al. 2013; Zhao et al. 2013). The degree of the cell cycle progression impairment induced by EdU incorporation seems to depend on the cell type (Diermeier-Daucher et al. 2009). While some cells are retarded in their cell cycle progression others die soon after the cell cycle arrest (Kohlmeier et al. 2013).

In conclusion, EdU/TAMRA staining is not sufficiently reliable for proper in vivo identification and quantification of proliferating mushroom body neuroblasts in T. castaneum.

\section{III.4.5 Injury-induced Epo-mediated neurogenesis}

Epo-mediated regulation of adult neurogenesis in mammals has been demonstrated in most studies with respect to functional recovery after ischemic injury. The neurogenesis-stimulating effect of Epo in mammals can be attributed to the elevated expression of EpoR on neural progenitor cells induced by injury (Sirén et al. 2001b; Tsai et al. 2006; Ott et al. 2015). Only few studies investigated or showed the requirement of Epo for adult neurogenesis under physiological conditions. One study reported that large doses of systemically delivered rhEpo enhanced hippocampal neurogenesis in the normal adult mouse brain under basal conditions in vivo. This effect was however transient, since Epo did not support long-term survival and integration of newborn neurons into the existing neural circuitry (Ransome \& Turnley 2007). As shown by Hassouna et al., the maintenance of newly generated pyramidal neurons induced by Epo treatment was supported in healthy young mice that were continuously cognitively challenged, but not in mice without cognitive challenge (Hassouna et al. 2016). This suggests that 
newly generated neurons need to be "used“ in order to be sustained. In my experiments the potential effects of Epo on insect neurogenesis were investigated under normal conditions, in the absence of strong stressor or cognitive challenge. Previous studies on locusts in vitro (see chapter I) have shown that under normoxic conditions the effects of Epo on neuronal survival may not be significant. Similarly, neurogenic effects of Epo may not easily be demonstrated under conditions that do not require integration and "usage" of newborn neurons. It would be worth to establish an insect model of acute brain injury and/or a learning paradigm in which the neurogenic potential of Epo can be more explicitly studied. 


\section{CONCLUSIONS AND OUTLOOK}

This study characterized several functional properties of a neuroprotective Epobinding receptor on locust neurons. (1) The receptor is associated with JAK, whose activity and subsequent STAT activation are required for anti-apoptotic effects of rhEpo; (2) Upon rhEpo binding the ligand-receptor complex is internalized by endocytosis; (3) The receptor shares similarities with the mammalian neuroprotective Epo receptor, since both are activated by the human Epo splice variant EV3, which does not stimulate the homodimeric hematopoietic EpoR. In order to structurally identify the neuroprotective Epo receptor in insects future studies should apply direct molecular approaches. Such approaches might require the application of genetic tools. However, the genetically best accessible species Drosophila melanogaster is not suitable for these prospective studies, since Epo-mediated neuroprotection was absent in this species (Pompe 2013). Furthermore, comparison of gene orthologues across animal phyla showed that $D$. melanogaster has lost a large number of ancestral genes common to vertebrates and more typical insects (Wyder et al. 2007). In this respect, Tribolium castaneum emerges as a promising alternative species for "receptor studies" that is amenable to genetic manipulations. A candidate receptor in T. castaneum, an orthologue of a human cytokine receptor, is currently studied for its involvement in Epo-mediated neuroprotection in insects and mammals.

Even though studies with the species Locusta migratoria are limited by the narrow scope of genetic approaches that can be applied to identify insect Epobinding receptors, alternative approaches to study ligand-receptor interactions are emerging lately and some of them can be used in this species, including fluorescence-based ligand binding assays. For example, one can study the binding affinity of fluorescently labeled rhEpo to a potential receptor in locust neurons by means of microscale thermophoresis (MST) (Wienken et al. 2010). Intermolecular fluorescence resonance energy transfer (FRET) from a donor fluorophore associated with a ligand to an acceptor fluorophore connected with 
a receptor can be used as an alternative approach to study interaction between rhEpo and potential receptor candidates (De Jong et al. 2005; Ward \& Milligan 2014).

In conclusion, this PhD study provided some important information about the cellular mechanisms underlying Epo-mediated neuroprotection in insects. Furthermore, it pointed out those underlying mechanisms that are similar for vertebrates and insects. Future studies on insects may provide important insights into the evolution of tissue-protective Epo-like signaling that still plays an indispensable role in higher vertebrates. The knowledge gained from „Epo studies" on insects may have translational potential in the future, considering that successful separation of erythropoietic from tissue-protective properties of Epo is a prerequisite for its safe application as a therapeutic agent for treatment of nervous system disorders. 


\section{SUMMARY}

The cytokine erythropoietin (Epo) initiates adaptive cellular responses to a variety of mechanical and physiological insults in various non-hematopoietic mammalian tissues including the nervous system. Previous studies on insects demonstrated neuroprotective and regenerative effects of recombinant human Epo (rhEpo) in acridid grasshoppers in vitro and in vivo, similar to those in mammalian nervous system. This suggested that Epo-like signaling involved in tissue protection could represent an ancient cell-protective system shared by vertebrates and invertebrates that was later adopted for erythropoiesis in the vertebrate lineage. This $\mathrm{PhD}$ thesis provides further evidence for a prevertebrate evolution of a tissue-protective Epo-like signaling system.

In the first part of the thesis I studied intracellular transduction pathways involved in Epo-mediated protection of locust brain neurons. I demonstrate that rhEpo effectively rescues primary cultured locust brain neurons from apoptotic cell death induced by hypoxia or the chemical compound H-7. The protective effects of rhEpo on locust brain neurons were abolished by the Janus kinase (JAK) inhibitor AG-490 and signal transducer and activator of transcription (STAT) inhibitor sc-355797. In contrast, the phosphoinositol-3-kinase (PI3K) inhibitor LY294002 and an inhibitor of nuclear factor kappa-light-chainenhancer of activated B cells (NF-kB) PDTC did not prevent rhEpo-mediated neuroprotection. The results indicate that rhEpo mediates the protection of locust brain neurons through interference with apoptotic pathways by the activation of a JAK-associated receptor and STAT transcription factors.

In the second part of the thesis I characterized some functional properties of the locust Epo-binding receptor. Using the fluorescent dye FM1-43 to quantify endocytotic activity I demonstrated that binding of rhEpo to a surface receptor initiates endocytotic internalization of the ligand-receptor complex. The results were confirmed by the detection of fluorescently labeled rhEpo in endocytotic vesicles. Epo-stimulated endocytosis has been demonstrated in vertebrate 
erythroid progenitor cells but so far not in any other tissue outside the erythropoietic system. In another series of experiments I could show that the neuroprotective but non-erythropoietic human Epo splice variant EV3 protected locust neurons from hypoxia-induced apoptosis with equal potency as rhEpo. The shared neuroprotective potency of EV3 in mammals and insects in the absence of erythropoietic effects suggests a greater similarity of the unidentified nervous erythropoietin-binding receptors across phyla than between mammalian hematopoietic and neuroprotective receptors.

In the third part of the thesis I studied the potential role of rhEpo in the regulation of insect neurogenesis using three different preparations including a permanent cell line from a moth, a brain region with known neurogenic activity in a beetle and cells from a developing brain region with proliferative activity in the locust. With the tools and conditions applied, I found no evidence for an involvement of Epo in the regulation of neurogenesis neither at the level of progenitor cells proliferation nor at the level of neuronal differentiation.

In summary, the present study demonstrated three important characteristics of Epo-like neuroprotective signaling in locust brain neurons that underline the similarity of mechanisms involved in insect and mammalian Epo-mediated neuroprotection. The involvement of similar transduction pathways in Epomediated neuroprotection, the endocytosis of Epo following its binding to a receptor and the capacity of EV3 to stimulate neuroprotection in mammals and insects, indicate that an Epo/Epo receptor-like signaling system with high structural and functional similarity exists in both groups of animals and may have originally evolved to provide tissue protection against various types of stressors. 


\section{REFERENCES}

Adamcio, B., Sargin, D., Stradomska, A., Medrihan, L., Gertler, C., Theis, F. et al., 2008. Erythropoietin enhances hippocampal long-term potentiation and memory. BMC Biology, 6, p.37.

Adamo, S.A., 2008. Bidirectional connections between the immune system and the nervous system in insects. In N. E. Beckage, ed. Insect Immunology. Elsevier, pp. 129-149. Available at: http:/ / www.sciencedirect.com/science/book/9780123739766 [Accessed July 7, 2016].

Agaisse, H., Petersen, U. M., Boutros, M., Mathey-Prevot, B., \& Perrimon, N., 2003. Signaling role of hemocytes in Drosophila JAK/STAT-dependent response to septic injury. Developmental Cell, 5(3), pp.441-450.

Agnello, D., Bigini, P., Villa, P., Mennini, T., Cerami, A., Brines, M. L., \& Ghezzi, P., 2002. Erythropoietin exerts an anti-inflammatory effect on the CNS in a model of experimental autoimmune encephalomyelitis. Brain research, 952(1), pp.128-34.

Aparicio, S., Chapman, J., Stupka, E., Putnam, N., Chia, J.M., Dehal, P. et al., 2002. Whole-genome shotgun assembly and analysis of the genome of Fugu rubripes. Science, 297(5585), pp.1301-1310.

Arbouzova, N.I. \& Zeidler, M.P., 2006. JAK/STAT signalling in Drosophila: insights into conserved regulatory and cellular functions. Development, 133(14), pp.2605-2616.

Baeuerle, P. A. \& Baltimore, D., 1988. IкB: a specific inhibitor of the NF-кB transcription factor. Science, 242(4878), pp.540-546.

Bao, H., Jacobs-Helber, S. M., Lawson, A. E., Penta, K., Wickrema, A., \& Sawyer, S. T., 1999. Protein kinase B (c-Akt), phosphatidylinositol 3-kinase, and STAT5 are activated by erythropoietin (EPO) in HCD57 erythroid cells but are constitutively active in an EPO-independent, apoptosis-resistant subclone (HCD57-SREI cells). Blood, 93(11), pp.3757-73.

Basca, C.A., Talos, M. \& Brad, R., 2005. Randomized Hough transform for ellipse detection with result clustering. In EUROCON 2005 - The International Conference on "Computer as a Tool." IEEE, pp. 1397-1400.

Basith, S., Manavalan, B., Gosu, V., \& Choi, S., 2013. Evolutionary, structural and functional interplay of the IкB family members. PLoS ONE, 8(1), p.e54178.

Basquin, C. \& Sauvonnet, N., 2013. Phosphoinositide 3-kinase at the crossroad between endocytosis and signaling of cytokine receptors. Communicative and Integrative Biology, 6(4), p.e24243. 
Bastiani, M.J., Harrelson, A. L., Snow, P. M., \& Goodman, C. S., 1987.

Expression of fasciclin-I and fasciclin-II glycoproteins on subsets of axon pathways during neuronal development in the grasshopper. Cell, 48(5), pp.745-755.

Bate, C.M., 1976. Embryogenesis of an insect nervous system I . A map of the thoracic and abdominal neuroblasts in Locusta migratoria. Journal of Embryology and Experimental Morphology, 35, pp.107-123.

Bazan, J.F., 1990. Structural design and molecular evolution of a cytokine receptor superfamily. Proceedings of the National Academy of Sciences of the United States of America, 87(18), pp.6934-6938.

Bazinet, C., Katzen, A. L., Morgan, M., Mahowald, A. P., \& Lemmon, S. K., 1993. The Drosophila clathrin heavy chain gene: Clathrin function is essential in a multicellular organism. Genetics, 134(4), pp.1119-1134.

Becker, V., Schilling, M., Bachmann, J., Baumann, U., Raue, A., Maiwald, T. et al., 2010. Covering a broad dynamic range: information processing at the erythropoietin receptor. Science, 328(5984), pp.1404-1408.

Benjamini, J. \& Hochberg, Y., 1995. Controlling the false discovery rate: a practical and powerful approach to multiple testing. Journal of the Royal Statistical Society, B, 57(1), pp.289-300.

Bergmann, A., Agapite, J. \& Steller, H., 1998. Mechanisms and control of programmed cell death in invertebrates. Oncogene, 17, pp.3215-23.

Bernaudin, M., Marti, H. H., Roussel, S., Divoux, D., Nouvelot, A., Mackenzie, E. T., \& Petit, E., 1999. A potential role for erythropoetin in focal permanent cerebral ischemia in mice. J Cereb Blood Flow Metab, 19(6), pp.643-651.

Bernaudin, M., Bellail, A., Marti, H. H., Yvon, A., Vivien, D., Duchatelle, I. et al., 2000. Neurons and astrocytes express EPO mRNA: Oxygen-sensing mechanisms that involve the redox-state of the brain. Glia, 30(3), pp.271278.

Bertrand, N., Castro, D.S. \& Guillemot, F., 2002. Proneural genes and the specification of neural cell types. Nature Reviews Neuroscience, 3(7), pp.517530.

Bina, S. \& Zeidler, M., 2009. JAK-STAT Pathway in Disease. In A. Stephanou, ed. Landes Bioscience, University of College London, London UK, pp. 24 42. Available at: http://www.ncbi.nlm.nih.gov/books/NBK6034/.

Binari, R. \& Perrimon, N., 1994. Stripe-specific regulation of pair-rule genes by hopscotch, a putative Jak family tyrosine kinase in Drosophila. Genes $\mathcal{E}$ Development, 8(3), pp.300-312. 
Boissel, J., Lee, W., Presnell, S., Cohen, F., \& Bunn, H., 1993. Erythropoietin structure-function relationships. Mutant proteins that test a model of tertiary structure. J Biol Chem, 268(21), pp.15983-93.

Bonnas, C.B., 2009. Identification of erythropoietin isoforms and evaluation of their biological importance. PhD Thesis. Medical Faculty Charité, Berlin. Available at: http:/ / www.diss.fuberlin.de/diss/receive/FUDISS_thesis_000000009488.

Booker, R. \& Truman, J.W., 1987. Postembryonic neurogenesis in the CNS of the tobacco hornworm, Manduca sexta. I. Neuroblast arrays and the fate of their progeny during metamorphosis. The Journal of comparative neurology, 255(4), pp.548-559.

Boulay, J.L. \& Paul, W.E., 1992. The interleukin-4-related lymphokines and their binding to hematopoietin receptors. Journal of Biological Chemistry, 267(29), pp.20525-20528.

Boulay, J.L., Shea, J.J.O. \& Paul, W.E., 2003. Molecular phylogeny within type I cytokines and their cognate receptors. Immunity, 19, pp.159-163.

Brines, M.L., Ghezzi, P., Keenan, S., Agnello, D., de Lanerolle, N. C., Cerami, C. et al., 2000. Erythropoietin crosses the blood-brain barrier to protect against experimental brain injury. Proceedings of the National Academy of Sciences of the United States of America, 97(19), pp.10526-31.

Brines, M., Grasso, G., Fiordaliso, F., Sfacteria, A., Ghezzi, P., Fratelli, M. et al., 2004. Erythropoietin mediates tissue protection through an erythropoietin and common $\beta$-subunit heteroreceptor. Proceedings of the National Academy of Sciences of the United States of America, 101(41), pp.14907-14912.

Brines, M. \& Cerami, A., 2005. Emerging biological roles for erythropoietin in the nervous system. Nature reviews. Neuroscience, 6(6), pp.484-94.

Brines, M., Patel, N. S. A., Villa, P., Brines, C., Mennini, T., De Paola, M. et al., 2008. Nonerythropoietic, tissue-protective peptides derived from the tertiary structure of erythropoietin. Proceedings of the National Academy of Sciences of the United States of America, 105(31), pp.10925-10930.

Brines, M., 2014. Discovery of a master regulator of injury and healing: tipping the outcome from damage toward repair. Molecular Medicine, 20(Supplement 1), pp.10-16.

Broudy, V.C., Lin, N., Brice, M., Nakamoto, B., \& Papayannopoulou, T., 1991. Erythropoietin receptor characteristics on primary human erythroid cells. Blood, 77, pp.2583-2590.

Brown, S., Hu, N. \& Hombría, J.C.G., 2001. Identification of the first invertebrate interleukin JAK/STAT receptor, the Drosophila gene domeless. Current Biology, 11(21), pp.1700-1705. 
Broxmeyer, H.E., 2013. Erythropoietin: multiple targets, actions, and modifying influences for biological and clinical consideration. Journal of Experimental Medicine, 210(2), pp.205-208.

Brunn, G.J., Williams, J., Sabers, C., Wiederrecht, G., Lawrence, J. C., \& Abraham, R. T., 1996. Direct inhibition of the signaling functions of the mammalian target of rapamycin by the phosphoinositide 3-kinase inhibitors, wortmannin and LY294002. The EMBO journal, 15(19), pp.52565267.

Brust, M.L., Hoback, W.W. \& Wright, R.J., 2007. Immersion tolerance in rangeland grasshoppers (Orthoptera: Acrididae). Journal of Orthoptera Research, 16(2), pp.135-138.

Buchmann, K., 2014. Evolution of innate immunity: clues from invertebrates via fish to mammals. Frontiers in Immunology, 5, p.459.

Bulut, G.B., Sulahian, R., Ma, Y., Chi, N. W., \& Huang, L. J. S., 2011. Ubiquitination regulates the internalization, endolysosomal sorting, and signaling of the erythropoietin receptor. Journal of Biological Chemistry, 286(8), pp.6449-6457.

Bulut, G.B., Sulahian, R., Yao, H., \& Huang, L. J., 2013. Cbl ubiquitination of p85 is essential for Epo-induced EpoR endocytosis. Blood, 122(24), pp.3964-72.

Bunn, H.F., 2013. Erythropoietin. Cold Spring Harbor Perspectives in Medicine, 3, p.a011619.

Byts, N. \& Sirén, A.-L., 2009. Erythropoietin: a multimodal neuroprotective agent. Experimental $\mathcal{E}$ Translational Stroke Medicine, 1(1), p.4.

Camidge, D.R. \& Pearse, B.M.F., 1994. Cloning of Drosophila $\beta$-adaptin and its localization on expression in mammalian cells. Journal of Cell Science, 107, pp.709-718.

Cantrell, D.A., 2001. Phosphoinositide 3-kinase signalling pathways. Journal of cell science, 114(8), pp.1439-1445.

Cayre, M., Strambi, C. \& Strambi, A., 1994. Neurogenesis in an adult insect brain and its hormonal control. Nature, 368(6466), pp.57-59.

Cayre, M., Strambi, C., Charpin, P., Augier, R., Meyer, M. R., Edwards, J. S., \& Strambi, A., 1996. Neurogenesis in adult insect mushroom bodies. Journal of Comparative Neurology, 371(2), pp.300-310.

Cayre, M., Scotto-Lomassese, S., Malaterre, J., Strambi, C., \& Strambi, A., 2007. Understanding the regulation and function of adult neurogenesis: contribution from an insect model, the house cricket. Chemical Senses, 32(4), pp.385-395. 
Chandel, N.S., Maltepe, E., Goldwasser, E., Mathieu, C. E., Simon, M. C., \& Schumacker, P. T., 1998. Mitochondrial reactive oxygen species trigger hypoxia-induced transcription. Proc. Natl. Acad. Sci. USA, 95, pp.1171511720.

Chandel, N.S., McClintock, D. S., Feliciano, C. E., Wood, T. M., Melendez, J. A., Rodriguez, A. M., \& Schumacker, P. T., 2000. Reactive Oxygen Species Generated at Mitochondrial Complex III Stabilize Hypoxia-inducible Factor-1 during Hypoxia. A mechanism of $\mathrm{O} 2$ sensing. Journal of Biological Chemistry, 275(33), pp.25130-25138.

Chateauvieux, S., Grigorakaki, C., Morceau, F., Dicato, M., \& Diederich, M., 2011. Erythropoietin, erythropoiesis and beyond. Biochemical Pharmacology, 82(10), pp.1291-1303.

Cheetham, J.C., Smith, D. M., Aoki, K. H., Stevenson, J. L., Hoeffel, T. J., Syed, R. S. et al., 1998. NMR structure of human erythropoietin and a comparison with its receptor bound conformation. Nature structural biology, 5(10), pp.861-866.

Chen, M.S., Obar, R. A, Schroeder, C. C., Austin, T. W., Poodry, C. A, Wadsworth, S. C., \& Vallee, R. B., 1991. Multiple forms of dynamin are encoded by shibire, a Drosophila gene involved in endocytosis. Nature, 351(6327), pp.583-586.

Chen, G., Shi, J. X., Hang, C. H., Xie, W., Liu, J., \& Liu, X. et al., 2007a. Inhibitory effect on cerebral inflammatory agents that accompany traumatic brain injury in a rat model: A potential neuroprotective mechanism of recombinant human erythropoietin (rhEPO). Neuroscience Letters, 425(3), pp.177-182.

Chen, Z.Y., Asavaritikrai, P., Prchal, J. T., \& Noguchi, C. T., 2007b. Endogenous erythropoietin signaling is required for normal neural progenitor cell proliferation. Journal of Biological Chemistry, 282(35), pp.25875-25883.

Chong, Z.Z., Kang, J.Q. \& Maiese, K., 2002. Erythropoietin is a novel vascular protectant through activation of AKt1 and mitochondrial modulation of cysteine proteases. Circulation, 106(23), pp.2973-2979.

Chong, Z.Z., Kang, J.Q. \& Maiese, K., 2003. Erythropoietin fosters both intrinsic and extrinsic neuronal protection through modulation of microglia, Akt1, Bad, and caspase-mediated pathways. British Journal of Pharmacology, 138(6), pp.1107-1118.

Chong, Z.Z., Li, F. \& Maiese, K., 2005. Activating Akt and the brain's resources to drive cellular survival and prevent inflammatory injury. Histol Histopathol., 20(1), pp.299-315.

Chou, C.F., Tohari, S., Brenner, S., \& Venkatesh, B., 2004. Erythropoietin gene from a teleost fish, Fugu rubripes. Blood, 104(5), pp.1498-1503. 
Chu, C.Y. et al. Cheng, C.H., Yang, C.H., \& Huang, C. J., 2008. Erythropoietins from teleosts. Cellular and Molecular Life Sciences, 65(22), pp.3545-3552.

Chu, C.Y., Cheng, C.H., Chen, G.D., Chen, Y.C., Hung, C.C., Huang, K.Y., \& Huang, C.J., 2007. The zebrafish erythropoietin: Functional identification and biochemical characterization. FEBS Letters, 581(22), pp.4265-4271.

Clark, K.D., Garczynski, S. F., Arora, A., Crim, J. W., \& Strand, M. R., 2004. Specific residues in plasmatocyte-spreading peptide are required for receptor binding and functional antagonism of insect immune cells. Journal of Biological Chemistry, 279(32), pp.33246-33252.

Collino, M., Thiemermann, C., Cerami, A., \& Brines, M., 2015. Flipping the molecular switch for innate protection and repair of tissues: Long-lasting effects of a non-erythropoietic small peptide engineered from erythropoietin. Pharmacology $\mathcal{E}$ therapeutics, 151, pp.32-40.

Constantinescu, S., Ghaffari, S. \& Lodish, H., 1999. The erythropoietin receptor: structure, activation and intracellular signal transduction. Trends in endocrinology and metabolism, 10(1), pp.18-23.

Constantinescu, S.N., Keren, T., Socolovsky, M., Nam, H., Henis, Y. I., \& Lodish, H. F., 2001. Ligand-independent oligomerization of cell-surface erythropoietin receptor is mediated by the transmembrane domain. Proceedings of the National Academy of Sciences of the United States of America, 98(8), pp.4379-4384.

Cooper, D.M., Granville, D.J. \& Lowenberger, C., 2009. The insect caspases. Apoptosis, 14(3), pp.247-256.

Corwin, H.L., Gettinger, A., Fabian, T.C., May, A., Pearl, R.G., Heard, S. et al., 2007. Efficacy and safety of epoetin alfa in critically ill patients. The New England Journal of Medicine, 357(10), pp.965-976.

Cottet, M., Faklaris, O., Zwier, J. M., Trinquet, E., Pin, J.P., \& Durroux, T., 2011. Original Fluorescent Ligand-Based Assays Open New Perspectives in GProtein Coupled Receptor Drug Screening. Pharmaceuticals, 4(12), pp.202214.

Debeljak, N., Solár, P. \& Sytkowski, A.J., 2014. Erythropoietin and cancer: the unintended consequences of anemia correction. Frontiers in Immunology, 5, p.563.

Dekanty, A., Romero, N. M., Bertolin, A. P., Thomas, M. G., Leishman, C. C., Perez-Perri, J. I. et al., 2010. Drosophila genome-wide RNAi screen identifies multiple regulators of HIF-dependent transcription in hypoxia. PLoS Genetics, 6(6), p.e1000994. 
Diermeier-Daucher, Clarke, S. T., Hill, D., Vollmann-Zwerenz, A., Bradford, J. A., \& Brockhoff, G., 2009. Cell type specific applicability of 5-ethynyl-2'deoxyuridine (EDU) for dynamic proliferation assessment in flow cytometry. Cytometry Part A, 75A, pp.535-546.

Dieudonne, F.X., Sévère, N., Biosse-Duplan, M., Weng, J. J., Su, Y., \& Marie, P. J., 2013. Promotion of osteoblast differentiation in mesenchymal cells through Cbl-mediated control of STAT5 activity. Stem Cells, 31(7), pp.13401349.

Digicaylioglu, M., Bichet, S., Marti, H. H., Wenger, R. H., Rivas, L. A, Bauer, C., \& Gassmann, M, 1995. Localization of specific erythropoietin binding sites in defined areas of the mouse brain. Proceedings of the National Academy of Sciences of the United States of America, 92(9), pp.3717-20.

Digicaylioglu, M. \& Lipton, S.A., 2001. Erythropoietin-mediated neuroprotection involves cross-talk between Jak2 and NF-kappaB signalling cascades. Nature, 412(6847), pp.641-647.

Digicaylioglu, M., Garden, G., Timberlake, S., Fletcher, L., \& Lipton, S. A., 2004. Acute neuroprotective synergy of erythropoietin and insulin-like growth factor I. Proceedings of the National Academy of Sciences of the United States of America, 101(26), pp.9855-9860.

Dornan, S., Jackson, A.P. \& Gay, N.J., 1997. a-adaptin, a marker for endocytosis, is expressed in complex patterns during Drosophila development. Molecular Biology of the Cell, 8, pp.1391-1403.

Dowling, J.E., 2007. The great brain debate: nature or nurture?, Princeton University Press. Available at:

https:/ / books.google.de/books?id=bRZ2UpypAkEC\&pg=PA112\&lpg=PA $112 \& d q=$ Deprived + of + oxygen,+ mammalian + neurons + die + in + just $+a+f e w$ + minutes\&source=bl\&ots=LFRdfT8S7L\&sig=DDN9SFNfaa8MQla87-

X4x645UEQ\&hl=sr\&sa=X\&ved=0ahUKEwiytp33snLAhVEGA8KHRx0CdUQ6AEIHTAA\#v=onepage.

Dufour, M.C. \& Gadenne, C., 2006. Adult neurogenesis in a moth brain. Journal of Comparative Neurology, 495(5), pp.635-643.

Dushay, M.S., Asling, B. \& Hultmark, D., 1996. Origins of immunity: Relish, a compound Rel-like gene in the antibacterial defense of Drosophila. Proceedings of the National Academy of Sciences of the United States of America, 93(19), pp.10343-10347.

Ehrenreich, H., Hasselblatt, M., Dembowski, C., Cepek, L., Lewczuk, P. et al., 2002. Erythropoietin therapy for acute stroke is both safe and beneficial. Molecular medicine (Cambridge, Mass.), 8(8), pp.495-505. 
Ehrenreich, H., Fischer, B., Norra, C., Schellenberger, F., Stender, N., Stiefel, M. et al., 2007. Exploring recombinant human erythropoietin in chronic progressive multiple sclerosis. Brain : a journal of neurology, 130(Pt 10), pp.2577-88.

Ehrenreich, H., Weissenborn, K., Prange, H., Schneider. D., Weimar, C., Wartenberg, K. et al., 2009. Recombinant human erythropoietin in the treatment of acute ischemic stroke. Stroke; a journal of cerebral circulation, 40(12), pp.e647-56.

Eide, P.E., Caldwell, J.M. \& Marks, E.P., 1975. Establishment of Two Cell Lines from Embryonic Tissue of the Tobacco Hornworm, Manduca sexta ( L .). In Vitro, 11(6), pp.395-399. Available at: http:/ / www.jstor.org/stable/4291886.

Elliott, S., Sinclair, A., Collins, H., Rice, L., \& Jelkmann, W. et al., 2014. Progress in detecting cell-surface protein receptors: the erythropoietin receptor example. Annals of hematology, 93(2), pp.181-92.

Elmore, S., 2007. Apoptosis: a review of programmed cell death. Toxicologic pathology, 35(4), pp.495-516.

Erbayraktar, S., Grasso, G., Sfacteria, A., Xie, Q., Coleman, T., Kreilgaard, M. et al., 2003. Asialoerythropoietin is a nonerythropoietic cytokine with broad neuroprotective activity in vivo. Proceedings of the National Academy of Sciences, 100(11), pp.6741-6746.

Ernst, M.D., 2004. Permutation methods: a basis for exact inference. Statistical Science, 19(4), pp.676-685.

ExPASy Bioinformatics Resource Portal, 2011. ProtParam tool [Online]. Available at: www.expasy.ch/tools/protparam.html [Accessed August 11, 2015].

Fahrbach, S.E., Strande, J.L. \& Robinson, G.E., 1995. Neurogenesis is absent in the brains of adult honey bees and does not explain behavioral neuroplasticity. Neuroscience Letters, 197(2), pp.145-148.

Farris, S.M., 2005. Developmental organization of the mushroom bodies of Thermobia domestica (Zygentoma, Lepismatidae): Insights into mushroom body evolution from a basal insect. Evolution and Development, 7(2), pp.150159.

Fernández-Hernández, I., Rhiner, C. \& Moreno, E., 2013. Adult neurogenesis in Drosophila. Cell Reports, 3(6), pp.1857-1865.

Firth, J.D., Ebert, B.L. \& Ratcliffe, P.J., 1995. Hypoxic regulation of lactate dehydrogenase A. Journal of Biological Chemistry, 270(36), pp.21021-21027. 
Fischer, J.A., Eun, S.H. \& Doolan, B.T., 2006. Endocytosis, endosome trafficking, and the regulation of Drosophila development. Annual Review of Cell and Developmental Biology, 22(1), pp.181-206.

Fisher, R.A., 1954. Statistical methods for research workers 12th editi., Edinburg: Oliver and Boyd.

Franke, T.F., Tartof, K.D. \& Tsichlis, P.N., 1994. The SH2-like Akt homology $(\mathrm{AH})$ domain of c-akt is present in multiple copies in the genome of vertebrate and invertebrate eucaryotes. Cloning and characterization of the Drosophila melanogaster c-akt homolog Dakt1. Oncogene, 9(1), pp.141-8.

Gaffield, M.A. \& Betz, W.J., 2006. Imaging synaptic vesicle exocytosis and endocytosis with FM dyes. Nature protocols, 1(6), pp.2916-2921.

Galletta, B.J. \& Cooper, J.A., 2009. Actin and endocytosis: mechanisms and phylogeny. Current Opinion in Cell Biology, 21(1), pp.20-27.

Ghosh, S., May, M.J. \& Kopp, E.B., 1998. NF-кB and Rel proteins: Evolutionarily conserved mediators of immune responses. Annual review of immunology, 16, pp.225-260.

Giaccia, A.J., Simon, M.C. \& Johnson, R., 2004. The biology of hypoxia: The role of oxygen sensing in development, normal function, and disease. Genes and Development, 18, pp.2183-2194.

Gilbert-Kawai, E. T. \& Wittenberg ,M.D., 2014. Beer-Lambert Law. In: Essential Equations for Anaes thesia.pp. 43-44 [Online]. Cambridge: Cambridge University Press. Available at: Cambridge Books Online http:/ / dx.doi.org/10.1017/CBO9781139565387.023 [Accessed January 26, 2016]

Gocht, D., Wagner, S. \& Heinrich, R., 2009. Recognition, Presence, and Survival of Locust Central Nervous Glia In Situ and In Vitro. Microscopy Research and Technique, 72(5), pp.385-397.

Gordon, J., Amini, S. \& White, M.K., 2013. General overview of neuronal cell culture. In Neuronal Cell Culture: Methods and Protocols. Springer, pp.35-44. Available at: http:/ / link.springer.com/10.1007/978-1-62703-640-5.

Gorr, T.A., Gassmann, M. \& Wappner, P., 2006. Sensing and responding to hypoxia via HIF in model invertebrates. Journal of Insect Physiology, 52(4), pp.349-364.

Green, D.R., 1998. Apoptic pathways: The roads to ruin. Cell, 94(6), pp.695-698.

Greenlee, K.J. \& Harrison, J.F., 2004. Development of respiratory function in the American locust Schistocerca americana. I. Across-instar effects. The Journal of Experimental Biology, 207, pp.497-508. 
Grenningloh, G., Rehm, E.J. \& Goodman, C.S., 1991. Genetic analysis of growth cone guidance in Drosophila: fasciclin II functions as a neuronal recognition molecule. Cell, 67(1), pp.45-57.

Groppe, D.M., Urbach, T.P.\& Kutas, M., 2011. Mass univariate analysis of event-related brain potentials / fields I : A critical tutorial review. Psychophysiology, 48, pp.1711-1725.

Gross, A.W. \& Lodish, H.F., 2006. Cellular trafficking and degradation of erythropoietin and novel erythropoiesis stimulating protein (NESP). Journal of Biological Chemistry, 281, pp.2024-2032.

Gu, S.H., Tsia, W. H., Chiang, A. S., \& Chow, Y. S., 1999. Mitogenic effects of 20hydroxyecdysone on neurogenesis in adult mushroom bodies of the cockroach, Diploptera punctata. Journal of neurobiology, 39(2), pp.264-74.

Gu, S.H., Yeh, W.L., Young, S.C., Lin, P.L., \& Li, S., 2012. TOR signaling is involved in PTTH-stimulated ecdysteroidogenesis by prothoracic glands in the silkworm, Bombyx mori. Insect biochemistry and molecular biology, 42(4), pp.296-303.

Haddad, J.J.E., Olver, R.E. \& Land, S.C., 2000. Antioxidant/pro-oxidant equilibrium regulates HIF-1a and NF-kB redox sensitivity: Evidence for inhibition by glutathione oxidation in alveolar epithelial cells. Journal of Biological Chemistry, 275(28), pp.21130-21139.

Hahn, N., 2014. Erythropoietin-mediated neuroprotection and potential contribution of the cytokine receptor CRLF3 in insects. MSc Thesis. Georg-AugustUniversity Göttingen.

Hamshou, M., Van Damme, E. J. M., Vandenborre, G., Ghesquière, B., Trooskens, G., Gevaert, K., \& Smagghe, G., 2012. GalNAc/Gal-binding Rhizoctonia solani agglutinin has antiproliferative activity in Drosophila melanogaster S2 cells via MAPK and JAK/STAT signaling. PloS one, 7(4), p.e33680.

Handl, H.L., Vagner, J., Yamamura, H. I., Hruby, V. J., \& Gillies, R. J., 2005. Development of a lanthanide-based assay for detection of receptor-ligand interactions at the $\delta$-opioid receptor. Analytical Biochemistry, 343(2), pp.299307.

Hardman, K. \& Ainsworth, C., 1972. Structure of concanavalin A at 2.4-Ang resolution. Biochemistry, 11(26), pp.4910-4919.

Harrelson, A. L. \& Goodman, C.S., 1988. Growth cone guidance in insects: fasciclin II is a member of the immunoglobulin superfamily. Science, 242(4879), pp.700-708. 
Harrison, D. A., McCoon, P. E., Binari, R., Gilman, M., \& Perrimon, N., 1998. Drosophila unpaired encodes a secreted protein that activates the JAK signaling pathway. Genes and Development, 12(20), pp.3252-3263.

Hassouna, I., Ott, C., Wüstefeld, L., Offen, N., Neher, R. A., Mitkovski, M. et al., 2016. Revisiting adult neurogenesis and the role of erythropoietin for neuronal and oligodendroglial differentiation in the hippocampus. Molecular Psychiatry, pp.1-16. Available at: http:/ / www.nature.com/doifinder/10.1038/mp.2015.212.

Hayakawa, Y., 1991. Structure of a growth-blocking peptide present in parasitized insect hemolymph. Journal of Biological Chemistry, 266(13), pp.7982-7984.

Hayakawa, Y. \& Noguchi, H., 1998. Growth-blocking peptide expressed in the insect nervous system. Cloning and functional characterization. European Journal of Biochemistry, 253(3), pp.810-816.

Hémar, A., Lieb, M., Subtil, A., DiSanto, J. P., \& Dautry-Varsat, A., 1994. Endocytosis of the $\beta$ chain of interleukin-2 receptor requires neither interleukin-2 nor the $\gamma$ chain. European journal of immunology, 24(9), pp.1951-1955.

Hoback, W.W. \& Stanley, D.W., 2001. Insects in hypoxia. Journal of insect physiology, 47(6), pp.533-542.

Hochachka, P.W., Nener, J. C., Hoar, J., Saurez, R. K., \& Hand, S. C., 1993. Disconnecting metabolism from adenylate control during extreme oxygen limitation. Canadian Journal of Zoology, 71(6), pp.1267-1270.

Hoffmann, A. \& Baltimore, D., 2006. Circuitry of nuclear factor кB signaling. Immunological reviews, 210, pp.171-186.

Hombría, J.C.G., Brown, S., Häder, S., \& Zeidler, M. P., 2005. Characterisation of Upd2, a Drosophila JAK/STAT pathway ligand. Developmental Biology, 288(2), pp.420-433.

Hou, X.S., Melnick, M.B. \& Perrimon, N., 1996. Marelle acts downstream of the Drosophila HOP/JAK kinase and encodes a protein similar to the mammalian STATs. Cell, 84(3), pp.411-419.

Huang, H.-R., Chen, Z. J., Kunes, S., Chang, G.-D., \& Maniatis, T., 2010. Endocytic pathway is required for Drosophila Toll innate immune signaling. Proceedings of the National Academy of Sciences of the United States of America, 107(18), pp.8322-8327.

Ip, Y.T., Reach, M., Engstrom, Y., Kadalayil, L., Cai, H., Gonzalez-Crespo, S. et al, 1993. Dif, a dorsal-related gene that mediates an immune response in Drosophila. Cell, 75(4), pp.753-763. 
Ito, K. \& Hotta, Y., 1992. Proliferation pattern of postembryonic neuroblasts in the brain of Drosophila melanogaster. Developmental biology, 149(1), pp.134148.

Jackson, D., Stein, M., Voss, H., \& Brock, S., 2012. Tissue protective erythropoietin receptor (nepor) and methods of use. EP 2492355 A1 [Patent]

Jacobs, K., Shoemaker, C., Rudersdorf, R., \& Neill, S., 1985. Isolation and characterization of genomic and cDNA clones of human erythropoietin. Nature, 313(28), pp.806-810.

Jacobs, M.D. \& Harrison, S.C., 1998. Structure of an IкBa/NF-кB complex. Cell, 95(6), pp.749-758.

Jan, L.Y. \& Jan, Y.N., 1982. Antibodies to horseradish peroxidase as specific neuronal markers in Drosophila and in grasshopper embryos. Proceedings of the National Academy of Sciences of the United States of America, 79(8), pp.2700-2704.

Jantzie, L.L., Miller, R.H. \& Robinson, S., 2013. Erythropoietin signaling promotes oligodendrocyte development following prenatal systemic hypoxic-ischemic brain injury. Pediatric Research, 74(6), pp.658-667.

Jelkmann, W., 2002. The enigma of the metabolic fate of circulating erythropoietin (Epo) in view of the pharmacokinetics of the recombinant drugs rhEpo and NESP. European journal of haematology, 69, pp.265-74.

Jelkmann, W., 2013. Physiology and Pharmacology of Erythropoietin. Transfusion Medicine and Hemotherapy, 40(5), pp.302-309.

Johansson, A., 1957. The nervous system of the milkweed bug Oncopeltus fasciutus (Heteroptera, Lygaeidae). Trans. Amer. Entomol. SOC., 83, pp.119183.

De Jong, L.A.A., Uges, D.R.A., Franke, J.P., \& Bischoff, R., 2005. Receptor-ligand binding assays: technologies and applications. Journal of chromatography $B$, 829(1-2), pp.1-25.

Juul, S.E., Yachnis, A.T. \& Christensen, R.D., 1998. Tissue distribution of erythropoietin and erythropoietin receptor in the developing human fetus. Early human development, 52(3), pp.235-249.

Juul, S.E., Yachnis, A.T., Rojiani, A.M., \& Christensen, R. D., 1999. Immunohistochemical localization of erythropoietin and its receptor in the developing human brain. Pediatric and developmental pathology, 2, pp.148-58. 
Kako, E., Kaneko, N., Aoyama, M., Hida, H., Takebayashi, H., Ikenaka, K. et al., 2012. Subventricular zone-derived oligodendrogenesis in injured neonatal white matter in mice enhanced by a nonerythropoietic erythropoietin derivative. Stem Cells, 30(10), pp.2234-2247.

Kaneko, N., Kako, E. \& Sawamoto, K., 2013. Enhancement of ventricularsubventricular zone-derived neurogenesis and oligodendrogenesis by erythropoietin and its derivatives. Frontiers in cellular neuroscience, 7, p.235.

Kapuściński, J. \& Szer, W., 1979. Interactions of 4', 6-diamidine-2-phenylindole with synthetic polynucleotides. Nucleic Acids Research, 6(11), pp.3519-3534.

Kästner, A., Grube, S., El-Kordi, A., Stepniak, B., Friedrichs, H., Sargin, D. et al., 2012. Common variants of the genes encoding erythropoietin and its receptor modulate cognitive performance in schizophrenia. Molecular medicine, 18, pp.1029-40.

Katz, F., Moats, W. \& Jan, Y.N., 1988. A carbohydrate epitope expressed uniquely on the cell surface of Drosophila neurons is altered in the mutant nac (neurally altered carbohydrate). The EMBO journal, 7(11), pp.3471-3477.

Keren, H., Lev-Maor, G. \& Ast, G., 2010. Alternative splicing and evolution: diversification, exon definition and function. Nature Reviews Genetics, 11(5), pp.345-355.

Kim, S.E., Cho, J.Y., Kim, K.S., Lee, S.J., Lee, K.H., \& Choi, K.Y., 2004.

Drosophila PI3 kinase and Akt involved in insulin-stimulated proliferation and ERK pathway activation in Schneider cells. Cellular Signalling, 16(11), pp.1309-1317.

Kim, E., Magen, A. \& Ast, G., 2007. Different levels of alternative splicing among eukaryotes. Nucleic Acids Research, 35(1), pp.125-131.

Kittilson, J.D., Jones, E. \& Sheridan, M.A., 2011. ERK, Akt, and STAT5 are differentially activated by the two growth hormone receptor subtypes of a teleost fish (Oncorhynchus mykiss). Frontiers in Endocrinology, 2, p.30.

Klingmüller, U., Wu, H., Hsiao, J. G., Toker, A., Duckworth, B. C., Cantley, L. C., \& Lodish, H. F., 1997. Identification of a novel pathway important for proliferation and differentiation of primary erythroid progenitors. Proceedings of the National Academy of Sciences of the United States of America, 94(7), pp.3016-3021.

Knight, A.W. \& Billinton, N., 2001. Distinguishing GFP from cellular autofluorescence. Biophotonics International, 8(7), pp.42-50.

Kohlmeier, F., Maya-Mendoza, A. \& Jackson, D.A., 2013. EdU induces DNA damage response and cell death in mESC in culture. Chromosome Research, 21(1), pp.87-100. 
Koury, M.J. \& Bondurant, M.C., 1988. Maintenance by erythropoietin of viability and maturation of murine erythroid precursor cells. Journal of cellular physiology, 137(1), pp.65-74.

Kroemer, G., Galluzzi, L., Vandenabeele, P., Abrams, J., Alnemri, E. S., Baehrecke, E. H. et al., 2009. Classification of cell death: recommendations of the Nomenclature Committee on Cell Death 2009. Cell death and differentiation, 16(1), pp.3-11.

Kubala, M., Plášek, J. \& Amler, E., 2004. Fluorescence competition sssay for the assessment of ATP binding to an isolated domain of $\mathrm{Na}+, \mathrm{K}+-\mathrm{ATPa} e$. Physiological Research, 53, pp.109-113.

Kuhn, H., 1955. The Hungarian Method for the assignment problem. Naval Research Logistics Quarterly, 2, pp.83-97.

Kuhn, H., 1956. Variants of the Hungarian method for assignment problems. Naval Research Logistics Quarterly, 3, pp.253-258.

Kumari, S., MG. S., \& Mayor, S., 2010. Endocytosis unplugged: multiple ways to enter the cell. Cell Research, 20(3), pp.256-275.

Kumral, A. Gonenc, S., Acikgoz, O., Sonmez, A., Genc, K., Yilmaz, O. et al., 2005. Erythropoietin increases glutathione peroxidase enzyme activity and decreases lipid peroxidation levels in hypoxic-ischemic brain injury in neonatal rats. Biology of the neonate, 87(1), pp.15-18.

Lacombe, C. \& Mayeux, P., 1998. Biology of erythropoietin. Haematologica, 83(8), pp.724-732.

Lai, P.H., Everett, R., Wang, F. F., Arakawa, T., \& Goldwasser, E., 1986. Structural characterization of human erythropoietin. The Journal of biological chemistry, 261(7), pp.3116-21.

Lamaze, C., Dujeancourt, A., Baba, T., Lo, C. G., Benmerah, A., \& DautryVarsat, A., 2001. Interleukin 2 receptors and detergent-resistant membrane domains define a clathrin-independent endocytic pathway. Molecular Cell, 7(3), pp.661-671.

Lavista-Llanos, S., Centanin, L., Irisarri, M., Russo, D. M., Gleadle, J. M., Bocca, S. N. et al., 2002. Control of the hypoxic response in Drosophila melanogaster by the basic helix-loop-helix PAS protein similar. Molecular and cellular biology, 22(19), pp.6842-6853.

Lawson, A.E., Bao, H., Wickrema, A., Jacobs-Helber, S. M., \& Sawyer, S. T., 2000. Phosphatase inhibition promotes antiapoptotic but not proliferative signaling pathways in erythropoietin-dependent HCD57 cells. Blood, 96(6), pp.2084-92. 
Lee, D.H., Macintyre, J. P., Taylor, G. R., Wang, E., Plante, R. K., Tam, S. S. et al., 1999. Tepoxalin enhances the activity of an antioxidant, pyrrolidine dithiocarbamate, in attenuating tumor necrosis factor alpha-induced apoptosis in WEHI 164 cells. The Journal of pharmacology and experimental therapeutics, 289(3), pp.1465-1471.

Leevers, S.J., Weinkove, D., MacDougall, L. K., Hafen, E., \& Waterfield, M. D., 1996. The Drosophila phosphoinositide 3-kinase Dp110 promotes cell growth. The EMBO journal, 15(23), pp.6584-6594.

Leist, M., Ghezzi, P., Grasso, G., Bianchi, R., Villa, P., Fratelli, M. et al., 2004. Derivatives of erythropoietin that are tissue protective but not erythropoietic. Science, 305, pp.239-242.

Lewczuk, P., Hasselblatt, M., Kamrowski-Kruck, H., Heyer, A., Unzicker, C., Sirén, A. L., \& Ehrenreich, H., 2000. Survival of hippocampal neurons in culture upon hypoxia: effect of erythropoietin. Neuroreport, 11(16), pp.34858 .

Li, X. , Dong, X., Zou, C., \& Zhang, H., 2015. Endocytic pathway mediates refractoriness of insect Bactrocera dorsalis to RNA interference. Scientific reports, 5, p.8700.

Lin, F.K., Suggs, S., Lin, C. H., Browne, J. K., Smalling, R., Egrie, J. C. et al., 1985. Cloning and expression of the human erythropoietin gene. Proceedings of the National Academy of Sciences of the United States of America, 82(22), pp.7580-7584.

Liongue, C. \& Ward, A.C., 2007. Evolution of Class I cytokine receptors. BMC Evolutionary Biology, 7(1), p.120.

Liu, C., Shen, K., Liu, Z., \& Noguchi, C. T., 1997. Regulated human erythropoietin receptor expression in mouse brain. The Journal of biological chemistry, 272(51), pp.32395-32400.

Liu, J., Narasimhan, P., Yu, F., \& Chan, P. H., 2005. Neuroprotection by hypoxic preconditioning involves oxidative stress-mediated expression of hypoxiainducible factor and erythropoietin. Stroke, 36(6), pp.1264-1269.

Livnah, O., Stura, E. A., Middleton, S. A., Johnson, D. L., Jolliffe, L. K., \& Wilson, I. A., 1999. Crystallographic evidence for preformed dimers of erythropoietin receptor before ligand activation. Science, 283, pp.987-990.

Lizcano, J.M., Alrubaie, S., Kieloch, A., Deak, M., Leevers, S. J., \& Alessi, D. R., 2003. Insulin-induced Drosophila 66 kinase activation requires phosphoinositide 3-kinase and protein kinase B. The Biochemical journal, 374, pp.297-306.

Loiacono, L.A. \& Shapiro, D.S., 2010. Detection of Hypoxia at the Cellular Level. Critical Care Clinics, 26(2), pp.409-421. 
Lu, D., Mahmood, A., Qu, C., Goussev, A., Schallert, T., \& Chopp, M., 2005. Erythropoietin enhances neurogenesis and restores spatial memory in rats after traumatic brain injury. Journal of neurotrauma, 22(9), pp.1011-1017.

Lucas, P. \& Nagnan-Le Meillour, P., 1997. Primary culture of antennal cells of Mamestra brassicae: Morphology of cell types and evidence for biosynthesis of pheromone-binding proteins in vitro. Cell and Tissue Research, 289(2), pp.375-382.

Luo, L., Sun, Y.J., Yang, L., Huang, S., \& Wu, Y.J. et al., 2013. Avermectin induces $\mathrm{P}$-glycoprotein expression in $\mathrm{S} 2$ cells via the calcium/calmodulin/NF-kB pathway. Chemico-Biological Interactions, 203(2), pp.430-439.

Mabery, E.M. \& Schneider, D.S., 2010. The drosophila TNF ortholog Eiger is required in the fat body for a robust immune response. Journal of Innate Immunity, 2(4), pp.371-378.

Mahajan, K. \& Mahajan, N., 2013. PI3K-independent AKT activation in cancers: A treasure trove for novel therapeutics. Journal of cell physiology, 227(9), pp.3178-3184.

Maier, S.F., 2003. Bi-directional immune-brain communication: Implications for understanding stress, pain, and cognition. Brain, behavior, and immunity, 17(2), pp.69-85. Available at: http:/ / www.ncbi.nlm.nih.gov/pubmed/12676570 [Accessed March 15, 2016].

Manavalan, B., Basith, S., Choi, Y.M., Lee, G., \& Choi, S., 2010. Structurefunction relationship of cytoplasmic and nuclear IKB proteins: an in silico analysis. PloS one, 5(12), p.e15782.

Manning, B.D. \& Cantley, L.C., 2007. AKT/PKB Signaling: Navigating Downstream. Cell, 129(7), pp.1261-1274.

Manji, G.A. \& Friesen, P.D., 2001. Apoptosis in Motion: An apical, P35insensitive caspase mediates programmed cell death in insect cells. Journal of Biological Chemistry, 276(20), pp.16704-16710.

Marti, H.H., Wenger, R. H., Rivas, L. A., Straumann, U., Digicaylioglu, M., Henn, V. et al., 1996. Erythropoietin gene expression in human, monkey and murine brain. The European journal of neuroscience, 8(October 1995), pp.666-676.

Masuda, S., Nagao, M., Takahata, K., Konishi, Y., Gallyas, F., Tabira, T., \& Sasaki, R., 1993. Functional erythropoietin receptor of the cells with neural characteristics. Comparison with receptor properties of erythroid cells. The Journal of Biological Chemistry, 268(15), pp.11208-11216. 
Masuda, S., Okano, M., Yamagishi, K., Nagao, M., Ueda, M., \& Sasaki, R., 1994. A novel site of erythropoietin production. Oxygen-dependent production in cultured rat astrocytes. The Journal of biological chemistry, 269(30), pp.19488-19493.

Masuda, S., Chikuma, M. \& Sasaki, R., 1997. Insulin-like growth factors and insulin stimulate erythropoietin production in primary cultured astrocytes. Brain research, 746, pp.63-70.

Mattson, M.P. \& Meffert, M.K., 2006. Roles for NF-kappaB in nerve cell survival, plasticity, and disease. Cell death and differentiation, 13(5), pp.852860.

Maxwell, P.H., Pugh, C.W. \& Ratcliffe, P.J., 1993. Inducible operation of the erythropoietin 3' enhancer in multiple cell lines: evidence for a widespread oxygen-sensing mechanism. Proceedings of the National Academy of Sciences of the United States of America, 90(6), pp.2423-2427.

Mayuzumi, D., 2010. Role of receptor ubiquitination in erythropoietin receptor signaling. PhD Thesis. University of Iowa.

McMahon, H.T.H.T. \& Boucrot, E., 2011. Molecular mechanism and physiological functions of clathrin-mediated endocytosis. Nature reviews. Molecular cell biology, 12(8), pp.517-33.

McPherson, P.S., Ritter, B. \& Wendland, B., 2000. Clathrin-Mediated Endocytosis. Available at: http:/ / www.ncbi.nlm.nih.gov/books/NBK6479/ [Accessed October 15, 2015].

Miljus, N., Heibeck, S., Jarrar, M., Micke, M., Ostrowski, D., Ehrenreich, H., \& Heinrich, R., 2014. Erythropoietin-mediated protection of insect brain neurons involves JAK and STAT but not PI3K transduction pathways. Neuroscience, 258, pp.218-227.

Minakhina, S. \& Steward, R., 2006. Nuclear factor-kappa B pathways in Drosophila. Oncogene, 25(51), pp.6749-6757.

Miskowiak, K.W., Vinberg, M., Christensen, E. M., Bukh, J. D., Harmer, C. J., Ehrenreich, H., \& Kessing, L. V., 2014. Recombinant human erythropoietin for treating treatment-resistant depression: a double-blind, randomized, placebo-controlled phase 2 trial. Neuropsychopharmacology, 39(6), pp.1399408.

Moellering, D., McAndrew, J., Jo, H., \& Darley-Usmar, V. M., 1999. Effects of pyrrolidine dithiocarbamate on endothelial cells: Protection against oxidative stress. Free Radical Biology and Medicine, 26(9-10), pp.1138-1145.

Morgan, M.J. \& Liu, Z., 2011. Crosstalk of reactive oxygen species and NF-kB signaling. Cell Research, 21(1), pp.103-115. 
Morishita, E., Masuda, S., Nagao, M., Yasuda, Y., \& Sasaki, R., 1997.

Erythropoetin receptor is expressed in rat hippocampal and cerebral cortical neurons, and erythropoietin prevents in vitro glutamate-induced neuronal death. Neuroscience, 76(1), pp.105-116.

Mounir, Z., Krishnamoorthy, J. L., Wang, S., Papadopoulou, B., Campbell, S., Muller, W. J. et al., 2011. Akt determines cell fate through inhibition of the PERK-eIF2 phosphorylation pathway. Science Signaling, 4(192), p.ra62.

Mukhopadhyay, P. \& Chaudhuri, B.B., 2015. A survey of Hough Transform. Pattern Recognition, 48(3), pp.993-1010.

Nadam, J., Navarro, F., Sanchez, P., Moulin, C., Georges, B., Laglaine, A. et al., 2007. Neuroprotective effects of erythropoietin in the rat hippocampus after pilocarpine-induced status epilepticus. Neurobiology of Disease, 25(2), pp.412-426.

Nagai, A., Nakagawa, E., Choi, H. B., Hatori, K., Kobayashi, S., \& Kim, S. U., 2001. Erythropoietin and erythropoietin receptors in human CNS neurons, astrocytes, microglia, and oligodendrocytes grown in culture. Journal of neuropathology and experimental neurology, 60(4), pp.386-392.

Nagano, M., Suzuki, H., Ui-Tei, K., Sato, S., Miyake, T., \& Miyata, Y., 1998. H-7induced apoptosis in the cells of a Drosophila neuronal cell line through affecting unidentified H-7-sensitive substance(s). Neuroscience research, 31(2), pp.113-21.

Nakagawa, H. \& Opitz, O.G., 2007. Biological Aging: Methods and Protocols. In T. O. Tollefsbol, ed. Totowa, NJ: Humana Press, pp. 167-178. Available at: http:/ / dx.doi.org/10.1007/978-1-59745-361-5_13.

Nakamura, T., Ouchida, R., Kodama, T., Kawashima, T., Makino, Y., Yoshikawa, N. et al., 2002. Cytokine receptor common $\beta$ subunit-mediated STAT5 activation confers NF-kB activation in murine prob cell line Ba/F3 cells. Journal of Biological Chemistry, 277(8), pp.6254-6265.

Nakatogawa, S., Oda, Y., Kamiya, M., Kamijima, T., Aizawa, T., Clark, K. D. et al., 2009. A novel peptide mediates aggregation and migration of hemocytes from an insect. Current Biology, 19(9), pp.779-785.

Nakazawa, T., Shimura, M., Tomita, H., Akiyama, H., Yoshioka, Y., Kudou, H., \& Tamai, 2003. Intrinsic activation of PI3K/ Akt signaling pathway and its neuroprotective effect against retinal injury. Current eye research, 26(1), pp.55-63.

Nambu, J.R., Chen, W., Hubs, S., \& Crews, S. T., 1996. The Drosophila melanogaster similar bHLH-PAS gene encodes a protein related to human hypoxia-inducible factor 1a and Drosophila single-minded. Gene, 172, pp.249-254. 
Nanda, J.S. \& Lorsch, J.R., 2014. Labeling a Protein with Fluorophores Using NHS Ester Derivitization 1st ed., Elsevier Inc. Available at:

http:/ / linkinghub.elsevier.com/ retrieve/pii/B9780124200708000088.

Narayanan, R. \& Ramaswami, M., 2001. Endocytosis in Drosophila: Progress, possibilities, prognostications. Experimental Cell Research, 271(1), pp.28-35.

Nelson, D.E., 2004. Oscillations in NF-kB signaling control the dynamics of gene expression. Science, 306(5696), pp.704-708.

Neumann, D., Wikström, L., Watowich, S. S., \& Lodish, H. F., 1993. Intermediates in degradation of the erythropoietin receptor accumulate and are degraded in lysosomes. The Journal of biological chemistry, 268(18), pp.13639-13649.

Nogawa-Kosaka, N., Hirose, T., Kosaka, N., Aizawa, Y., Nagasawa, K., Uehara, N. et al., 2010. Structural and biological properties of erythropoietin in Xenopus laevis. Experimental hematology, 38(5), pp.363-72.

Nordlander, R.H. \& Edwards, J.S., 1970. Postembryonic brain development in the monarch butterfly, Danaus plexippus plexippus L. Wilhelm Roux' Archiv, pp.164947-260.

Nurmi, A., Vartiainen, N., Pihlaja, R., Goldsteins, G., Yrjänheikki, J., \& Koistinaho, J., 2004. Pyrrolidine dithiocarbamate inhibits translocation of nuclear factor kappa-B in neurons and protects against brain ischaemia with a wide therapeutic time window. Journal of Neurochemistry, 91(3), pp.755-765.

Ostrowski, D., Ehrenreich, H. \& Heinrich, R., 2011. Erythropoietin promotes survival and regeneration of insect neurons in vivo and in vitro. Neuroscience, 188, pp.95-108.

Ott, C., Martens, H., Hassouna, I., Oliveira, B., Erck, C., Zafeiriou, M.-P. et al., 2015. Widespread expression of erythropoietin receptor in brain and its induction by injury. Molecular medicine, 21, pp.803-815.

Ozaki, K. \& Leonard, W.J., 2002. Cytokine and cytokine receptor pleiotropy and redundancy. Journal of Biological Chemistry, 277(33), pp.29355-29358.

Pahl, H.L., 1999. Activators and target genes of Rel/NF-kappaB transcription factors. Oncogene, 18(49), pp.6853-6866.

Pankratova, S., Gu, B., Kiryushko, D., Korshunova, I., Køhler, L. B., Rathje, M. et al., 2012. A new agonist of the erythropoietin receptor, Epobis, induces neurite outgrowth and promotes neuronal survival. Journal of Neurochemistry, 121(6), pp.915-923. 
Panov, A.A., 1960. The structure of insect brain during successive stages of postembryonic development. 111 Optic lobes. Rev. Entomol. U.R.S.S., pp.3955-68.

Patil, U.S., Qu, H., Caruntu, D., O'Connor, C.J., Sharma, A., Cai, Y., \& Tarr, M.A., 2013. Labeling primary amine groups in peptides and proteins with $\mathrm{N}$ - hydroxysuccinimidyl ester modified Fe3 O4 @SiO2 nanoparticles containing cleavable disulfide-bond linkers. Bioconjugate Chemistry, 24(9), pp.1562-1569.

Peng, J., Zipperlen, P. \& Kubli, E., 2005. Drosophila sex-peptide stimulates female innate immune system after mating via the toll and Imd pathways. Current Biology, 15(18), pp.1690-1694.

Perrimon, N. \& Mahowald, A.P., 1986. 1(1)hopscotch, a larval-pupal zygotic lethal with a specific maternal effect on segmentation in Drosophila. Developmental Biology, 118(1), pp.28-41.

Pitman, R.M., 1988. Delayed effects of anoxia upon the electrical properties of an identified cockroach motoneurone. J.Exp.Biol., 135, pp.95-108.

Pizzi, M., Goffi, F., Boroni, F., Benarese, M., Perkins, S. E., Liouand, H. C., \& Spano, P., 2002. Opposing roles for NF-kB/Rel factors p65 and c-Rel in the modulation of neuron survival elicited by glutamate and interleukin-1 $\beta$. Journal of Biological Chemistry, 277(23), pp.20717-20723.

Pompe, S., 2013. Erythropoietin (EPO) -mediated neuroprotection in Drosophila melanogaster. MSc Thesis. Georg-August-University Göttingen.

Quick, J., Anthony, J., Driedger, P. E., \& Hospital, B. I., 1992. The structure and biological activities of the widely used protein kinase inhibitor, H7, differ depending on the commercial source. Biochemical and Biophysical Research Communications, 187(2), pp.657-663.

Rainey, R.C., 1951. Weather and the movements of locust swarms: A new hypothesis. Nature, 168(4286), pp.1057-1060. Available at: http:/ / www.nature.com/doifinder/10.1038/1681057a0.

Ransome, M.I. \& Turnley, A.M., 2007. Systemically delivered Erythropoietin transiently enhances adult hippocampal neurogenesis. Journal of neurochemistry, 102(6), pp.1953-65.

Reichmuth, C., Becker, S., Benz, M., Debel, K., Reisch, D., Heimbeck, G. et al., 1995. The sap47 gene of Drosophila melanogaster codes for a novel conserved neuronal protein associated with synaptic terminals. Molecular Brain Research, 32(1), pp.45-54.

Remy, I., Wilson, I.A. \& Michnick, S.W., 1999. Erythropoietin receptor activation by a ligand-induced conformation change. Science, 283(5404), pp.990-993. 
Richardson, H. \& Kumar, S., 2002. Death to flies: Drosophila as a model system to study programmed cell death. Journal of Immunological Methods, 265, pp.21-28.

Robinow, S. \& White, K., 1991. Characterization and spatial distribution of the ELAV protein during Drosophila melanogaster development. Journal of neurobiology, 22(5), pp.443-461.

Rodriguez, A., Oliver, H., Zou, H., Chen, P., Wang, X., \& Abrams, J. M., 1999. Dark is a Drosophila homologue of Apaf-1/CED-4 and functions in an evolutionarily conserved death pathway. Nature cell biology, 1(5), pp.272-9.

Romashkova, J. A. \& Makarov, S.S., 1999. NF-kappaB is a target of AKT in antiapoptotic PDGF signalling. Nature, 401(6748), pp.86-90.

Ronca, F., Chan, S.L. \& Yu, V.C., 1997. 1-(5-Isoquinolinesulfonyl)-2methylpiperazine induces apoptosis in human neuroblastoma cells, $\mathrm{SH}-$ SY5Y, through a p53-dependent pathway. J Biol Chem, 272(7), pp.4252-4260.

La Rosa, G., Cardali, S., Genovese, T., Conti, A., Di Paola, R., La Tore, D. et al., 2004. Inhibition of the nuclear factor- $\mathrm{kB}$ activation with pyrrolidine dithiocarbamate attenuating inflammation and oxidative stress after experimental spinal cord trauma in rats. J Neurosurg (Spine 1), 3, pp.311321.

Rowley, A. F. \& Powell, A., 2007. Invertebrate immune systems-specific, quasispecific, or nonspecific? Journal of Immunology, 179(11), pp.7209-7214.

Roy, S.G. \& Raikhel, A.S., 2012. Nutritional and hormonal regulation of the TOR effector $4 \mathrm{E}-$ binding protein $(4 \mathrm{E}-\mathrm{BP})$ in the mosquito Aedes aegypti. The FASEB Journal, 26(3), pp.1334-1342.

Ruscher, K., Freyer, D., Karsch, M., Isaev, N., Megow, D., Sawitzki, B. et al., 2002. Erythropoietin is a paracrine mediator of ischemic tolerance in the brain: evidence from an in vitro model. J Neurosci, 22(23), pp.10291-10301.

Sagisaka, A., Tanaka, H., Furukawa, S., \& Yamakawa, M., 2004.

Characterization of a homologue of the Rel/NF-kB transcription factor from a beetle, Allomyrina dichotoma. Biochimica et Biophysica Acta, 1678, pp.85-93.

Sakanaka, M., Wen, T. C., Matsuda, S., Masuda, S., Morishita, E., Nagao, M., \& Sasaki, R., 1998. In vivo evidence that erythropoietin protects neurons from ischemic damage. Proceedings of the National Academy of Sciences of the United States of America, 95(8), pp.4635-4640.

Salic, A. \& Mitchison, T.J., 2008. A chemical method for fast and sensitive detection of DNA synthesis in vivo. Proceedings of the National Academy of Sciences of the United States of America, 105(7), pp.2415-2420. 
Sanchez, P.E., Navarro, F. P., Fares, R. P., Nadam, J., Georges, B., Moulin, C. et al., 2009. Erythropoietin receptor expression is concordant with erythropoietin but not with common $\beta$ chain expression in the rat brain throughout the life span. The Journal of Comparative Neurology, 514(4), pp.403-414.

Sargin, D., Friedrichs, H., El-Kordi, A., \& Ehrenreich, H., 2010. Erythropoietin as neuroprotective and neuroregenerative treatment strategy: comprehensive overview of 12 years of preclinical and clinical research. Best practice $\mathcal{E}$ research. Clinical anaesthesiology, 24(4), pp.573-94.

Sargin, D., El-Kordi, A., Agarwal, A., Müller, M., Wojcik, S. M., Hassouna, I. et al., 2011. Expression of constitutively active erythropoietin receptor in pyramidal neurons of cortex and hippocampus boosts higher cognitive functions in mice. BMC biology, 9, p.27.

Sarnico, I., Lanzillotta, A., Boroni, F., Benarese, M., Alghisi, M., Schwaninger, M. et al., 2009. NF-kB p50/RelA and c-Rel-containing dimers: Opposite regulators of neuron vulnerability to ischaemia. Journal of Neurochemistry, 108(2), pp.475-485.

Sättler (née Hobom), M.B., Merkler, D., Maier, K., Stadelmann, C., Ehrenreich, H., Bähr, M., \& Diem, R., 2004. Neuroprotective effects and intracellular signaling pathways of erythropoietin in a rat model of multiple sclerosis. Cell Death and Differentiation, 11, pp.S181-S192.

Saumweber, T., Weyhersmüller, A., Hallermann, S., Diegelmann, S., Michels, B., Bucher, D. et al., 2011. Behavioral and synaptic plasticity are impaired upon lack of the synaptic protein SAP47. The Journal of neuroscience, 31(9), pp.3508-3518.

Sawyer, S., Krantz, S. \& Goldwasser, E., 1987. Binding and receptor-mediated endocytosis of erythropoietin in Friend virus-infected erythroid cells. Journal of Biological Chemistry, 262(12), pp.5554-5562.

Scanga, S.E., Ruel, L., Binari, R. C., Snow, B., Stambolic, V., Bouchard, D. et al., 2000. The conserved PI3'K/PTEN/Akt signaling pathway regulates both cell size and survival in Drosophila. Oncogene, 19, pp.3971-3977.

Scanlan, J.C., Grant, W. E., Hunter, D. M., \& Milner, R. J., 2001. Habitat and environmental factors influencing the control of migratory locusts (Locusta migratoria) with an entomopathogenic fungus (Metarhizium anisopliae). Ecological Modelling, 136(2-3), pp.223-236.

Schindler, C., Levy, D.E. \& Decker, T., 2007. JAK-STAT Signaling: From Interferons to Cytokines. Journal of Biological Chemistry, 282(28), pp.2005920063.

Schoenfeld, T.J. \& Gould, E., 2012. Stress, stress hormones, and adult neurogenesis. Experimental Neurology, 233(1), pp.12-21. 
Schreck, R., Meier, B., Männel, D. N., Dröge, W., \& Baeuerle, P. A., 1992. Dithiocarbamates as potent inhibitors of nuclear factor $\mathrm{kB}$ activation in intact cells. Journal of Experimental Medicine, 175(5), pp.1181-94.

Scott, C.L. \& Begley, C.G., 1999. The beta common chain $(\beta c)$ of the granulocyte macrophage-colony stimulating factor, interleukin-3 and interleukin-5 receptors. International Journal of Biochemistry and Cell Biology, 31(10), pp.1011-1015.

Semenza, G.L., Dureza, R. C., M.D., T., J.D., G., \& Antonarakis, S. E., 1990. Human erythropoietin gene expression in transgenic mice: multiple transcription initiation sites and cis-acting regulatory elements. Molecular and cellular biology, 10(3), pp.930-938.

Semenza, G.L. \& Wang, G.L., 1992. A nuclear factor induced by hypoxia via de novo protein synthesis binds to the human erythropoietin gene enhancer at a site required for transcriptional activation. Molecular and cellular biology, 12(12), pp.5447-5454.

Shingo, T., Sorokan, S. T., Shimazaki, T., \& Weiss, S., 2001. Erythropoietin regulates the in vitro and in vivo production of neuronal progenitors by mammalian forebrain neural stem cells. The Journal of Neuroscience, 21(24), pp.9733-9743.

Silva, M., Benito, A., Sanz, C., Prosper, F., Ekhterae, D., Nuñez, G., \& Fernandez-Luna, J. L., 1999. Erythropoietin can induce the expression of $\mathrm{Bcl}-\mathrm{x}(\mathrm{L})$ through Stat5 in erythropoietin-dependent progenitor cell lines. Journal of Biological Chemistry, 274(32), pp.22165-22169.

Sirén, A.-L., Fratelli, M., Brines, M., Goemans, C., Casagrande, S., Lewczuk, P. et al., 2001a. Erythropoietin prevents neuronal apoptosis after cerebral ischemia and metabolic stress. Proceedings of the National Academy of Sciences of the United States of America, 98(7), pp.4044-9.

Sirén, A.L., Knerlich, F., Poser, W., Gleiter, C. H., Bruck, W., \& Ehrenreich, H., 2001b. Erythropoietin and erythropoietin receptor in human ischemic/hypoxic brain. Acta Neuropathologica, 101(3), pp.271-276.

Skoulakis, E.M.C., Kalderon, D. \& Davis, R.L., 1993. Preferential expression in mushroom bodies of the catalytic subunit of protein kinase $A$ and its role in learning and memory. Neuron, 11(2), pp.197-208.

Socolovsky, M., Nam, H. S., Fleming, M. D., Haase, V. H., Brugnara, C., \& Lodish, H. F., 2001. Ineffective erythropoiesis in Stat5a-/-5b-/- mice due to decreased survival of early erythroblasts. Blood, 98(12), pp.3261-3273.

Soille, P., 2003. Morphological Image Analysis 2nd ed., Springer Berlin Heidelberg. Available at: http:/ / doi.org/10.1007/978-3-662-05088-0. 
Staveley, B.E., Ruel, L., Jin, J., Stambolic, V., Mastronardi, F. G., Heitzler, P. et al., 1998. Genetic analysis of protein kinase B (AKT) in Drosophila. Current biology, 8(10), pp.599-602.

Stengl, M. \& Hildebrand, J.G., 1990. Insect olfactory neurons in vitro: morphological and immunocytochemical characterization of male-specific antennal receptor cells from developing antennae of male Manduca sexta. Journal of Neuroscience, 10(3), pp.837-847.

Steward, R., 1987. Dorsal, an embryonic polarity gene in Drosophila, is homologous to the vertebrate proto-oncogene, c-rel. Science, 238(4827), pp.692-694.

Stöven, S., Ando, I., Kadalayil, L., Engström, Y., \& Hultmark, D., 2000. Activation of the Drosophila NF-kappaB factor Relish by rapid endoproteolytic cleavage. EMBO reports, 1(4), pp.347-352.

Stöven, S., Silverman, N., Junell, A., Hedengren-Olcott, M., Erturk, D., Engstrom, Y. et al., 2003. Caspase-mediated processing of the Drosophila NF-kB factor Relish. Proceedings of the National Academy of Sciences of the United States of America, 100(10), pp.5991-5996.

Strand, M.R., Hayakawa, Y. \& Clark, K.D., 2000. Plasmatocyte spreading peptide (PSP1) and growth blocking peptide (GBP) are multifunctional homologs. Journal of Insect Physiology, 46(5), pp.817-824.

Sugawa, M., Sakurai, Y., Ishikawa-Ieda, Y., Suzuki, H., \& Asou, H., 2002. Effects of erythropoietin on glial cell development; oligodendrocyte maturation and astrocyte proliferation. Neuroscience Research, 44(4), pp.391-403.

Sui, X., Krantz, S. B., You, M., \& Zhao, Z., 1998. Synergistic activation of MAP kinase (ERK1/2) by erythropoietin and stem cell factor is essential for expanded erythropoiesis. Blood, 92(4), pp.1142-1149.

Suliman, H.B., Majiwa, P. A., Feldman, B. F., Mertens, B., \& Logan-Henfrey, L., 1996. Cloning of a cDNA encoding bovine erythropoietin and analysis of its transcription in selected tissues. Gene, 171(2), pp.275-80.

Sun, B. \& Salvaterra, P.M., 1995. Characterization of nervana, a Drosophila melanogaster neuron-specific glycoprotein antigen recognized by antihorseradish peroxidase antibodies. Journal of neurochemistry, 65(1), pp.43443.

Sun, S.C. \& Faye, I., 1992. Affinity purification and characterization of CIF, an insect immunoresponsive factor with NF-kappa B-like properties.

Comparative biochemistry and physiology. B, Comparative biochemistry, 103(1), pp.225-33. 
Svoboda, O. \& Bartunek, P., 2015. Origins of the vertebrate erythro/megakaryocytic system. BioMed Research International, 2015, p.632171.

Swanson, T.L., Knittel, L. M., Coate, T. M., Farley, S. M., Snyder, M. A., \& Copenhaver, P. F., 2005. The insect homologue of the amyloid precursor protein interacts with the heterotrimeric $G$ protein Goo in an identified population of migratory neurons. Developmental Biology, 288(1), pp.160-178.

Syed, R.S., Reid, S. W., Li, C., Cheetham, J. C., Aoki, K. H., Liu, B. et al., 1998. Efficiency of signalling through cytokine receptors depends critically on receptor orientation. Nature, 395, pp.511-516.

Takeuchi, M., Tasaki, S., Shimada, M., \& Kobata, A., 1990. Role of sugar chains in the in vitro biological activity of recombinant human erythropoietin. Journal of Biological Chemistry, 265(21), pp.12127-12130.

Tanji, T. \& Ip, Y.T., 2005. Regulators of the Toll and Imd pathways in the Drosophila innate immune response. Trends in Immunology, 26(4), pp.193198.

Thermo Fisher Scientific Inc, 2016. NHS reaction scheme for chemical conjugation to a primary amine [Image]. Available at: https:/ / www.thermofisher.com/de/de/home/life-science/proteinbiology/protein-biology-learning-center/ protein-biology-resourcelibrary/pierce-protein-methods/amine-reactive-crosslinker-chemistry.html [Accessed March 10, 2016].

Towb, P., Bergmann, A. \& Wasserman, S.A., 2001. The protein kinase Pelle mediates feedback regulation in the Drosophila Toll signaling pathway. Development, 128(23), pp.4729-4736.

Tramontano, A.F., Muniyappa, R., Black, A. D., Blendea, M. C., Cohen, I., Deng, L. et al., 2003. Erythropoietin protects cardiac myocytes from hypoxiainduced apoptosis through an Akt-dependent pathway. Biochemical and Biophysical Research Communications, 308(4), pp.990-994.

Trincavelli, M., Da Pozzo, E., Ciampi, O., Cuboni, S., Daniele, S., Abbracchio, M., \& Martini, C., 2013. Regulation of Erythropoietin Receptor Activity in Endothelial Cells by Different Erythropoietin (EPO) Derivatives: An in Vitro Study. International Journal of Molecular Sciences, 14(2), pp.2258-2281.

Tsai, P.T., Ohab, J. J., Kertesz, N., Groszer, M., Matter, C., Gao, J. et al., 2006. A critical role of erythropoietin receptor in neurogenesis and post-stroke recovery. The Journal of neuroscience, 26(4), pp.1269-1274.

Tsuda, E., Kawanishi, G., Ueda, M., Masuda, S., \& Sasaki, R., 1990. The role of carbohydrate in recombinant human erythropoietin. European journal of biochemistry / FEBS, 188(2), pp.405-11. 
Tucker, E.S. \& Tolbert, L.P., 2003. Reciprocal interactions between olfactory receptor axons and olfactory nerve glia cultured from the developing moth Manduca sexta. Developmental Biology, 260(1), pp.9-30.

Um, M. \& Lodish, H.F., 2005. Antiapoptotic effects of erythropoietin in differentiated neuroblastoma SH-SY5Y cells require activation of both the STAT5 and AKT signaling pathways. Journal of Biological Chemistry, 281(9), pp.5648-5656.

Villa, P., Bigini, P., Mennini, T., Agnello, D., Laragione, T., Cagnotto, A. et al., 2003. Erythropoietin selectively attenuates cytokine production and inflammation in cerebral ischemia by targeting neuronal apoptosis. The Journal of experimental medicine, 198(6), pp.971-975.

Vlahos, C.J., Matter, W. F., Hui, K. Y., \& Brown, R. F., 1994. A specific inhibitor of phosphatidylinositol 3-kinase, 2-(4-morpholinyl)-8-phenyl-4H-1benzopyran-4-one (LY294002). The Journal of biological chemistry, 269(7), pp.5241-5248.

Walrafen, P., Verdier, F., Kadri, Z., Chrétien, S., Lacombe, C., \& Mayeux, P., 2005. Both proteasomes and lysosomes degrade the activated erythropoietin receptor. Blood, 105(2), pp.600-608.

Wang, G.L. \& Semenza, G.L., 1995. Purification and characterization of Hypoxia-inducible Factor 1. The Journal of biological chemistry, 270, pp.12301237.

Wang, L., Zhang, Z., Wang, Y., Zhang, R., \& Chopp, M., 2004. Treatment of stroke with erythropoietin enhances neurogenesis and angiogenesis and improves neurological function in rats. Stroke, 35(7), pp.1732-1737.

Wang, X.W., Tan, N. S., Ho, B., \& Ding, J. L., 2006a. Evidence for the ancient origin of the NF- $\mathrm{kB} / \mathrm{I} \mathrm{KB}$ cascade: its archaic role in pathogen infection and immunity. Proceedings of the National Academy of Sciences of the United States of America, 103(11), pp.4204-4209.

Wang, L., Zhang, Z. G., Zhang, R. L., Gregg, S. R., Hozeska-Solgot, A., LeTourneau, Y. et al., 2006b. Matrix metalloproteinase 2 (MMP2) and MMP9 secreted by erythropoietin-activated endothelial cells promote neural progenitor cell migration. The Journal of neuroscience, 26(22), pp.59966003.

Wang, E.T., Sandberg, R., Luo, S., Khrebtukova, I., Zhang, L., Mayr, C. et al., 2008. Alternative isoform regulation in human tissue transcriptomes. Nature, 456, pp.470-476.

Wang, X., Fang, X., Yang, P., Jiang, X., Jiang, F., Zhao, D. et al., 2014. The locust genome provides insight into swarm formation and long-distance flight. Nature Communications, 5, p.3957. 
Ward, R.J. \& Milligan, G., 2014. Structural and biophysical characterisation of G protein-coupled receptor ligand binding using resonance energy transfer and fluorescent labelling techniques. Biochimica et biophysica acta, 1838, pp.3-14.

Watanabe, S., Tada, M., Aizawa, T., Yoshida, M., Sugaya, T., Taguchi, M. et al., 2006. N-terminal mutational analysis of the interaction between growthblocking peptide (GBP) and receptor of insect immune cells. Protein Pept Lett, 13(8), pp.815-22.

Watanabe, S., Liu, Q., Davis, M. W., Hollopeter, G., Thomas, N., Jorgensen, N. B., \& Jorgensen, E. M., 2013a. Ultrafast endocytosis at Caenorhabditis elegans neuromuscular junctions. eLife, 2, p.e00723. Available at: http:/ / elifesciences.org/lookup/doi/10.7554/eLife.00723.

Watanabe, S., Rost, B.R., Camacho-Pérez, M., Davis, M. W., Söhl-Kielczynski, B., Rosenmund, C., \& Jorgensen, E. M., 2013b. Ultrafast endocytosis at mouse hippocampal synapses. Nature, 504(7479), pp.242-247.

Waterhouse, R.M., Kriventseva, E. V, Meister, S., Xi, Z., Alvarez, S., Bartholomay, L. C. et al., 2007. Evolutionary dynamics of immune-related genes and pathways in disease-vector mosquitoes. Science, 316(5832), pp.1738-1743.

Weber, A.N.R., Tauszig-Delamasure, S., Hoffmann, J. A., Lelièvre, E., Gascan, H., Ray, K. P. et al., 2003. Binding of the Drosophila cytokine Spätzle to Toll is direct and establishes signaling. Nature immunology, 4(8), pp.794-800.

Wen, D., Boissel, J. P., Tracy, T. E., Gruninger, R. H., Mulcahy, L. S., Czelusniak, J., 1993. Erythropoietin structure-function relationships: high degree of sequence homology among mammals. Blood, 82(5), pp.1507-16.

Wen, T.C., Sadamoto, Y., Tanaka, J., Zhu, P. X., Nakata, K., Yong-Jie, M. et al., 2002. Erythropoietin protects neurons against chemical hypoxia and cerebral ischemic injury by up-regulating Bcl-xL expression. Journal of Neuroscience Research, 67(6), pp.795-803.

Wienken, C.J., Baaske, P., Rothbauer, U., Braun, D., \& Duhr, S., 2010. Proteinbinding assays in biological liquids using microscale thermophoresis. Nature Communications, 1(7), p.100.

Witthuhn, B.A., Quelle, F. W., Silvennoinen, O., Yi, T., Tang, B., Miura, O., \& Ihle, J. N., 1993. JAK2 associates with the erythropoietin receptor and is tyrosine phosphorylated and activated following stimulation with erythropoietin. Cell, 74(2), pp.227-36.

Wu, H., Liu, X., Jaenisch, R., \& Lodish, H. F., 1995. Generation of committed erythroid BFU-E and CFU-E progenitors does not require erythropoietin or the erythropoietin receptor. Cell, 83(1), pp.59-67. 
Wu, Y., Shang, Y., Sun, S. \& Liu, R., 2007a. Antioxidant effect of erythropoietin on 1-methyl-4-phenylpyridinium-induced neurotoxicity in PC12 cells. European journal of pharmacology, 564(1-3), pp.47-56.

Wu, Y., Shang, Y., Sun, S., Liang, H., \& Liu, R., 2007b. Erythropoietin prevents PC12 cells from 1-methyl-4-phenylpyridinium ion-induced apoptosis via the Akt/GSK-3beta/caspase-3 mediated signaling pathway. Apoptosis, 12(8), pp.1365-75.

Wüstenberg, T., Begemann, M., Bartels, C., Gefeller, O., Stawicki, S., HinzeSelch, D. et al., 2011. Recombinant human erythropoietin delays loss of gray matter in chronic schizophrenia. Molecular psychiatry, 16(1), pp.26-36, 1.

Wyder, S., Kriventseva, E. V, Schröder, R., Kadowaki, T., \& Zdobnov, E. M., 2007. Quantification of ortholog losses in insects and vertebrates. Genome biology, 8(11), p.R242.

Xie, Y. \& Ji, Q., 2002. A new efficient ellipse detection method. In Object recognition supported by user interaction for service robots. IEEE Comput. Soc, pp.957-960. Available at: http:/ / doi.org/10.1109/ICPR.2002.1048464.

Xiong, W.C., Okano, H. O., Patel, N. H., Blendy, J. A., \& Montell, C., 1994. Repo encodes a Glial-Specific Homeo Domain Protein Required in the Drosophila nervous system. Genes \& Development, pp.981-994.

Yamaguchi, Y. \& Pasquale, E.B., 2004. Eph receptors in the adult brain. Current Opinion in Neurobiology, 14(3), pp.288-296.

Yamaguchi, K., Matsumoto, H., Ochiai, M., Tsuzuki, S., \& Hayakawa, Y., 2012. Enhanced expression of stress-responsive cytokine-like gene retards insect larval growth. Insect Biochemistry and Molecular Biology, 42(3), pp.183-192.

Yamaji, R., Okada, T., Moriya, M., Naito, M., Tsuruo, T., Miyatake, K., \& Nakano, Y, 1996. Brain capillary endothelial cells express two forms of erythropoietin receptor mRNA. European journal of biochemistry / FEBS, 239(2), pp.494-500.

Yamaoka, K., Saharinen, P., Pesu, M., Et, V., Iii, H., Silvennoinen, O., \& Shea, J. J. O., 2004. The Janus kinases (Jaks). Genome biology, 5, p.253.

Yan, R., Small, S., Desplan, C., Dearolf, C. R., \& Darnell, J. E., 1996. Identification of a Stat gene that functions in Drosophila development. Cell, 84(3), pp.421-430.

Yoshimura, A., D'Andrea, A.D. \& Lodish, H.F., 1990. Friend spleen focus forming virus glycoprotein gp55 interacts with the erythropoietin receptor in the endoplasmic reticulum and affects receptor metabolism. Proceedings of the National Academy of Sciences of the United States of America, 87(11), pp.4139-43. 
Youssoufian, H., Longmore, G., Neumann, D., Yoshimura, A., \& Lodish, H. F., 1993. Structure, function, and activation of the erythropoietin receptor. Blood, 81(9), pp.2223-2236.

Zhang, Y., Chopp, M., Mahmood, A., Meng, Y., Qu, C., \& Xiong, Y., 2012. Impact of inhibition of erythropoietin treatment-mediated neurogenesis in the dentate gyrus of the hippocampus on restoration of spatial learning after traumatic brain injury. Experimental Neurology, 235(1), pp.336-344.

Zhao, W., Kitidis, C., Fleming, M. D., Lodish, H. F., \& Ghaffari, S., 2006. Erythropoietin stimulates phosphorylation and activation of GATA-1 via the PI3-kinase/AKT signaling pathway. Blood, 107(3), pp.907-915.

Zhao, X., Coptis, V. \& Farris, S.M., 2008. Metamorphosis and adult development of the mushroom bodies of the red flour beetle, Tribolium castaneum. Developmental Neurobiology, 68(13), pp.1487-1502.

Zhao, H.W. \& Haddad, G.G., 2011. Review: Hypoxic and oxidative stress resistance in Drosophila melanogaster. Placenta, 32(Suppl 2), pp.1-10.

Zhao, H., Halicka, H. D., Li, J., Biela, E., Berniak, K., Dobrucki, J., \& Darzynkiewicz, Z., 2013. DNA damage signaling, impairment of cell cycle progression, and apoptosis triggered by 5-ethynyl-2'-deoxyuridine incorporated into DNA. Cytometry Part A, 83(11), pp.979-988. 


\section{LIST OF ABBREVIATIONS}

\begin{tabular}{|c|c|}
\hline$\%$ & percent \\
\hline a & alpha \\
\hline$\beta c$ & beta common \\
\hline$\varepsilon$ & molar extinction coefficient \\
\hline A & absorbance \\
\hline A260 & absorbance at 260 nanometer \\
\hline A280 & absorbance at 280 nanometer \\
\hline $\mathrm{A}-\mathrm{T}$ & adenine-thymine \\
\hline ATP & asenosine triphosphate \\
\hline AF488 & Alexa flour 488 \\
\hline AF633 & Alexa fluor 633 \\
\hline Akt & protein kinase B (a serine/threonine-specific protein kinase) \\
\hline $\mathrm{B}$ & path length \\
\hline bad & B-cell lymphoma 2-associated death promoter \\
\hline bcl-xL & B-cell lymphoma-extra large \\
\hline BFU-E & burst forming unit-ery throid \\
\hline BrdU & bromodeoxyuridine \\
\hline BSA & bovine serum albumin \\
\hline C & concentration \\
\hline${ }^{\circ} \mathrm{C}$ & Celsius \\
\hline cAMP & cyclic adenosine monophosphate \\
\hline cEpo & carbamylated erythropoietin \\
\hline CF & correction factor \\
\hline CFU-E & colony forming unit-erythroid \\
\hline cGMP & cyclic guanosine monophosphate \\
\hline $\mathrm{CHD}$ & cytokine receptor homology domain \\
\hline CNS & central nervous system \\
\hline ConA & concanavalin A \\
\hline CRLF3 & cytokine receptor like factor 3 \\
\hline $\mathrm{CuSO}_{4}$ & copper (II) sulfate \\
\hline Cy2 & cyanine 2 \\
\hline Cy3 & cyanine 3 \\
\hline Cy5 & cyanine 5 \\
\hline$D$. & Drosophila \\
\hline $\mathrm{dAkt}$ & Drosophila Akt \\
\hline DABCO & 1,4-diazobicyclo[2.2.2]octane \\
\hline DAPI & 4'-6-diamino-2-phenylindole \\
\hline $\mathrm{DCO}$ & catalytic subunit of Drosophila melanogaster protein kinase A \\
\hline DIC & differential interference contrast \\
\hline DMSO & dimethyl sulfoxide \\
\hline DNA & deoxyribonucleic acid \\
\hline dPI3K & Drosophila phosphatidylinositol-3-kinase \\
\hline dTOR & Drosophila target of rapamycin \\
\hline
\end{tabular}




\begin{tabular}{|c|c|}
\hline e.g. & exempli gratia (for example) \\
\hline ECL & enhanced chemiluminescence \\
\hline EdU & 5-ethynyl-2'-deoxyuridine \\
\hline EDTA & ethylenediaminetetraacetic acid \\
\hline EGF & epidermal growth factor \\
\hline ELAV & embryonic lethal abnormal visual system \\
\hline Epo & erythropoietin \\
\hline EpoR & erythropoietin receptor \\
\hline EV3 & erythropoietin human splice variant missing exon 3 \\
\hline FBS & fetal bovine serum \\
\hline FBSG & fetal bovine serum gold \\
\hline Fig. & Figure \\
\hline FM1-43 & N-(3-Triethylammoniumpropyl)-4-(4-(Dibutylamino) \\
\hline & Styryl) Pyridinium Dibromide \\
\hline g & gravity \\
\hline GBP & growth blocking peptide \\
\hline Gly & glycine \\
\hline GM & gentamicin \\
\hline Grace's & Grace's Insect Medium \\
\hline gp130 & glycoprotein 130 \\
\hline $\mathrm{h}$ & hour \\
\hline $\mathrm{H}-7$ & 1-(5 isoquinolinesulfonyl)-2-methylpiperazine \\
\hline $\mathrm{H}_{2} \mathrm{O}$ & water \\
\hline HBBS & Hank`s balanced salt solution \\
\hline $\mathrm{HCl}$ & hydrochloric acid \\
\hline $\mathrm{HeNe}$ & helium neon \\
\hline HIF & hypoxia-inducible factor \\
\hline HRE & hypoxia-responsive elements \\
\hline HRP & horseradish peroxidase \\
\hline $\mathrm{I} \kappa \mathrm{B}$ & inhibitor of карра B protein \\
\hline IKK & inhibitor of карра B protein kinase \\
\hline JAK & Janus kinase \\
\hline $\mathrm{KCl}$ & potassium chloride \\
\hline $\mathrm{KH}_{2} \mathrm{PO} 4$ & potassium dihydrogen phosphate \\
\hline $\mathrm{kDa}$ & kilodalton \\
\hline$L$. & Locusta \\
\hline L15 & Leibovitz medium \\
\hline LIF & leukemia inhibitory factor \\
\hline Lys & lysine \\
\hline M & mole \\
\hline M. & Manduca \\
\hline MAPK & mitogen-activated protein kinase \\
\hline $\min$ & minute(s) \\
\hline $\mathrm{ml}$ & milliliter \\
\hline $\mathrm{mm}$ & millimeter \\
\hline $\mathrm{mM}$ & millimolar \\
\hline Mod. & model \\
\hline
\end{tabular}




\begin{tabular}{|c|c|}
\hline mRNA & messenger ribonucleic acid \\
\hline mTOR & mammalian target of rapamycin \\
\hline mTORC2 & mammalian target of rapamycin complex 2 \\
\hline $\mathrm{N}_{2}$ & molecular nitrogen \\
\hline $\mathrm{NaCl}$ & sodium chloride \\
\hline NADPH & nicotinamid adenine dinucleotide phosphate \\
\hline $\mathrm{Na} 2 \mathrm{HPO} 4$ & disodium hydrogen phosphate \\
\hline $\mathrm{NaH} 2 \mathrm{PO} 4$ & monosodium phosphate \\
\hline NFkB & $\begin{array}{l}\text { nuclear factor kappa-light-chain-enhancer of activated B } \\
\text { cells }\end{array}$ \\
\hline NGS & normal goat serum \\
\hline NHS & N-hydroxysuccimide \\
\hline $\mathrm{nm}$ & nanometer \\
\hline NSC & neural stem cells \\
\hline $\mathrm{O}_{2}$ & molecular oxygen \\
\hline $\mathrm{OL}$ & optic lobe \\
\hline OSMR & oncostatin $\mathrm{M}$ receptor \\
\hline$P$ & expectation value \\
\hline PBS & phosphate buffered saline \\
\hline PBS-T & tris buffered saline with Triton-X-100 \\
\hline Phe & phenylalanine \\
\hline PI3K & phosphatidylinositol-3-kinase \\
\hline $\mathrm{PH}$ & pleckstrin-homology \\
\hline RePo & reversed polarity \\
\hline $\mathrm{RH}$ & Rel homology \\
\hline rhEpo & recombinant human erythropoietin \\
\hline RNA & ribonucleic acid \\
\hline RNase & ribonuclease \\
\hline ROS & reactive oxygen species \\
\hline rpm & rounds per minute \\
\hline RT & room temperature \\
\hline SAP47 & synapse associated protein of 47 kilo Dalton \\
\hline SD & standard deviation \\
\hline SDS & sodium dodecyl sulfate \\
\hline SDS-PAGE & sodium dodecyl sulfate polyacrylamide gel electrophoresis \\
\hline $\mathrm{SH} 2$ & Src homology 2 domain \\
\hline STAT & signal transducer and activator of transcription \\
\hline SVZ & subventricular zone \\
\hline$T$. & Tribolium \\
\hline TAMRA & tetramethylrhodamine \\
\hline TBS & tris buffered saline \\
\hline TBS-T & tris buffered saline with Tween \\
\hline TNF & tumor necrosis factor \\
\hline $\operatorname{Trp}$ & tryptophan \\
\hline $\mathrm{U}$ & units \\
\hline $\mathrm{V}$ & volt \\
\hline
\end{tabular}




$\begin{array}{ll}\text { Val } & \text { valine } \\ \mathrm{v} / \mathrm{v} & \text { volume per volume } \\ \mu \mathrm{g} & \text { microgram } \\ \mu \mathrm{l} & \text { microliter } \\ \mu \mathrm{m} & \text { micrometer }\end{array}$




\section{ACKNOWLEDGEMENTS}

Firstly, I would like to express my sincere gratitude to my supervisor Prof. Dr. Ralf Heinrich for putting his trust in me, for his guidance and continuous support during my thesis. I appreciate his patience and readiness to discuss any issue at any time. Also, I would like to thank him for giving me the freedom to test and try new things, regardless of the certainty of the final outcome.

I would also like to thank Prof. Dr. Dr. Hannelore Ehrenreich, my second thesis committee member, for giving me directions with constructive, insightful and inspiring discussions.

I would like to thank my other examination board members, Prof. Dr. Andreas Stumpner, Prof. Dr. Gregor Bucher, Prof. Dr. Ernst Wimmer and Prof. Dr. André Fiala for their interest in my dissertation.

I want to thank Margret Winkler, Stephanie Pauls and Silvia Gubert for their technical assistance and generous personal support. These three amazing women are without any doubt "the heart and soul“ of the lab.

In addition, a thank you to Gudrun Matthes, who kindly "decoded“many mysteries of German law, regulations and paperwork for me.

I must acknowledge as well the many undergraduate students who passed through this lab during my PhD thesis and left their personal mark. In particular, I want to thank Miriam Jarrar, Michaela Micke, Nicole Paschek, Vasko Kilian Hinze, Jan Vincent Rison and Bita Massih, who with their work contributed to the content of the thesis.

I want to thank my fellow lab mates Somdatta, Marta, Seol-hee and Paule, who shared with me not only an office, but also food, night working hours and a lot of fun. I would particularly like to single out Paule, a true friend, who gave me exactly the kind of support I needed, including babysitting.

I thank all colleagues from department for their criticism, stimulating discussions and alternative views on the project.

I thank my friends from homeland, who have consistently helped me keep perspective on what is important in life. 
I would like to thank my family for supporting me: my mother Gordana and Čikajlo, who were first to open the door of science for me, believed in me and showed me that science can be rewarding for those who are brave and patient. To my father Marko for his efforts in trying to understand the nervous system from the perspective of an electrical engineer. To my brother Mile and sister Jelena for encouraging me in many different ways. I want to thank Arian for supplying me with publications that were hard to access and for much more.

Finally, I wish to thank my son Leonid, who grew together with this dissertation and was my biggest motivation to endure on this road. 


\section{CURRICULUM VITAE}

For reasons of data protection, the Curriculum Vitae is not published in the online version. 


\section{LIST OF PUBLICATIONS AND PRESENTATIONS}

\section{Publications}

Miljus N, Massih B, Weis MA, Rison JV, Bonnas CB, Sillaber I, Ehrenreich H, Geurten BRH and Heinrich R. Neuroprotection and endocytosis: erythropoietin-binding receptors in insect nervous systems (manuscript in preparation)

Heinrich R, Günther V, Miljus N (2016). Erythropoietin-mediated neuroprotection in insects. Vitamins and Hormones (submitted)

Miljus N, Heibeck S, Jarrar M, Micke M, Ostrowski D, Ehrenreich H and Heinrich R (2014). Erythropoietin-mediated protection of insect brain neurons involves JAK and STAT but not IP3K transduction pathways. Neuroscience 258: 218-227. (DOI information: 10.1016/j.neuroscience.2013.11.020)

\section{Poster presentations at international conferences}

Miljus N, Rison JV, Massih B, Ehrenreich H, Heinrich R (2015). Erythropoietin promotes survival of insect neurons via receptor-dependent signaling. The Eleventh Göttingen Meeting of the German Neuroscience Society, Göttingen

Miljus N, Hahn N, Heinrich R (2014). Neuroprotective effects of erythropoietin in insects. The 107th Annual Meeting of the German Zoological Society, Göttingen

Miljus N, Schäfer K, Pompe S, Roesler M, Ehrenreich H, Heinrich R (2013). Erythropietin-mediated protective mechanisms in insect neurons. The Tenth Göttingen Meeting of the German Neuroscience Society, Göttingen

Miljus N, Ostrowski D, Ehrenreich H, Heinrich R (2012). Erythropoietin promotes survival and regeneration of insects neurons in vivo and in vitro. The 9th International Lübeck Conference on Pathology and Pharmacology of Erythropoietin and other Hemopoietic Growth Factors, University of Lübeck 
